\title{
ENVELOPING ALGEBRAS OF SLODOWY SLICES AND GOLDIE RANK
}

\author{
ALEXANDER PREMET
}

Dedicated to Professor T.A. Springer on the occasion of his 85th birthday

\begin{abstract}
Let $U(\mathfrak{g}, e)$ be the finite $W$-algebra associated with a nilpotent element $e$ in a complex simple Lie algebra $\mathfrak{g}=\operatorname{Lie}(G)$ and let $I$ be a primitive ideal of the enveloping algebra $U(\mathfrak{g})$ whose associated variety equals the Zariski closure of the nilpotent orbit $(\operatorname{Ad} G) e$. Then it is known that $I=\operatorname{Ann}_{U(\mathfrak{g})}\left(Q_{e} \otimes_{U(\mathfrak{g}, e)} V\right)$ for some finite dimensional irreducible $U(\mathfrak{g}, e)$-module $V$, where $Q_{e}$ stands for the generalised Gelfand-Graev $\mathfrak{g}$-module associated with $e$. The main goal of this paper is to prove that the Goldie rank of the primitive quotient $U(\mathfrak{g}) / I$ always $\operatorname{divides} \operatorname{dim} V$. For $\mathfrak{g}=\mathfrak{s l}_{n}$, we use a theorem of Joseph on Goldie fields of primitive quotients of $U(\mathfrak{g})$ to establish the equality $\operatorname{rk}(U(\mathfrak{g}) / I)=\operatorname{dim} V$. We show that this equality continues to hold for $\mathfrak{g} \neq \mathfrak{s l}_{n}$ provided that the Goldie field of $U(\mathfrak{g}) / I$ is isomorphic to a Weyl skew-field and use this result to disprove Joseph's version of the Gelfand-Kirillov conjecture formulated in the mid-1970s.
\end{abstract}

\section{Introduction}

1.1. Denote by $G$ a simple, simply connected algebraic group over $\mathbb{C}$, let $(e, h, f)$ be a nontrivial $\mathfrak{s l}_{2}$-triple in the Lie algebra $\mathfrak{g}=\operatorname{Lie}(G)$, and denote by $(\cdot, \cdot)$ the $G$-invariant bilinear form on $\mathfrak{g}$ for which $(e, f)=1$. Let $\chi \in \mathfrak{g}^{*}$ be such that $\chi(x)=$ $(e, x)$ for all $x \in \mathfrak{g}$ and write $U(\mathfrak{g}, e)$ for the enveloping algebra of the Slodowy slice $e+\operatorname{Ker}$ ad $f$ to the adjoint orbit $\mathcal{O}:=(\operatorname{Ad} G) e$; see $[27,11]$. Recall that $U(\mathfrak{g}, e)=$ $\left(\text { End }_{\mathfrak{g}} Q_{e}\right)^{\text {op }}$, where $Q_{e}$ is the generalised Gelfand-Graev $\mathfrak{g}$-module associated with the triple $(e, h, f)$. The module $Q_{e}$ is induced from a one-dimensional module $\mathbb{C}_{\chi}$ over of a nilpotent subalgebra $\mathfrak{m}$ of $\mathfrak{g}$ whose dimension equals $d(e):=\frac{1}{2} \operatorname{dim} \mathcal{O}$. The Lie subalgebra $\mathfrak{m}$ is $(\operatorname{ad} h)$-stable, all eigenvalues of ad $h$ on $\mathfrak{m}$ are negative, and $\chi$ vanishes on $[\mathfrak{m}, \mathfrak{m}]$. The action of $\mathfrak{m}$ on $\mathbb{C}_{\chi}=\mathbb{C} 1_{\chi}$ is given by $x\left(1_{\chi}\right)=\chi(x) 1_{\chi}$ for all $x \in \mathfrak{m}$. The algebra $U(\mathfrak{g}, e)$ is also known as the finite $W$-algebra associated with the pair $(\mathfrak{g}, e)$ and it shares many remarkable features with the universal enveloping algebra $U(\mathfrak{g})$. It is worth mentioning that $Q_{e}$ is free as a right module over $U(\mathfrak{g}, e)$; see [34] for detail.

From now on we identify $\mathfrak{g}$ with $\mathfrak{g}^{*}$ by using the $G$-equivariant Killing isomorphism $\mathfrak{g} \ni x \mapsto(x, \cdot) \in \mathfrak{g}^{*}$. Given a primitive ideal $I$ of $U(\mathfrak{g})$ we write $\mathcal{V} \mathcal{A}(I)$ for the associated variety of $I$. By a classical result of Lie Theory, proved by Borho-Brylinski in special cases and by Joseph in general, the variety $\mathcal{V A}(I)$ coincides with the closure of a nilpotent orbit in $\mathfrak{g}$. If $V$ is a finite dimensional irreducible $U(\mathfrak{g}, e)$-module, then it follows from Skryabin's theorem [34] that the $\mathfrak{g}$-module $Q_{e} \otimes_{U(\mathfrak{g}, e)} V$ is irreducible.

Mathematics Subject Classification (2000 revision). Primary 17B35. Secondary 17B63, $17 \mathrm{~B} 81$. 
Hence the annihilator $I_{V}:=\operatorname{Ann}_{U(\mathfrak{g})}\left(Q_{e} \otimes_{U(\mathfrak{g}, e)} V\right)$ is a primitive ideal of $U(\mathfrak{g})$. According to [28, Thm. 3.1(ii)], the variety $\mathcal{V} \mathcal{A}\left(I_{V}\right)$ coincides with Zariski closure of the orbit $\mathcal{O}$.

In [28], the author conjectured that the converse is also true, i.e. for any primitive ideal $I$ of $U(\mathfrak{g})$ with $\mathcal{V} \mathcal{A}(I)=\overline{\mathcal{O}}$ there exists a finite dimensional irreducible $U(\mathfrak{g}, e)$ module $M$ such that $I=I_{M}$. This conjecture was proved by the author in [29] under a mild technical assumption on the central character of $I$ (removed in [31]) and by Losev [19] in general. Yet another proof of the conjecture was later given by Ginzburg in [13]. Losev's proof employed his new construction of $U(\mathfrak{g}, e)$ via equivariant Fedosov quantization, whilst Ginzburg's proof was based of the notion of Harish-Chandra bimodules for quantized Slodowy slices introduced and studied in [13]. The author's proof relied almost entirely on characteristic $p$ methods.

1.2. Write $X_{\mathcal{O}}$ for the set of all primitive ideals $I$ of $U(\mathfrak{g})$ with $\mathcal{\mathcal { A }}(I)=\overline{\mathcal{O}}$ and denote by $\operatorname{Irr} U(\mathfrak{g}, e)$ the set of all isoclasses of finite dimensional irreducible $U(\mathfrak{g}, e)$ modules. For $x \in \mathfrak{g}$ set $G_{x}:=\{g \in G \mid(\operatorname{Ad} g) x=x\}$. It is well known that the group $C(e):=G_{e} \cap G_{f}$ is reductive and its finite quotient $\Gamma(e):=C(e) / C(e)^{\circ}$ identifies naturally with the component group of the centraliser $G_{e}$. From the realization of $U(\mathfrak{g}, e)$ obtained by Gan-Ginzburg [11] it is immediate that the algebraic group $C(e)$ acts on $U(\mathfrak{g}, e)$ by algebra automorphisms. Thus, we can twist the module structure $U(\mathfrak{g}, e) \times M \rightarrow M$ of any $U(\mathfrak{g}, e)$-module $M$ by an element $g \in C(e)$ to obtain a new $U(\mathfrak{g}, e)$-module, ${ }^{g} M$, with underlying vector space $M$ and the $U(\mathfrak{g}, e)$-action given by $u \cdot m=g^{-1}(u) \cdot m$ for all $u \in U(\mathfrak{g}, e)$ and $m \in M$. It turns out that if the $U(\mathfrak{g}, e)$ module $M$ is irreducible and $g \in C(e)$, then $I_{M}=I_{g_{M}}$, so that the primitive ideal $I_{M}$ depends only on the isomorphism class of $M$; see [31, 4.8], for example. We thus obtain a natural surjective map $\varphi_{e}: \operatorname{Irr} U(\mathfrak{g}, e) \rightarrow X_{\mathcal{O}}$ which assigns to an isoclass $[M] \in \operatorname{Irr} U(\mathfrak{g}, e)$ the primitive ideal $I_{M} \in \mathcal{X}_{\mathcal{O}}$, where $M$ is any representative in $[M]$. The above discussion shows that the map $\varphi_{e}$ is well defined and its fibres are stable under the action of $C(e)$.

By [28, Lemma 2.4], there is an algebra embedding $\Theta: U($ Lie $C(e)) \hookrightarrow U(\mathfrak{g}, e)$ such that the differential of the rational action of $C(e)$ on $U(\mathfrak{g}, e)$ coincides with (ad $\circ \Theta)_{\mid} \operatorname{Lie}(C(e))$. As a consequence, every two-sided ideal of $U(\mathfrak{g}, e)$ is stable under the action of the connected group $C(e)^{\circ}$. Applying this to the primitive ideals of finite codimension in $U(\mathfrak{g}, e)$ it is easy to observe that the identity component $C(e)^{\circ}$ of $C(e)$ acts trivially on $\operatorname{Irr} U(\mathfrak{g}, e)$. We thus obtain a natural action of the finite group $\Gamma(e)$ on the set $\operatorname{Irr} U(\mathfrak{g}, e)$.

1.3. Confirming another conjecture of the author (first circulated around 2007) Losev proved that each fibre of $\varphi_{e}$ is a single $\Gamma(e)$-orbit; see [20, Thm. 1.2.2]. This result shows that a generalised Gelfand-Graev model of $I \in X_{\mathcal{O}}$ is almost unique; in particular, if $I_{M}=I=I_{M^{\prime}}$ for two finite dimensional irreducible $U(\mathfrak{g}, e)$-modules $M$ and $M^{\prime}$, then necessarily $\operatorname{dim} M=\operatorname{dim} M^{\prime}$. The main goal of this paper is to relate the latter number with the Goldie rank of the primitive quotient $U(\mathfrak{g}) / I$.

Let us recall the definition of the Goldie rank of a prime Noetherian ring $\mathcal{A}$. An element of $\mathcal{A}$ is called regular if it is not a zero divisor in $\mathcal{A}$. By Goldie's theory, the multiplicative set $S$ of all regular elements of $\mathcal{A}$ satisfies the left and right Ore conditions. Therefore, it can be used to form a classical ring of fractions $Q(\mathcal{A})=$ 
$S^{-1} \mathcal{A}$; see $[9,3.6]$ for more detail. The ring $Q(\mathcal{A})$ is prime Artinian, hence isomorphic to $\operatorname{Mat}_{n}(\mathcal{D})$ for some $n \in \mathbb{N}$ and some skew-field $\mathcal{D}$. We write $n=\operatorname{rk}(\mathcal{A})$ and call $n$ the Goldie rank of $\mathcal{A}$. The division ring $\mathcal{D}$ is called the Goldie field of $\mathcal{A}$. It is well known that $\operatorname{rk}(\mathcal{A})=1$ if and only if $\mathcal{A}$ is a domain. As an important example, $U(\mathfrak{g})$ admits a classical ring of fractions which is a skew-field. It is sometimes referred to as the Lie field of $\mathfrak{g}$ and denoted by $K(\mathfrak{g})$. The $n$th Weyl algebra

$$
\mathbf{A}_{n}(\mathbb{C}):=\left\langle X_{i}, \partial / \partial X_{i} \mid 1 \leq i \leq n\right\rangle_{\mathbb{C}}
$$

is a Noetherian domain, too, and the classical rings of fractions $Q\left(\mathbb{A}_{n}(\mathbb{C})\right)$, where $n \in \mathbb{N}$, are known as Weyl skew-fields.

More generally, it follows from the Feith-Utumi theorem that the Goldie rank of $\mathcal{A}$ coincides with the maximum value of $k \in \mathbb{N}$ for which there is an $x \in \mathcal{A}$ with $x^{k}=0$ and $x^{k-1} \neq 0$ (we adopt the standard convention that $x^{0}=1$ for any $x \in \mathcal{A}$ ). This is an elegant internal characterization of Goldie rank, but it is not very useful in practice. Recall that a two-sided ideal $I$ of $U(\mathfrak{g})$ is called completely prime if $\operatorname{rk}(U(\mathfrak{g}) / I)=1$, that is, if $U(\mathfrak{g}) / I$ is a domain.

In [19], Losev proved that for every finite dimensional irreducible $U(\mathfrak{g}, e)$-module $M$ the inequality $\left.\operatorname{rk}\left(U(\mathfrak{g}) / I_{M}\right)\right) \leq \operatorname{dim} M$ holds. Our first theorem strengthens this result:

Theorem A. Let $M$ be a finite dimensional irreducible $U(\mathfrak{g}, e)$-module and let $I_{M}=$ $\operatorname{Ann}_{U(\mathfrak{g})}\left(Q_{e} \otimes_{U(\mathfrak{g}, e)} M\right)$ be the corresponding primitive ideal in $X_{\mathcal{O}}$. Then the Goldie rank of the primitive quotient $U(\mathfrak{g}) / I_{M}$ divides $\operatorname{dim} M$.

Our proof of Theorem A relies on reduction modulo $\mathfrak{P}$ in the spirit of [29] and [31, Sect. 4] and makes use of the techniques introduced in [30, Sect. 2].

Denote by $x_{\lambda}$ the set of all primitive ideals of $U(\mathfrak{g})$ with central character $\lambda$. This is a finite set partially ordered by inclusion. By an old result of Dixmier, $X_{\lambda}$ contains a unique maximal element which will be denoted by $I_{\max }(\lambda)$. It was pointed out to the author by Losev (who attributes the observation to Vogan) that if $\mathfrak{g}=\mathfrak{s p}_{2 n}$ and $e$ is a nilpotent element of $\mathfrak{g}$ corresponding to partition $\left(2^{n}\right)$ of $2 n$, then $\mathcal{V} \mathcal{A}\left(I_{\max }(\rho / 2)\right)=$ $\overline{\mathcal{O}}$ and the multiplicity of $\overline{\mathcal{O}}$ in $\operatorname{gr}\left(U(\mathfrak{g}) / I_{\max }(\rho / 2)\right)$ equals $2^{n-1}$ (this follows from computations made by McGovern in [23] and also from [24, Thm. 5.14(c)]). Here $\rho$ is the half-sum of positive roots and $\mathcal{O}$ is the adjoint $G$-orbit of $e$. On the other hand, $I_{\max }(\rho / 2)=I_{M}$ for some finite dimensional irreducible $U(\mathfrak{g}, e)$-module $M$ and combining Theorems 1.3.1(2) and 1.2.2 of [20] one derives that the multiplicity of $\overline{\mathcal{O}}$ in $\operatorname{gr}\left(U(\mathfrak{g}) / I_{\max }(\rho / 2)\right)$ equals $[\Gamma(e): \Gamma(e, M)](\operatorname{dim} M)^{2}$, where $\Gamma(e, M)$ stands for the stabiliser of the isoclass of $M$ in $\Gamma(e)$. Since the primitive ideal $I_{\max }(\rho / 2)$ is completely prime by $[24$, Thm. 6.15] and $\Gamma(e) \cong \mathbb{Z} / 2 \mathbb{Z}$, we see that the integer $q_{M}:=(\operatorname{dim} M) / \operatorname{rk}\left(U(\mathfrak{g}) / I_{M}\right)$ does not always divide the order of $\Gamma(e)$. In particular, it can happen (outside type A) that $\operatorname{dim} M>\operatorname{rk}\left(U(\mathfrak{g}) / I_{M}\right)$. In the present case, this inequality can be obtained more directly by showing that the largest commutative quotient $U(\mathfrak{g}, e)^{\mathrm{ab}}$ of $U(\mathfrak{g}, e)$ is a polynomial algebra in one variable generated by the image of a Casimir element of $U(\mathfrak{g})$ and by using the fact that the completely prime primitive ideal $I_{\max }(\rho / 2)$ is not induced; see Remark 4.3 for detail.

It is becoming clear now that the dimensions of irreducible $U(\mathfrak{g}, e)$-modules $M$ are in general more computable than the Goldie ranks of primitive quotients $U(\mathfrak{g}) / I_{M}$. As an 
important example, in [21] Losev uses earlier work by Miličić-Soergel and Backelin to obtain a formula for multiplicities in the category $O$ for $W$-algebras $U(\mathfrak{g}, e)$ associated with nilpotent elements $e$ of standard Levi type ( $e$ is said to be of standard Levi type if it is regular in a Levi subalgebra of $\mathfrak{g}$ ). This gives a theoretical possibility to compute the dimensions of finite dimensional irreducible $U(\mathfrak{g}, e)$-modules in Kazhdan-Lusztig terms.

There are three nilpotent orbits $\mathcal{O}$ in $\mathfrak{g}$ with the property that for $e \in \mathcal{O}$ the equality $\operatorname{rk}\left(U(\mathfrak{g}) / I_{M}\right)=\operatorname{dim} M$ holds for any finite dimensional irreducible $U(\mathfrak{g}, e)$-module $M$. First, the zero orbit has this property because $U(\mathfrak{g}, 0)=U(\mathfrak{g})$ and all primitive ideals in $X_{\{0\}}$ have finite codimension in $U(\mathfrak{g})$. Second, if $e$ lies in the regular nilpotent orbit in $\mathfrak{g}$, then classical results of Kostant on Whittaker modules show that that the algebra $U(\mathfrak{g}, e)$ is isomorphic to the centre of $U(\mathfrak{g})$ and $\operatorname{rk}\left(U(\mathfrak{g}) / I_{M}\right)=\operatorname{dim} M=1$ for any irreducible $U(\mathfrak{g}, e)$-module $M$; see [18]. Third, the minimal nonzero nilpotent orbit of $\mathfrak{g}$ enjoys the above property by $[28$, Thm. $1.2(\mathrm{v})]$. Our second theorem indicates that the same could be true for many (but not all!) nilpotent orbits in finite dimensional simple Lie algebras.

Let $\mathcal{D}_{M}$ stand for the Goldie field of the primitive quotient $U(\mathfrak{g}) / I_{M}$. When $\mathfrak{g}=\mathfrak{s l}_{n}$, Joseph proved that $\mathcal{D}_{M}$ is isomorphic to a Weyl skew-field, more precisely, to the Goldie field of the Weyl algebra $\mathbf{A}_{d(e)}(\mathbb{C})$; see [17, Thm. 10.3].

Theorem B. If $\mathcal{D}_{M}$ is isomorphic to the Goldie field of $\mathbf{A}_{d(e)}(\mathbb{C})$, then $\operatorname{rk}\left(U(\mathfrak{g}) / I_{M}\right)=$ $\operatorname{dim} M$ for any finite dimensional irreducible $U(\mathfrak{g}, e)$-module $M$.

Combining Theorem B with the result of Joseph mentioned above we see that for $\mathfrak{g}=\mathfrak{s l}_{n}$ (and for $\left.\mathfrak{g}=\mathfrak{g l}_{n}\right)$ the equality $\operatorname{rk}\left(U(\mathfrak{g}) / I_{M}\right)=\operatorname{dim} M$ holds for all nilpotent elements $e \in \mathfrak{g}$ and all finite dimensional irreducible $U(\mathfrak{g}, e)$-modules $M{ }^{1}{ }^{1}$

In view of our earlier remarks Theorem $\mathrm{B}$ enables us to describe the completely prime primitive ideals $I$ of $U\left(\mathfrak{s l}_{n}\right)$ with $\mathcal{V} \mathcal{A}(I)=\overline{\mathcal{O}}$ as exactly those $I=I_{M}$ for which $M$ is a one-dimensional $U(\mathfrak{g}, e)$-module (one should also keep in mind here that in type A the component group $\Gamma(e)$ acts trivially on $\operatorname{Irr} U(\mathfrak{g}, e))$. This description differs from the classical one which is due to Møglin [25]. Møglin's classification of the completely prime primitive ideals of $U\left(\mathfrak{s l}_{n}\right)$ stems from her confirmation of a longstanding conjecture of Dixmier according to which any completely prime primitive ideal of $U(\mathfrak{g})$, for $\mathfrak{g}=\mathfrak{g l}_{n}$ or $\mathfrak{s l}_{n}$, coincides with the annihilator of a $\mathfrak{g}$-module induced from a one-dimensional representation of a parabolic subalgebra of $\mathfrak{g}$. We remark that for any nilpotent element $e \in \mathfrak{g}=\mathfrak{s l}_{n}$ a complete description of one-dimensional $U(\mathfrak{g}, e)$-modules can be deduced from $[31,3.8]$ which, in turn, relies on the BrundanKleshchev description of the finite $W$-algebras for $\mathfrak{g l}_{n}$ as truncated shifted Yangians; see [7]. ${ }^{2}$

More generally, using Theorem B and arguing as in [31, 4.9] it is straightforward to see that for $\mathfrak{g}=\mathfrak{s l}_{n}$ and any $d \in \mathbb{N}$ the set $X_{\mathcal{O}}(d):=\left\{I \in X_{\mathcal{O}} \mid \operatorname{rk}(U(\mathfrak{g}) / I)=d\right\}$ has a natural structure of a quasi-affine algebraic variety. There is some hope that in

\footnotetext{
${ }^{1}$ Very recently, Jonathan Brundan has reproved the equality $\operatorname{rk}\left(U(\mathfrak{g}) / I_{M}\right)=\operatorname{dim} M$ for $\mathfrak{g}=\mathfrak{g l}_{n}$ by a characteristic zero argument based on earlier results of Joseph and the theory of finite $W$-algebras of type A; see [6].

${ }^{2}$ Very recently, Brundan has found a new proof of Mœglin's theorem relying almost entirely on finite $W$-algebra techniques and the equality $\operatorname{rk}\left(U(\mathfrak{g}) / I_{M}\right)=\operatorname{dim} M$ for $\mathfrak{g}=\mathfrak{g l}_{n}$; see [6].
} 
the future one would be able to combine Theorem B with the main results of [8] to determine the scale factors of all Goldie rank polynomials for $\mathfrak{g}=\mathfrak{s l}_{n}{ }^{3}{ }^{3}$

At this point it should be mentioned that a conjecture of Joseph (put forward in 1976) asserts that the Goldie field of a primitive quotient of $U(\mathfrak{g})$ is always isomorphic to a Weyl skew-field; see $[16,1.2]$ and references therein. It is needless to say that Joseph's conjecture was inspired by the well known Gelfand-Kirillov conjecture (from 1966) on the structure of the Lie field $K(\mathfrak{g})$. Curiously, the latter conjecture fails for $\mathfrak{g}$ simple outside types $\mathrm{A}_{n}, \mathrm{C}_{n}$ and $\mathrm{G}_{2}$ (see [30, Thm. 1]) and remains open in types $\mathrm{C}_{n}$ and $\mathrm{G}_{2}$ (in type $\mathrm{A}$ the conjecture was proved by Gelfand and Kirillov themselves who made use of very special properties of the so-called mirabolic subalgebras of $\mathfrak{s l}_{n}$; see [12]). Joseph's conjecture is known to hold for many primitive quotients outside type A; see [16, 17] and [15, Satz 15.24]. When it holds for all primitive quotients $U(\mathfrak{g}) / I$ with $\mathcal{V A}(I)=\overline{\mathcal{O}}$ (as happens for the three orbits mentioned above), one can parametrise the completely prime primitive ideals in $X_{\mathcal{O}}$ by the points of the affine variety $\left(\operatorname{Specm} U(\mathfrak{g}, e)^{\mathrm{ab}}\right) / \Gamma(e)$.

However, our discussion after the formulation of Theorem A shows that for $\mathfrak{g}=\mathfrak{s p}_{2 n}$ with $n \geq 3$ and a nilpotent element $e \in \mathfrak{g}$ with $n$ Jordan blocks of size 2 we have that $\operatorname{dim} M>\operatorname{rk}\left(U(\mathfrak{g}) / I_{M}\right)$ for some irreducible finite dimensional $U(\mathfrak{g}, e)$-module $M$. Applying Theorem B we now deduce that for that $M$ the Goldie field $\mathcal{D}_{M}$ is not isomorphic to a Weyl skew-field. This shows that Joseph's version of the GelfandKirillov conjecture fails for $\mathfrak{g}=\mathfrak{s p}_{2 n}$ with $n \geq 3$ (it is known that the conjecture holds for all simple Lie algebras of rank 2 , but a detailed proof of this fact is missing in the literature).

Acknowledgements. The results presented here were announced in my talks at the conferences on symmetric spaces (Levico Terme, 2010), noncommutative algebras (Canterbury, 2010) and algebraic groups (Nagoya, 2010). I would like to thank Jon Brundan, Ivan Losev and Toby Stafford for their interest and many useful discussions. I would like to express my gratitude to the referee for very careful reading and thoughtful suggestions.

\section{Reducing modulo $\mathfrak{P}$ admissible forms of primitive quotients}

2.1. Let $G$ be a simple, simply connected algebraic group over $\mathbb{C}$, and $\mathfrak{g}=\operatorname{Lie}(G)$. Let $\mathfrak{h}$ be a Cartan subalgebra of $\mathfrak{g}$ and $\Phi$ the root system of $\mathfrak{g}$ relative to $\mathfrak{h}$. Choose a basis of simple roots $\Pi=\left\{\alpha_{1}, \ldots, \alpha_{\ell}\right\}$ in $\Phi$, let $\Phi^{+}$be the corresponding positive system in $\Phi$, and put $\Phi^{-}:=-\Phi^{+}$. Let $\mathfrak{g}=\mathfrak{n}^{-} \oplus \mathfrak{h} \oplus \mathfrak{n}^{+}$be the corresponding triangular decomposition of $\mathfrak{g}$ and choose a Chevalley basis $\mathcal{B}=\left\{e_{\gamma} \mid \gamma \in \Phi\right\} \cup\left\{h_{\alpha} \mid \alpha \in \Pi\right\}$ in $\mathfrak{g}$. Set $\mathcal{B}^{ \pm}:=\left\{e_{\alpha} \mid \alpha \in \Phi^{ \pm}\right\}$. Let $\mathfrak{g}_{\mathbb{Z}}$ and $U_{\mathbb{Z}}$ denote the Chevalley $\mathbb{Z}$-form of $\mathfrak{g}$ and the Kostant $\mathbb{Z}$-form of $U(\mathfrak{g})$ associated with $\mathcal{B}$. Given a $\mathbb{Z}$-module $V$ and a $\mathbb{Z}$-algebra $A$, we write $V_{A}:=V \otimes_{\mathbb{Z}} A$.

It follows from the Dynkin-Kostant theory that any nilpotent $G$-orbit in $\mathfrak{g}$ intersects with $\mathfrak{g}_{\mathbb{Z}}$. Take a nonzero nilpotent element $e \in \mathfrak{g}_{\mathbb{Z}}$ and choose $f, h \in \mathfrak{g}_{\mathbb{Q}}$ such that $(e, h, f)$ is an $\mathfrak{s l}_{2}$-triple in $\mathfrak{g}_{\mathbb{Q}}$. Denote by $(\cdot, \cdot)$ a scalar multiple of the Killing form

\footnotetext{
${ }^{3}$ This goal has now been achieved in [6] where Brundan used the equality $\operatorname{rk}\left(U(\mathfrak{g}) / I_{M}\right)=\operatorname{dim} M$ for $\mathfrak{g}=\mathfrak{g l}_{n}$ to derive exact formulae for all Goldie rank rank polynomials in type A.
} 
$\kappa$ of $\mathfrak{g}$ for which $(e, f)=1$ and define $\chi \in \mathfrak{g}^{*}$ by setting $\chi(x)=(e, x)$ for all $x \in \mathfrak{g}$. Given $x \in \mathfrak{g}$ we set $\mathcal{O}(x):=(\operatorname{Ad} G) \cdot x$ and $d(x):=\frac{1}{2} \operatorname{dim} \mathcal{O}(x)$.

Following $[29,31]$ we call a finitely generated $\mathbb{Z}$-subalgebra $A$ of $\mathbb{C}$ admissible if $\kappa(e, f) \in A^{\times}$and all bad primes of the root system of $G$ and the determinant of the Gram matrix of $(\cdot, \cdot)$ relative to a Chevalley basis of $\mathfrak{g}$ are invertible in $A$. It follows from the definition that every admissible ring $A$ is a Noetherian domain. Moreover, it is well known (and easy to see) that for every $\mathfrak{P} \in \operatorname{Specm} A$ the residue field $A / \mathfrak{P}$ is isomorphic to $\mathbb{F}_{q}$, where $q$ is a $p$-power depending on $\mathfrak{P}$. We denote by $\Pi(A)$ the set of all primes $p \in \mathbb{N}$ that occur this way. It follows from Hilbert's Nullstellensatz, for example, that the set $\Pi(A)$ contains almost all primes in $\mathbb{N}$ (see the proof of Lemma 4.4 in [31] for more detail).

Let $\mathfrak{g}(i)=\{x \in \mathfrak{g} \mid[h, x]=i x\}$. Then $\mathfrak{g}=\bigoplus_{i \in \mathbb{Z}} \mathfrak{g}(i)$, by the $\mathfrak{s l}_{2}$-theory, and all subspaces $\mathfrak{g}(i)$ are defined over $\mathbb{Q}$. Also, $e \in \mathfrak{g}(2)$ and $f \in \mathfrak{g}(-2)$. We define a (nondegenerate) skew-symmetric bilinear form $\langle\cdot, \cdot\rangle$ on $\mathfrak{g}(-1)$ by setting $\langle x, y\rangle:=$ $(e,[x, y])$ for all $x, y \in \mathfrak{g}(-1)$. There exists a basis $B=\left\{z_{1}^{\prime}, \ldots, z_{s}^{\prime}, z_{1}, \ldots, z_{s}\right\}$ of $\mathfrak{g}(-1)$ contained in $\mathfrak{g}_{\mathbb{Q}}$ and such that

$$
\left\langle z_{i}^{\prime}, z_{j}\right\rangle=\delta_{i j}, \quad\left\langle z_{i}, z_{j}\right\rangle=\left\langle z_{i}^{\prime}, z_{j}^{\prime}\right\rangle=0 \quad(1 \leq i, j \leq s) .
$$

As explained in $[29,4.1]$, after enlarging $A$, possibly, one can assume that $\mathfrak{g}_{A}=$ $\bigoplus_{i \in \mathbb{Z}} \mathfrak{g}_{A}(i)$, that each $\mathfrak{g}_{A}(i):=\mathfrak{g}_{A} \cap \mathfrak{g}(i)$ is a freely generated over $A$ by a basis of the vector space $\mathfrak{g}(i)$, and that $B$ is a free basis of the $A$-module $\mathfrak{g}_{A}(-1)$.

Put $\mathfrak{m}:=\mathfrak{g}(-1)^{0} \oplus \sum_{i \leq-2} \mathfrak{g}(i)$ where $\mathfrak{g}(-1)^{0}$ denotes the $\mathbb{C}$-span of $z_{1}^{\prime}, \ldots, z_{s}^{\prime}$. Then $\mathfrak{m}$ is a nilpotent Lie subalgebra of dimension $d(e)$ in $\mathfrak{g}$ and $\chi$ vanishes on the derived subalgebra of $\mathfrak{m}$; see [27] for more detail. It follows from our assumptions on $A$ that $\mathfrak{m}_{A}=\mathfrak{g}_{A} \cap \mathfrak{m}$ is a free $A$-module and a direct summand of $\mathfrak{g}_{A}$. More precisely, $\mathfrak{m}_{A}=\mathfrak{g}_{A}(-1)^{0} \oplus \sum_{i \leq-2} \mathfrak{g}_{A}(i)$, where $\mathfrak{g}_{A}(-1)^{0}=\mathfrak{g}_{A} \cap \mathfrak{g}(-1)=A z_{1}^{\prime} \oplus \cdots \oplus A z_{s}^{\prime}$. Enlarging $A$ further we may assume that $e, f \in \mathfrak{g}_{A}$ and that $\left[e, \mathfrak{g}_{A}(i)\right]$ and $\left[f, \mathfrak{g}_{A}(i)\right]$ are direct summands of $\mathfrak{g}_{A}(i+2)$ and $\mathfrak{g}_{A}(i-2)$, respectively. Then $\mathfrak{g}_{A}(i+2)=\left[e, \mathfrak{g}_{A}(i)\right]$ for all $i \geq 0$.

Write $\mathfrak{g}_{e}=\operatorname{Lie}\left(G_{e}\right)$ for the centraliser of $e$ in $\mathfrak{g}$. As in $[274.2,4.3]$ we choose a basis $x_{1}, \ldots, x_{r}, x_{r+1}, \ldots, x_{m}$ of the free $A$-module $\mathfrak{p}_{A}:=\bigoplus_{i \geq 0} \mathfrak{g}_{A}(i)$ such that

(a) $x_{i} \in \mathfrak{g}_{A}\left(n_{i}\right)$ for some $n_{i} \in \mathbb{Z}_{+}$;

(b) $x_{1}, \ldots, x_{r}$ is a free basis of the $A$-module $\mathfrak{g}_{A} \cap \mathfrak{g}_{e}$;

(c) $x_{r+1}, \ldots, x_{m} \in\left[f, \mathfrak{g}_{A}\right]$.

2.2. Let $Q_{e}$ be the generalised Gelfand-Graev $\mathfrak{g}$-module associated to $e$. Recall that $Q_{e}=U(\mathfrak{g}) \otimes_{U(\mathfrak{m})} \mathbb{C}_{\chi}$, where $\mathbb{C}_{\chi}=\mathbb{C} 1_{\chi}$ is a one-dimensional $\mathfrak{m}$-module such that $x \cdot 1_{\chi}=\chi(x) 1_{\chi}$ for all $x \in \mathfrak{m}$. Given $(\mathbf{a}, \mathbf{b}) \in \mathbb{Z}_{+}^{m} \times \mathbb{Z}_{+}^{s}$ we let $x^{\mathbf{a}} z^{\mathbf{b}}$ denote the monomial $x_{1}^{a_{1}} \cdots x_{m}^{a_{m}} z_{1}^{b_{1}} \cdots z_{s}^{b_{s}}$ in $U(\mathfrak{g})$. Set $Q_{e, A}:=U\left(\mathfrak{g}_{A}\right) \otimes_{U\left(\mathfrak{m}_{A}\right)} A_{\chi}$, where $A_{\chi}=A 1_{\chi}$. Note

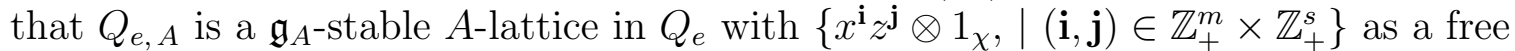
basis. Given $(\mathbf{a}, \mathbf{b}) \in \mathbb{Z}_{+}^{m} \times Z_{+}^{s}$ we set

$$
|(\mathbf{a}, \mathbf{b})|_{e}:=\sum_{i=1}^{m} a_{i}\left(n_{i}+2\right)+\sum_{i=1}^{s} b_{i} .
$$


For $\mathbf{i}=\left(i_{1}, \ldots, i_{k}\right) \in \mathbb{Z}_{+}^{k}$ set $|\mathbf{i}|:=\sum_{j=1}^{k} i_{j}$. By $[27$, Thm. 4.6], the algebra $U(\mathfrak{g}, e):=$ $\left(\operatorname{End}_{\mathfrak{g}} Q_{e}\right)^{\text {op }}$ is generated over $\mathbb{C}$ by endomorphisms $\Theta_{1}, \ldots, \Theta_{r}$ such that

$$
\Theta_{k}\left(1_{\chi}\right)=\left(x_{k}+\sum_{0<|(\mathbf{i}, \mathbf{j})|_{e} \leq n_{k}+2} \lambda_{\mathbf{i}, \mathbf{j}}^{k} x^{\mathbf{i}} z^{\mathbf{j}}\right) \otimes 1_{\chi}, \quad 1 \leq k \leq r,
$$

where $\lambda_{\mathbf{i}, \mathbf{j}}^{k} \in \mathbb{Q}$ and $\lambda_{\mathbf{i}, \mathbf{j}}^{k}=0$ if either $|(\mathbf{i}, \mathbf{j})|_{e}=n_{k}+2$ and $|\mathbf{i}|+|\mathbf{j}|=1$ or $\mathbf{i} \neq \mathbf{0}, \mathbf{j}=\mathbf{0}$, and $i_{l}=0$ for $l>r$. The monomials $\Theta_{1}^{i_{1}} \ldots \Theta_{r}^{i_{r}}$ with $\left(i_{1}, \ldots, i_{r}\right) \in \mathbb{Z}_{+}^{r}$ form a basis of the vector space $U(\mathfrak{g}, e)$.

The monomial $\Theta_{1}^{i_{1}} \cdots \Theta_{r}^{i_{r}}$ is said to have Kazhdan degree $\sum_{i=1}^{r} a_{i}\left(n_{i}+2\right)$. For $k \in \mathbb{Z}_{+}$we let $U(\mathfrak{g}, e)_{k}$ denote the $\mathbb{C}$-span of all monomials $\Theta_{1}^{i_{1}} \cdots \Theta_{r}^{i_{r}}$ of Kazhdan degree $\leq k$. The subspaces $U(\mathfrak{g}, e)_{k}, k \geq 0$, form an increasing exhaustive filtration of the algebra $U(\mathfrak{g}, e)$ called the Kazhdan filtration; see [27]. The corresponding graded algebra $\operatorname{gr} U(\mathfrak{g}, e)$ is a polynomial algebra in $\operatorname{gr} \Theta_{1}, \ldots, \operatorname{gr} \Theta_{r}$. It follows from [27, Thm. 4.6] that there exist polynomials $F_{i j} \in \mathbb{Q}\left[X_{1}, \ldots, X_{r}\right]$, where $1 \leq i<j \leq r$, such that

$$
\left[\Theta_{i}, \Theta_{j}\right]=F_{i j}\left(\Theta_{1}, \ldots, \Theta_{r}\right) \quad(1 \leq i<j \leq r) .
$$

Moreover, if $\left[x_{i}, x_{j}\right]=\sum_{k=1}^{r} \alpha_{i j}^{k} x_{k}$ in $\mathfrak{g}_{e}$, then

$$
F_{i j}\left(\Theta_{1}, \ldots, \Theta_{r}\right) \equiv \sum_{k=1}^{r} \alpha_{i j}^{k} \Theta_{k}+q_{i j}\left(\Theta_{1}, \ldots, \Theta_{r}\right) \quad\left(\bmod U(\mathfrak{g}, e)_{n_{i}+n_{j}}\right),
$$

where the initial form of $q_{i j} \in \mathbb{Q}\left[X_{1}, \ldots, X_{r}\right]$ has total degree $\geq 2$ whenever $q_{i j} \neq 0$ (as usual, by the initial form of a nonzero polynomial $f \in \mathbb{Q}\left[X_{1}, \ldots, X_{r}\right]$ we mean the nonzero component of smallest degree in the homogeneous decomposition of $f$ ). By [29, Lemma 4.1], the algebra $U(\mathfrak{g}, e)$ is generated by $\Theta_{1}, \ldots, \Theta_{r}$ subject to the relations (2). In what follows we assume that our admissible ring $A$ contains all $\lambda_{\mathbf{i}, \mathbf{j}}^{k}$ in (1) and all coefficients of the $F_{i j}$ 's in (2) (due to the above PBW theorem for $U(\mathfrak{g}, e)$ we can view the $F_{i j}$ 's as polynomials in $r=\operatorname{dim} \mathfrak{g}_{e}$ variables with coefficients in $\mathbb{Q})$.

2.3. Let $N_{\chi}$ denote the left ideal of $U(\mathfrak{g})$ generated by all $x-\chi(x)$ with $x \in \mathfrak{m}$. Then $Q_{e} \cong U(\mathfrak{g}) / N_{\chi}$ as $\mathfrak{g}$-modules. As $N_{\chi}$ is a $(U(\mathfrak{g}), U(\mathfrak{m}))$-bimodule, the fixed point space $\left(U(\mathfrak{g}) / N_{\chi}\right)^{\text {ad } \mathfrak{m}}$ carries a natural algebra structure given by $\left(x+N_{\chi}\right) \cdot\left(y+N_{\chi}\right)=$ $x y+N_{\chi}$ for all $x, y \in U(\mathfrak{g})$. Moreover, $U(\mathfrak{g}) / N_{\chi} \cong Q_{e}$ as $\mathfrak{g}$-modules via the $\mathfrak{g}$-module map sending $1+N_{\chi}$ to $1_{\chi}$, and $\left(U(\mathfrak{g}) / N_{\chi}\right)^{\text {ad } \mathfrak{m}} \cong U(\mathfrak{g}, e)$ as algebras. Any element of $U(\mathfrak{g}, e)$ is uniquely determined by its effect on the generator $1_{\chi} \in Q_{e}$ and the canonical isomorphism between $\left(U(\mathfrak{g}) / N_{\chi}\right)^{\text {ad } \mathfrak{m}}$ and $U(\mathfrak{g}, e)$ is given by $u \mapsto u\left(1_{\chi}\right)$ for all $u \in\left(U(\mathfrak{g}) / N_{\chi}\right)^{\text {ad } \mathfrak{m}}$. This isomorphism is defined over $A$. In what follows we will often identify $Q_{e}$ with $U(\mathfrak{g}) / N_{\chi}$ and $U(\mathfrak{g}, e)$ with $\left(U(\mathfrak{g}) / N_{\chi}\right)^{\text {ad } \mathfrak{m}}$.

Let $U(\mathfrak{g})=\bigcup_{j \in \mathbb{Z}} \mathrm{K}_{j} U(\mathfrak{g})$ be the Kazhdan filtration of $U(\mathfrak{g})$; see [11, 4.2]. Recall that $\mathrm{K}_{j} U(\mathfrak{g})$ is the $\mathbb{C}$-span of all products $x_{1} \cdots x_{t}$ with $x_{i} \in \mathfrak{g}\left(n_{i}\right)$ and $\sum_{i=1}^{t}\left(n_{i}+2\right) \leq$ $j$. The Kazhdan filtration on $Q_{e}$ is defined by $\mathrm{K}_{j} Q_{e}:=\pi\left(\mathrm{K}_{j} U(\mathfrak{g})\right)$ where $\pi: U(\mathfrak{g}) \rightarrow$ $U(\mathfrak{g}) / \mathcal{J}_{\chi}$ is the canonical homomorphism. It turns $Q_{e}$ into a filtered $U(\mathfrak{g})$-module. The Kazhdan grading of gr $Q_{e}$ has no negative components, and the Kazhdan filtration of $U(\mathfrak{g}, e)$ defined in 2.2 is nothing but the filtration of $U(\mathfrak{g}, e)=\left(U(\mathfrak{g}) / N_{\chi}\right)^{\text {ad } \mathfrak{m}}$ induced 
from the Kazhdan filtration of $Q_{e}$ through the embedding $\left(U(\mathfrak{g}) / N_{\chi}\right)^{\text {ad } \mathfrak{m}} \hookrightarrow Q_{e}$; see [11] for more detail.

Let $U\left(\mathfrak{g}_{A}, e\right)$ denote the $A$-span of all monomials $\Theta_{1}^{i_{1}} \ldots \Theta_{r}^{i_{r}}$ with $\left(i_{1}, \ldots, i_{r}\right) \in$ $\mathbb{Z}_{+}^{r}$. Our assumptions on $A$ guarantee that $U\left(\mathfrak{g}_{A}, e\right)$ is an $A$-subalgebra of $U(\mathfrak{g}, e)$ contained in $\left(\operatorname{End}_{\mathfrak{g}_{A}} Q_{e, A}\right)^{\mathrm{op}}$. It is immediate from the above discussion that $Q_{e, A}$ identifies with the $\mathfrak{g}_{A}$-module $U\left(\mathfrak{g}_{A}\right) / N_{\chi, A}$, where $N_{\chi, A}$ stands for the left ideal of $U\left(\mathfrak{g}_{A}\right)$ generated by all $x-\chi(x)$ with $x \in \mathfrak{m}_{A}$. Hence $U\left(\mathfrak{g}_{A}, e\right)$ embeds into the $A$ algebra $\left(U\left(\mathfrak{g}_{A}\right) / N_{\chi, A}\right)^{\text {ad } \mathfrak{m}_{A}}$. As $Q_{e, A}$ is a free $A$-module with basis consisting of all $x^{\mathbf{i}} z^{\mathbf{j}} \otimes 1_{\chi}$ with $(\mathbf{i}, \mathbf{j}) \in \mathbb{Z}_{+}^{m} \times \mathbb{Z}_{+}^{s}$ we have that

$$
U\left(\mathfrak{g}_{A}, e\right)=\left(\operatorname{End}_{\mathfrak{g}_{A}} Q_{e, A}\right)^{\mathrm{op}} \cong\left(U\left(\mathfrak{g}_{A}\right) / N_{\chi, A}\right)^{\text {ad } \mathfrak{m}_{A}} .
$$

Also, $Q_{\chi, A}$ is free as a right $U\left(\mathfrak{g}_{A}, e\right)$-module; see $[31,2.3]$ for detail.

2.4. We now pick $p \in \Pi(A)$ and denote by $\mathbb{k}$ an algebraic closure of $\mathbb{F}_{p}$. Since the form $(\cdot, \cdot)$ is $A$-valued on $\mathfrak{g}_{A}$, it induces a symmetric bilinear form on the Lie algebra $\mathfrak{g}_{\mathbb{k}} \cong \mathfrak{g}_{A} \otimes_{A} \mathbb{k}$. We use the same symbol to denote this bilinear form on $\mathfrak{g}_{\mathbb{k}}$. Let $G_{\mathbb{k}}$ be the simple, simply connected algebraic $\mathbb{k}$-group with hyperalgebra $U_{\mathbb{k}}=U_{\mathbb{Z}} \otimes_{Z} \mathbb{k}$. Note that $\mathfrak{g}_{\mathbb{k}}=\operatorname{Lie}\left(G_{\mathbb{k}}\right)$ and the form $(\cdot, \cdot)$ is $\left(\operatorname{Ad} G_{\mathbb{k}}\right)$-invariant and nondegenerate. For $x \in \mathfrak{g}_{A}$ we set $\bar{x}:=x \otimes 1$, an element of $\mathfrak{g}_{\mathbb{k}}$. To ease notation we identify $e, f$ with the nilpotent elements $\bar{e}, \bar{f} \in \mathfrak{g}_{\mathbb{k}}$ and $\chi$ with the linear function $(e, \cdot)$ on $\mathfrak{g}_{\mathbb{k}}$.

The Lie algebra $\mathfrak{g}_{\mathbb{k}}=\operatorname{Lie}\left(G_{\mathbb{k}}\right)$ carries a natural $[p]$-mapping $x \mapsto x^{[p]}$ equivariant under the adjoint action of $G_{\mathbb{k}}$. The subalgebra of $U\left(\mathfrak{g}_{\mathbb{k}}\right)$ generated by all $x^{p}-$ $x^{[p]} \in U\left(\mathfrak{g}_{\mathbb{k}}\right)$ is called the $p$-centre of $U\left(\mathfrak{g}_{\mathbb{k}}\right)$ and denoted $Z_{p}\left(\mathfrak{g}_{\mathbb{k}}\right)$ or $Z_{p}$ for short. It is immediate from the PBW theorem that $Z_{p}$ is isomorphic to a polynomial algebra in $\operatorname{dim} \mathfrak{g}$ variables and $U\left(\mathfrak{g}_{\mathfrak{k}}\right)$ is a free $Z_{p^{-}}$module of rank $p^{\operatorname{dim} \mathfrak{g}}$. For every maximal ideal $J$ of $Z_{p}$ there is a unique linear function $\eta=\eta_{J} \in \mathfrak{g}_{\mathbb{k}}^{*}$ such that

$$
J=\left\langle x^{p}-x^{[p]}-\eta(x)^{p} 1 \mid x \in \mathfrak{g}_{\mathbb{k}}\right\rangle .
$$

Since the Frobenius map of $\mathbb{k}$ is bijective, this enables us to identify the maximal spectrum $\operatorname{Specm} Z_{p}$ with $\mathfrak{g}_{\mathbb{k}}^{*}$.

Given $\xi \in \mathfrak{g}_{\mathbb{k}}^{*}$ we denote by $I_{\xi}$ the two-sided ideal of $U\left(\mathfrak{g}_{\mathbb{k}}\right)$ generated by all $x^{p}-$ $x^{[p]}-\xi(x)^{p} 1$ with $x \in \mathfrak{g}_{\mathbb{k}}$, and set $U_{\xi}\left(\mathfrak{g}_{\mathbb{k}}\right):=U\left(\mathfrak{g}_{\mathbb{k}}\right) / I_{\xi}$. The algebra $U_{\xi}\left(\mathfrak{g}_{\mathbb{k}}\right)$ is called the reduced enveloping algebra of $\mathfrak{g}_{\mathbb{k}}$ associated to $\xi$. The preceding remarks imply that $\operatorname{dim}_{\mathbb{k}} U_{\xi}\left(\mathfrak{g}_{\mathbb{k}}\right)=p^{\operatorname{dim} \mathfrak{g}}$ and $I_{\xi} \cap Z_{p}=J_{\xi}$, the maximal ideal of $Z_{p}$ associated with $\xi$. Every irreducible $\mathfrak{g}_{\mathbb{k}}$-module is a module over $U_{\xi}\left(\mathfrak{g}_{\mathbb{k}}\right)$ for a unique $\xi=\xi_{V} \in \mathfrak{g}_{\mathbb{k}}^{*}$. The linear function $\xi_{V}$ is called the p-character of $V$; see [26] for more detail. By [26], any irreducible $U_{\xi}\left(\mathfrak{g}_{\mathbb{k}}\right)$-module has dimension divisible by $p^{(\operatorname{dim} \mathfrak{g}-\operatorname{dim} \mathfrak{z} \xi) / 2}$, where $\mathfrak{z}_{\xi}=\left\{x \in \mathfrak{g}_{\mathbb{k}} \mid \xi\left(\left[x, \mathfrak{g}_{\mathbb{k}}\right]\right)=0\right\}$ is the stabiliser of $\xi$ in $\mathfrak{g}_{\mathbb{k}}$. We denote by $Z_{G_{\mathbb{k}}}(\xi)$ the coadjoint stabiliser of $\xi$ in $G_{\mathbb{k}}$.

2.5. For $i \in \mathbb{Z}$, set $\mathfrak{g}_{\mathbb{k}}(i):=\mathfrak{g}_{A}(i) \otimes_{A} \mathbb{k}$ and put $\mathfrak{m}_{\mathbb{k}}:=\mathfrak{m}_{A} \otimes_{A} \mathbb{k}$. Our assumptions on $A$ yield that the elements $\bar{x}_{1}, \ldots, \bar{x}_{r}$ form a basis of the centraliser $\left(\mathfrak{g}_{\mathrm{k}}\right)_{e}$ of $e$ in $\mathfrak{g}_{\mathrm{k}}$ and that $\mathfrak{m}_{\mathbb{k}}$ is a nilpotent subalgebra of dimension $d(e)$ in $\mathfrak{g}_{\mathbb{k}}$. Set $Q_{e, \mathbb{k}}:=U\left(\mathfrak{g}_{\mathbb{k}}\right) \otimes_{U\left(\mathfrak{m}_{\mathfrak{k}}\right)} \mathbb{k}_{\chi}$, where $\mathbb{k}_{\chi}=A_{\chi} \otimes_{A} \mathbb{k}=\mathbb{k}_{\chi}$. Clearly, $\mathbb{k}_{\chi}$ is a one-dimensional $\mathfrak{m}_{\mathbb{k}}$-module with the property that $x\left(1_{\chi}\right)=\chi(x) 1_{\chi}$ for all $x \in \mathfrak{m}_{\mathbb{k}}$. It follows from our discussion in 2.2 
and 2.3 that $Q_{e, \mathbb{k}} \cong Q_{e, A} \otimes_{A} \mathbb{k}$ as modules over $\mathfrak{g}_{\mathbb{k}}$ and $Q_{e, \mathbb{k}}$ is a free right module over the $\mathbb{k}$-algebra

$$
U\left(\mathfrak{g}_{\mathbb{k}}, e\right):=U\left(\mathfrak{g}_{A}, e\right) \otimes_{A} \mathbb{k} .
$$

Thus we may identify $U\left(\mathfrak{g}_{\mathbb{k}}, e\right)$ with a subalgebra of $\widehat{U}\left(\mathfrak{g}_{\mathbb{k}}, e\right):=\left(\operatorname{End}_{\mathfrak{g l}_{\mathbb{k}}} Q_{e, \mathbb{k}}\right)^{\text {op }}$. The algebra $U\left(\mathfrak{g}_{\mathbb{k}}, e\right)$ has $\mathbb{k}$-basis consisting of all monomials $\bar{\Theta}_{1}^{i_{1}} \cdots \bar{\Theta}_{r}^{i_{r}}$ with $\left(i_{1}, \ldots, i_{r}\right) \in$ $\mathbb{Z}_{+}^{r}$, where $\bar{\Theta}_{i}:=\Theta_{i} \otimes 1 \in U\left(\mathfrak{g}_{A}, e\right) \otimes_{A} \mathbb{k}$. Given $g \in A\left[X_{1}, \ldots, X_{n}\right]$ we write ${ }^{p} g$ for the image of $g$ in the polynomial algebra $\mathbb{k}\left[X_{1}, \ldots, X_{n}\right]=A\left[X_{1}, \ldots, X_{n}\right] \otimes_{A} \mathbb{k}$. Since all polynomials $F_{i j}$ are in $A\left[X_{1}, \ldots, X_{r}\right]$, it follows from the relations (2) that

$$
\left[\bar{\Theta}_{i}, \bar{\Theta}_{j}\right]={ }^{p} F_{i j}\left(\bar{\Theta}_{1}, \ldots, \bar{\Theta}_{r}\right) \quad(1 \leq i<j \leq r) .
$$

By [31, Lemma 2.1], the algebra $U\left(\mathfrak{g}_{\mathfrak{k}}, e\right)$ is generated by the elements $\bar{\Theta}_{1}, \ldots, \bar{\Theta}_{r}$ subject to the relations (4).

Let $\mathfrak{g}_{A}^{*}$ be the $A$-module dual to $\mathfrak{g}_{A}$ and let $\mathfrak{m}_{A}^{\perp}$ denote the set of all linear functions on $\mathfrak{g}_{A}$ vanishing on $\mathfrak{m}_{A}$. By our assumptions on $A$, this is a free $A$-submodule and a direct summand of $\mathfrak{g}_{A}^{*}$. Note that $\mathfrak{m}_{A}^{\perp} \otimes_{A} \mathbb{C}$ and $\mathfrak{m}_{A}^{\perp} \otimes_{A} \mathbb{k}$ identify naturally with with the annihilators $\mathfrak{m}^{\perp}:=\left\{f \in \mathfrak{g}^{*} \mid f(\mathfrak{m})=0\right\}$ and $\mathfrak{m}_{\mathbb{k}}^{\perp}:=\left\{f \in \mathfrak{g}_{\mathbb{k}}^{*} \mid f\left(\mathfrak{m}_{\mathbb{k}}\right)=0\right\}$, respectively.

Following [31], for $\eta \in \chi+\mathfrak{m}_{\mathbb{k}}^{\perp}$ we set $Q_{e}^{\eta}:=Q_{e, \mathbb{k}} / I_{\eta} Q_{e, \mathbb{k}}$. By construction, $Q_{e}^{\eta}$ is a $\mathfrak{g}_{\mathbb{k}}$-module with $p$-character $\eta$. Each $\mathfrak{g}_{\mathbb{k}}$-endomorphism $\bar{\Theta}_{i}$ of $Q_{e, \mathbb{k}}$ preserves $I_{\eta} Q_{e, \mathbb{k}}$, hence induces a $\mathfrak{g}_{\mathbb{k}_{\mathrm{k}}}$-endomorphism of $Q_{e}^{\eta}$ which we denote by $\theta_{i}$. We write $U_{\eta}\left(\mathfrak{g}_{\mathrm{k}}, e\right)$ for the algebra $\left(\operatorname{End}_{\mathfrak{g}_{\mathrm{k}}} Q_{e}^{\eta}\right)^{\mathrm{op}}$. Since the restriction of $\eta$ to $\mathfrak{m}_{\mathrm{k}}$ coincides with that of $\chi$, the left ideal of $U\left(\mathfrak{g}_{\mathbb{k}}\right)$ generated by all $x-\eta(x)$ with $x \in \mathfrak{m}_{\mathbb{k}}$ equals $N_{\chi, \mathbb{k}}:=N_{\chi, A} \otimes_{A} \mathbb{k}$ and $\mathbb{k}_{\chi}=\mathbb{k}_{\eta}$ as $\mathfrak{m}_{\mathbb{k}}$-modules. We denote by $N_{\eta, \chi}$ the left ideal of $U_{\eta}\left(\mathfrak{g}_{\mathbb{k}}\right)$ generated by all $x-\chi(x)$ with $x \in \mathfrak{m}_{\mathbb{k}}$. The following are proved in $[31,2.6]$ :

(a) $Q_{e}^{\eta} \cong U_{\eta}\left(\mathfrak{g}_{\mathbb{k}}\right) \otimes_{U_{\eta}\left(\mathfrak{m}_{\mathrm{k}}\right)} \mathbb{k}_{\chi}$ as $\mathfrak{g}_{\mathbb{k}}$-modules;

(b) $U_{\eta}\left(\mathfrak{g}_{\mathbb{k}}, e\right) \cong\left(U_{\eta}\left(\mathfrak{g}_{\mathbb{k}}\right) / U_{\eta}\left(\mathfrak{g}_{\mathbb{k}}\right) N_{\eta, \chi}\right)^{\text {ad } \mathfrak{m}_{\mathbb{k}}}$;

(c) $Q_{e}^{\eta}$ is a projective generator for $U_{\eta}\left(\mathfrak{g}_{\mathbb{k}}\right)$ and $U_{\eta}\left(\mathfrak{g}_{\mathbb{k}}\right) \cong \operatorname{Mat}_{p^{d(e)}}\left(U_{\eta}\left(\mathfrak{g}_{\mathbb{k}}, e\right)\right)$;

(d) the monomials $\theta_{1}^{i_{1}} \cdots \theta_{r}^{i_{r}}$ with $0 \leq i_{k} \leq p-1$ form a $\mathbb{k}$-basis of $U_{\eta}\left(\mathfrak{g}_{\mathbb{k}}, e\right)$.

Moreover, a Morita equivalence between $U_{\eta}\left(\mathfrak{g}_{\mathrm{k}}, e\right)$-mod and $U_{\eta}\left(\mathfrak{g}_{\mathrm{k}}\right)$-mod in part (b) is given explicitly by the functor that sends a finite dimensional $U_{\eta}\left(\mathfrak{g}_{\mathbb{k}}, e\right)$-module $W$ to the $U_{\eta}\left(\mathfrak{g}_{\mathbb{k}}\right)$-module $\widetilde{W}=Q_{e}^{\eta} \otimes_{U_{\eta}\left(\mathfrak{g}_{\mathbb{k}}, e\right)} W$, whilst the quasi-inverse functor from $U_{\eta}\left(\mathfrak{g}_{\mathbb{k}}\right)$-mod to $U_{\eta}\left(\mathfrak{g}_{\mathbb{k}}, e\right)$-mod sends a $U_{\eta}\left(\mathfrak{g}_{\mathbb{k}}\right)$-module $\widetilde{W}$ to its subspace

$$
W=\mathrm{Wh}_{\eta}(\widetilde{W}):=\left\{v \in \widetilde{W} \mid x \cdot v=\eta(x) v \text { for all } x \in \mathfrak{m}_{\mathbb{k}}\right\} .
$$

Recall from 2.1 the $A$-basis $\left\{x_{1}, \ldots, x_{r}, x_{r+1}, \ldots, x_{m}\right\}$ of $\mathfrak{p}_{A}$ and set

$$
X_{i}= \begin{cases}z_{i} & \text { if } 1 \leq i \leq s, \\ x_{r-s+i} & \text { if } s+1 \leq i \leq m-r+s .\end{cases}
$$

For $\mathbf{a} \in \mathbb{Z}_{+}^{d(e)}$, put $X^{\mathbf{a}}:=X_{1}^{a_{1}} \cdots X_{d(e)}^{a_{d(e)}}$ and $\bar{X}^{\mathbf{a}}:=\bar{X}_{1}^{a_{1}} \cdots \bar{X}_{d(e)}^{a_{d(e)}}$, elements of $U\left(\mathfrak{g}_{A}\right)$ and $U\left(\mathfrak{g}_{\mathbb{k}}\right)$, respectively. By [29, Lemma $\left.4.2(\mathrm{i})\right]$, the vectors $X^{\mathbf{a}} \otimes 1_{\chi}$ with $\mathbf{a} \in \mathbb{Z}^{d(e)}$ form a free basis of the right $U\left(\mathfrak{g}_{A}, e\right)$-module $Q_{e, A}$. Let $\mathfrak{a}_{\mathbb{k}}$ be the $\mathbb{k}$-span of $\bar{X}_{1}, \ldots, \bar{X}_{d(e)}$ in $\mathfrak{g}_{\mathbb{k}}$ and put $\widetilde{\mathfrak{a}}_{\mathbb{k}}:=\mathfrak{a}_{\mathbb{k}} \oplus \mathfrak{z} \chi$. Note that $\mathfrak{a}_{\mathbb{k}}=\left\{x \in \widetilde{\mathfrak{a}}_{\mathbb{k}} \mid(x, \operatorname{Ker} \operatorname{ad} f)=0\right\}$. Since $\chi$ vanishes on $\widetilde{\mathfrak{a}}_{\mathbb{k}}$, we may identify the symmetric algebra $S\left(\widetilde{\mathfrak{a}}_{\mathbb{k}}\right)$ with the coordinate 
ring $\mathbb{k}\left[\chi+\mathfrak{m}_{\mathbb{k}}^{\perp}\right]$ by setting $x(\eta):=\eta(x)$ for all $x \in \widetilde{\mathfrak{a}}_{\mathbb{k}}$ and $\eta \in \chi+\mathfrak{m}_{\mathbb{k}}^{\perp}$ and extending to $S\left(\widetilde{\mathfrak{a}}_{\mathbb{k}}\right)$ algebraically.

Given a subspace $V \subseteq \mathfrak{g}_{\mathbb{k}}$ we denote by $Z_{p}(V)$ the subalgebra of the $p$-centre $Z\left(\mathfrak{g}_{\mathbb{k}}\right)$ generated by all $x^{p}-x^{[p]}$ with $x \in V$. Clearly, $Z_{p}(V)$ is isomorphic to a polynomial algebra in $\operatorname{dim}_{\mathbb{k}} V$ variables. Let $\rho_{\mathbb{k}}$ denote the representation of $U\left(\mathfrak{g}_{\mathbb{k}}\right)$ in $\operatorname{End}_{\mathbb{k}} Q_{e, \mathbb{k}}$.

In $[31,2.7]$ we proved the following:

Theorem 2.1. The algebra $\widehat{U}\left(\mathfrak{g}_{\mathbb{k}}, e\right)$ is generated by $U\left(\mathfrak{g}_{\mathbb{k}}, e\right)$ and $\rho_{\mathbb{k}}\left(Z_{p}\right) \cong Z_{p}\left(\widetilde{\mathfrak{a}}_{\mathbb{k}}\right)$. Moreover, $\widehat{U}\left(\mathfrak{g}_{\mathbb{k}}, e\right)$ is a free $\rho_{\mathbb{k}}\left(Z_{p}\right)$-module with basis $\left\{\bar{\Theta}_{1}^{a_{1}} \cdots \bar{\Theta}_{r}^{a_{r}} \mid 0 \leq a_{i} \leq p-1\right\}$ and $\widehat{U}\left(\mathfrak{g}_{\mathbb{k}}, e\right) \cong U\left(\mathfrak{g}_{\mathbb{k}}, e\right) \otimes Z_{p}\left(\mathfrak{a}_{\mathbb{k}}\right)$ as $\mathbb{k}$-algebras.

Combining [31, Thm. 2.1(ii)] with [31, Lemma 2.2(iv)] it is straightforward to see that $Q_{e, \mathbb{k}}$ is a free right $\widehat{U}\left(\mathfrak{g}_{\mathbb{k}}, e\right)$-module with basis $\left\{\bar{X}_{1}^{a_{1}} \cdots \bar{X}_{d(e)}^{a_{d(e)}} \otimes 1_{\chi} \mid 0 \leq a_{i} \leq p-1\right\}$ and $U_{\eta}\left(\mathfrak{g}_{\mathbb{k}}, e\right) \cong \widehat{U}\left(\mathfrak{g}_{\mathbb{k}}, e\right) \otimes_{Z_{p}\left(\widetilde{\mathfrak{a}}_{\mathrm{k}}\right)} \mathbb{k}_{\eta}$ for every $\eta \in \chi+\mathfrak{m}_{\mathbb{k}}^{\perp}$. (The algebra $Z_{p}\left(\widetilde{\mathfrak{a}}_{\mathbb{k}}\right)$ acts on $\mathbb{k}_{\eta}=\mathbb{k}_{\eta}$ by the rule $\left(x^{p}-x^{[p]}\right)\left(1_{\eta}\right)=\eta(x)^{p}$ for all $x \in \widetilde{\mathfrak{a}}_{\mathbb{k}}$.)

2.6. From now on we fix a primitive ideal $\mathcal{J}$ of $U(\mathfrak{g})$ with $\mathcal{V A}(\mathcal{J})=\overline{\mathcal{O}}$. The affine variety $\mathcal{V} \mathcal{A}(\mathcal{J})$ is the zero locus in $\mathfrak{g}^{*} \cong \mathfrak{g}$ of the $(\operatorname{Ad} G)$-invariant ideal gr $\mathcal{J}$ of $S(\mathfrak{g})=$ $\operatorname{gr} U(\mathfrak{g})$. As we identify $\mathfrak{g}$ with $\mathfrak{g}^{*}$ by using the Killing isomorphism $\kappa$, our assumption on $\mathcal{J}$ simply means that the open $\left(\operatorname{Ad}^{*} G\right)$-orbit of $\mathcal{V} \mathcal{A}(\mathcal{J})$ contains $\chi$. We know from [19, Thm. 1.2.2], [31, Thm. 4.2] and [13, Thm. 4.5.2] that $\mathcal{J}=\operatorname{Ann}_{U(\mathfrak{g})}\left(Q_{e} \otimes_{U(\mathfrak{g}, e)} M\right)$ for some finite dimensional $U(\mathfrak{g}, e)$-module $M$. We choose a $\mathbb{C}$-basis basis $E=$ $\left\{m_{1}, \ldots, m_{l}\right\}$ of $M$ and denote by $\tilde{A}$ the $A$-subalgebra of $\mathbb{C}$ generated by the coefficients of the coordinate vectors of all $\Theta_{i}\left(m_{j}\right) \in M$ with respect to $E$. By construction, the $\operatorname{ring} \tilde{A}$ is admissible and the $\tilde{A}$-span of $E$ is a $U\left(\mathfrak{g}_{A}, e\right)$-stable $\tilde{A}$-lattice in $M$. Thus, after replacing $A$ by $\tilde{A}$ we may assume that the lattice $M_{A}:=A m_{1} \oplus \cdots \oplus A m_{l}$ of $M$ is $U\left(\mathfrak{g}_{A}, e\right)$-stable. We write $\tau_{A}$ for the corresponding representation of $U\left(\mathfrak{g}_{A}, e\right)$ in End $M_{A}$. Our discussion in 2.3 and 2.5 then shows that the $\mathfrak{g}$-module $\widetilde{M}:=$

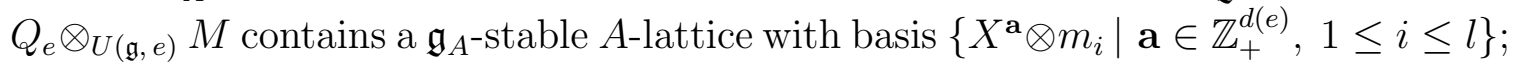
we call it $\widetilde{M}_{A}$. Note that $\widetilde{M}_{A} \cong Q_{e, A} \otimes_{U\left(\mathfrak{g}_{A}, e\right)} M_{A}$ as $\mathfrak{g}_{A}$-modules. For $p \in \Pi(A)$, the $\mathfrak{g}_{\mathbb{k}}$-module $\widetilde{M}_{\mathbb{k}}$ has $\mathbb{k}$-basis $\left\{\bar{X}^{\mathbf{a}} \otimes \bar{m}_{i} \mid \mathbf{a} \in \mathbb{Z}_{+}^{d(e)}, 1 \leq i \leq l\right\}$, where $\bar{m}_{i}=m_{i} \otimes 1$. Also, $\widetilde{M}_{\mathbb{k}} \cong Q_{e, \mathbb{k}} \otimes_{U\left(\mathfrak{g}_{\mathbb{k}}, e\right)} M_{\mathbb{k}}$ as $\mathfrak{g}_{\mathbb{k}}$-modules.

For $1 \leq i, j \leq l$ denote by $E_{i, j}$ the endomorphism of $M$ such that $E_{i, j}\left(m_{k}\right)=$ $\delta_{j, k} m_{i}$ for all $1 \leq k \leq l$. As $M$ is an irreducible $U(\mathfrak{g}, e)$-module, we may assume, after enlarging $A$ further if necessary, that all $E_{i, j}$ 's are in the image of $U\left(\mathfrak{g}_{A}, e\right)$ in End $M$. Thus we may assume that for every $p \in \Pi(A)$ the $U\left(\mathfrak{g}_{\mathbb{k}}, e\right)$-module $M_{\mathbb{k}}$ is irreducible. We mention that $U\left(\mathfrak{g}_{\mathbb{k}}, e\right)$ acts on $M_{\mathbb{k}}$ via the representation $\tau_{\mathbb{k}}=\tau_{A} \otimes 1$. By Theorem 2.1, $\widehat{U}\left(\mathfrak{g}_{\mathbb{k}}, e\right) \cong U\left(\mathfrak{g}_{\mathbb{k}}, e\right) \otimes_{\mathbb{k}} Z_{p}\left(\mathfrak{a}_{\mathbb{k}}\right)$ as $\mathbb{k}$-algebras. Therefore, for any linear function $\psi$ on $\mathfrak{a}_{\mathbb{k}}$ there is a unique representation $\widehat{\tau}_{\mathbb{k}, \psi}: \widehat{U}\left(\mathfrak{g}_{\mathbb{k}}, e\right) \rightarrow$ End $M_{\mathbb{k}}$ with $\widehat{\tau}_{\mathbb{k}, \psi}\left(x^{p}-x^{[p]}\right)=\psi(x)^{p}$ Id for all $x \in \mathfrak{a}_{\mathbb{k}}$ whose restriction to $U\left(\mathfrak{g}_{\mathbb{k}}, e\right) \hookrightarrow \widehat{U}\left(\mathfrak{g}_{\mathbb{k}}, e\right)$ coincides with $\tau_{\mathbb{k}}$. Since the representation $\widetilde{\tau}_{\mathbb{k}, \psi}$ is irreducible and $Z_{p}\left(\widetilde{\mathfrak{a}}_{\mathbb{k}}\right)$ is a central subalgebra of $\widehat{U}\left(\mathfrak{g}_{\mathrm{k}}, e\right)$, the linear function $\psi$ extends uniquely to a linear function $\Psi$ on $\widetilde{\mathfrak{a}}_{\mathbb{k}}$ such that $\widehat{\tau}_{\mathbb{k}, \psi}\left(x^{p}-x^{[p]}\right)=\Psi(x)^{p}$ Id for all $x \in \widetilde{\mathfrak{a}}_{\mathbb{k}}$. As $\mathfrak{g}_{\mathbb{k}}=\mathfrak{m}_{\mathbb{k}} \oplus \widetilde{\mathfrak{a}}_{\mathbb{k}}$, we can extend $\Psi$ to a linear function on $\mathfrak{g}_{\mathbb{k}}$ by setting $\Psi(x)=\chi(x)$ for all $x \in \mathfrak{m}_{\mathbb{k}}$. By construction, $\Psi \in \chi+\mathfrak{m}_{\mathbb{k}}^{\perp}$ and $\left.\Psi\right|_{\mathfrak{a}_{\mathrm{k}}}=\psi$. 
We now set $\widetilde{M}_{\mathbb{k}, \Psi}:=\widetilde{M}_{\mathbb{k}} / I_{\Psi} \widetilde{M}_{\mathbb{k}}$, a $\mathfrak{g}_{\mathbb{k}}$-module with $p$-character $\Psi$. The definition of $\Psi$ and our discussion in 2.5 show that

$$
\begin{aligned}
\widetilde{M}_{\mathbb{k}, \Psi} & \cong \widetilde{M}_{\mathbb{k}} \otimes_{Z_{p}\left(\mathfrak{g}_{\mathfrak{k}}\right)} \mathbb{k}_{\Psi}=\left(Q_{e, \mathbb{k}} \otimes_{U\left(\mathfrak{g}_{\mathfrak{k}}, e\right)} M_{\mathbb{k}}\right) \otimes_{Z_{p}\left(\mathfrak{m}_{\mathrm{k}}\right) \otimes Z_{p}\left(\widetilde{\mathfrak{a}}_{\mathbb{k}}\right)} \mathbb{k}_{\Psi} \\
& \cong\left(Q_{e, \mathbb{k}} \otimes_{U\left(\mathfrak{g}_{\mathfrak{k}}, e\right)} M_{\mathbb{k}}\right) \otimes_{Z_{p}\left(\widetilde{\mathfrak{a}}_{\mathfrak{k}}\right)} \mathbb{k}_{\Psi} \cong Q_{e, \mathbb{k}} \otimes_{\widehat{U}\left(\mathfrak{g}_{\mathfrak{k}}, e\right)} M_{\mathbb{k}} \cong Q_{e}^{\Psi} \otimes_{U_{\Psi}\left(\mathfrak{g}_{\mathrm{k}}, e\right)} M_{\mathbb{k}},
\end{aligned}
$$

where we view $M_{\mathbb{k}}$ as a $\widehat{U}\left(\mathfrak{g}_{\mathbb{k}}, e\right)$-module via the representation $\widehat{\tau}_{\mathbb{k}, \psi}$. This implies that under our assumptions on $A$ and $\Psi$ the $U_{\Psi}\left(\mathfrak{g}_{\mathbb{k}}\right)$-module $\widetilde{M}_{\mathbb{k}, \Psi}$ is irreducible and has dimension $l p^{d(e)}$; see 2.5 for more detail.

Remark 2.1. One can prove that the linear functions $\Psi$ constructed in this subsection form a single orbit under the action of the connected unipotent subgroup $\mathcal{M}_{\mathbb{k}}$ of $G_{\mathbb{k}}$ such that $\operatorname{Ad} \mathcal{M}_{\mathbb{k}}$ is generated by all linear operators exp ad $x$ with $x \in \mathfrak{m}_{\mathbb{k}}$. Indeed, the group $\mathcal{M}_{\mathbb{k}}$ preserves the left ideal $U\left(\mathfrak{g}_{\mathbb{k}}\right) N_{\chi, \mathbb{k}}$ and hence acts on both $Z_{p}\left(\widetilde{\mathfrak{a}}_{\mathbb{k}}\right)=$ $\rho_{\mathbb{k}}\left(Z_{p}\left(\mathfrak{g}_{\mathbb{k}}\right)\right)$ and $\widehat{U}\left(\mathfrak{g}_{\mathbb{k}}, e\right)=\left(U\left(\mathfrak{g}_{\mathbb{k}}\right) / U\left(\mathfrak{g}_{\mathbb{k}}\right) N_{\chi, \mathbb{k}}\right)$ ad $\mathfrak{m}_{\mathbb{k}}$. The rational action of $\mathcal{M}_{\mathbb{k}}$ on $Q_{e, \mathbb{k}}$ is obtained by reducing modulo $\mathfrak{P}$ the natural action on $Q_{e, A}$ of the unipotent subgroup $\mathcal{M}_{A}$ of $G$ such that $\operatorname{Ad} \mathcal{M}_{A}$ is generated by all inner automorphisms exp ad $x$ with $x \in \mathfrak{m}_{A}$. From this it follows that $U\left(\mathfrak{g}_{\mathbb{k}}, e\right) \subseteq \widehat{U}\left(\mathfrak{g}_{\mathbb{k}}, e\right)^{\mathcal{M}_{\mathfrak{k}}}$ (one should keep in mind here that $U\left(\mathfrak{g}_{\mathbb{k}}, e\right)$ is generated by $\bar{\Theta}_{1}, \ldots, \bar{\Theta}_{r}$ and $\left.p \gg 0\right)$. As we identify $S\left(\widetilde{\mathfrak{a}}_{\mathbb{k}}\right)$ with $\mathbb{k}\left[\chi+\mathfrak{m}_{\mathbb{k}}^{\perp}\right]$, we may regard the $\mathcal{M}_{\mathbb{k}}$-algebra $Z_{p}\left(\widetilde{\mathfrak{a}}_{\mathbb{k}}\right)$ as the coordinate algebra of the Frobenius twist $\left(\chi+\mathfrak{m}_{\mathbb{k}}^{\perp}\right)^{(1)} \subset\left(\mathfrak{g}_{\mathbb{k}}^{*}\right)^{(1)}$ of $\chi+\mathfrak{m}_{\mathbb{k}}^{\perp}$; see [30, 3.4] for more detail. The natural action of $\mathcal{M}_{\mathbb{k}}$ on $\left(\chi+\mathfrak{m}_{\mathbb{k}}^{\perp}\right)^{(1)}$ is a Frobenius twist of the coadjoint action of $\mathcal{M}_{\mathbb{k}}$ on $\chi+\mathfrak{m}_{\mathbb{k}}^{\perp}$. By Theorem 2.1, $\widehat{U}\left(\mathfrak{g}_{\mathrm{k}}, e\right)$ is a free $Z_{p}\left(\widetilde{\mathfrak{a}}_{\mathbb{k}}\right)$-module with basis consisting of elements from $U\left(\mathfrak{g}_{\mathbb{k}}, e\right)$. From this we deduce that $\widehat{U}\left(\mathfrak{g}_{\mathrm{k}}, e\right)^{\mathcal{M}_{\mathbb{k}}}=U\left(\mathfrak{g}_{\mathbb{k}}, e\right)$ and $Z_{p}\left(\widetilde{\mathfrak{a}}_{\mathbb{k}}\right) \cap U\left(\mathfrak{g}_{\mathbb{k}}, e\right)=Z_{p}\left(\widetilde{\mathfrak{a}}_{\mathbb{k}}\right)^{\mathcal{M}_{\mathrm{k}}}$. On the other hand, [31, Lemma 3.2] entails that each fibre of the categorical quotient $\chi+\mathfrak{m}_{\mathbb{k}}^{\perp} \rightarrow\left(\chi+\mathfrak{m}_{\mathbb{k}}^{\perp}\right) / / \mathcal{M}_{\mathbb{k}}$ induced by inclusion $\mathbb{k}\left[\chi+\mathfrak{m}_{\mathbb{k}}^{\perp}\right]^{\mathcal{M}_{\mathrm{k}}} \hookrightarrow \mathbb{k}\left[\chi+\mathfrak{m}_{\mathbb{k}}^{\perp}\right]$ is a single $\mathcal{M}_{\mathbb{k}}$-orbit. As the maximal spectrum of $Z_{p}\left(\widetilde{\mathfrak{a}}_{\mathbb{k}}\right)$ is isomorphic to $\left(\chi+\mathfrak{m}_{\mathbb{k}}^{\perp}\right)^{(1)}$ as $\mathcal{M}_{\mathbb{k}^{\mathrm{k}}}$-varieties by our earlier remarks, each fibre of the categorical quotient

$$
\alpha: \operatorname{Specm} Z_{p}\left(\widetilde{\mathfrak{a}}_{\mathbb{k}}\right) \longrightarrow\left(\operatorname{Specm} Z_{p}\left(\widetilde{\mathfrak{a}}_{\mathbb{k}}\right)\right) / / \mathcal{M}_{\mathbb{k}}
$$

is a single $\mathcal{M}_{\mathbb{k}^{\mathrm{k}}}$-orbit as well. Now let $\Psi_{i}, i=1,2$, be two linear functions as above, denote by $\psi_{i}$ the restriction of $\Psi_{i}$ to $\mathfrak{a}_{\mathbb{k}}$, and consider the corresponding representations $\widehat{\tau}_{\mathbb{k}, \psi_{i}}: \widehat{U}\left(\mathfrak{g}_{\mathbb{k}}, e\right) \rightarrow$ End $M_{\mathbb{k}}$. Since $\widehat{\tau}_{\mathbb{k}, \psi_{1}}$ and $\widehat{\tau}_{\mathbb{k}, \psi_{2}}$ agree on $Z_{p}\left(\widetilde{\mathfrak{a}}_{\mathbb{k}}\right)^{\mathcal{M} \mathcal{M}_{\mathrm{k}}} \subset U\left(\mathfrak{g}_{\mathbb{k}}, e\right)$, it must be that $\alpha\left(\Psi_{1}\right)=\alpha\left(\Psi_{2}\right)$. But then $\Psi_{1}$ and $\Psi_{2}$ are in the same $\mathcal{M}_{\mathbb{k}}$-orbit, as claimed.

The above discussion in conjunction with [31, Lemma 3.2] also yields that for $p \gg 0$ the central subalgebra $Z_{p}(\widetilde{\mathfrak{a}})^{\mathcal{M}_{\mathbb{k}}} \cong \mathbb{k}\left[\left(\chi+\mathfrak{m}_{\mathbb{k}}^{\perp}\right)^{(1)}\right]^{\mathcal{M}_{\mathfrak{k}}}$ of $U\left(\mathfrak{g}_{\mathbb{k}}, e\right)$ is isomorphic to the function algebra on the Frobenius twist of the Slodowy slice $\mathbb{S}_{\chi}:=\chi+\kappa(\operatorname{Ker} \operatorname{ad} f)$, where $\kappa: \mathfrak{g}_{\mathbb{k}} \stackrel{\sim}{\longrightarrow} \mathfrak{g}_{\mathbb{k}}^{*}$ is the Killing isomorphism associated with the bilinear form $(\cdot, \cdot)$. Together with [31, Thm. 2.1] this shows that the relationship between $U_{\eta}\left(\mathfrak{g}_{\mathbb{k}}, e\right)$ and $U\left(\mathfrak{g}_{\mathrm{k}}, e\right)$ is very similar to that between $U_{\eta}\left(\mathfrak{g}_{\mathrm{k}}\right)$ and $U\left(\mathfrak{g}_{\mathrm{k}}\right)$. More precisely, one embeds $\mathbb{k}\left[\left(\mathbb{S}_{\chi}\right)^{(1)}\right]$ into $U\left(\mathfrak{g}_{\mathbb{k}}, e\right)$ as an analogue of the $p$-centre $Z_{p}\left(\mathfrak{g}_{\mathbb{k}}\right)$ (so that $U\left(\mathfrak{g}_{\mathbb{k}}, e\right)$ is a free $\mathbb{k}\left[\left(\mathbb{S}_{\chi}\right)^{(1)}\right]$-module of rank $\left.p^{r}\right)$ and then obtains $U_{\eta}\left(\mathfrak{g}_{\mathbb{k}}, e\right)$ from $U\left(\mathfrak{g}_{\mathbb{k}}, e\right)$ by tensoring the latter over $\mathbb{k}\left[\left(\mathbb{S}_{\chi}\right)^{(1)}\right]$ by a suitable one-dimensional representation of $\mathbb{k}\left[\left(\mathbb{S}_{\chi}\right)^{(1)}\right]$. 
2.7. Put $\mathcal{J}_{A}:=\operatorname{Ann}_{U\left(\mathfrak{g}_{A}\right)} \widetilde{M}_{A}$ and denote by $\operatorname{gr}\left(\mathcal{J}_{A}\right)$ the corresponding graded ideal of $S\left(\mathfrak{g}_{A}\right)$. Define $R:=U(\mathfrak{g}) / \mathcal{J}, \operatorname{gr}(R):=S(\mathfrak{g}) / \operatorname{gr}(\mathcal{J}), R_{A}:=U\left(\mathfrak{g}_{A}\right) / \mathcal{J}_{A}$, and $\operatorname{gr}\left(R_{A}\right)=$ $S\left(\mathfrak{g}_{A}\right) / \operatorname{gr}\left(\mathcal{J}_{A}\right)$. Clearly, $\operatorname{gr}\left(R_{A}\right)=\bigoplus_{n \geq 0}\left(\operatorname{gr}\left(R_{A}\right)\right)(n)$ is a finitely generated graded $A$-algebra and each $\left(\operatorname{gr}\left(R_{A}\right)\right)(n)$ is a finitely generated $A$-module. Also, $A$ is a commutative Noetherian domain. If $b \in A \backslash\{0\}$, then $\operatorname{gr}\left(\mathcal{J}_{A\left[b^{-1}\right]}\right)=\operatorname{gr}\left(\mathcal{J}_{A}\right) \otimes_{A} A\left[b^{-1}\right]$ and

$$
\begin{aligned}
\operatorname{gr}\left(R_{A\left[b^{-1}\right]}\right) & =S\left(\mathfrak{g}_{A\left[b^{-1}\right]}\right) / \operatorname{gr}\left(\mathcal{J}_{A\left[b^{-1}\right]}\right) \cong\left(S\left(\mathfrak{g}_{A}\right) \otimes_{A} A\left[b^{-1}\right]\right) /\left(\operatorname{gr}\left(\mathcal{J}_{A}\right) \otimes_{A} A\left[b^{-1}\right]\right) \\
& \cong \operatorname{gr}\left(R_{A}\right) \otimes_{A} A\left[b^{-1}\right] ;
\end{aligned}
$$

see [4, Ch. II, 2.4], for example. Since $\operatorname{gr}(R)=\bigoplus_{n \geq 0}(\operatorname{gr}(R))(n)$ is a graded Noetherian algebra of Krull dimension $2 d(e)=\operatorname{dim} \mathcal{O}$ with $(\operatorname{gr}(R))(0)=\mathbb{C}$, we have that $2 d(e)=\operatorname{dim} \operatorname{gr}(R)=1+\operatorname{deg} P_{R}(t)$, where $P_{\operatorname{gr}(R)}(t)$ is the Hilbert polynomial of $\operatorname{gr}(R)$; see [10, Corollary 13.7].

Denote by $F$ the quotient field of $A$. Since $\operatorname{gr}\left(R_{F}\right):=\operatorname{gr}\left(R_{A}\right) \otimes_{A} F$ is a finitely generated algebra over a field, the Noether Normalisation Theorem says that there exist homogeneous, algebraically independent $y_{1}, \ldots, y_{2 d(e)} \in \operatorname{gr}(R)_{F}$, such that $\operatorname{gr}\left(R_{F}\right)$ is a finitely generated module over its graded polynomial subalgebra $F\left[y_{1}, \ldots, y_{2 d(e)}\right]$; see $\left[10\right.$, Thm. 13.3]. Let $v_{1}, \ldots, v_{D}$ be a generating set of the $F\left[y_{1}, \ldots, y_{2 d(e)}\right]$-module $\operatorname{gr}\left(R_{F}\right)$ and let $r_{1}, \ldots, r_{N}$ be a generating set of the $A$-algebra $\operatorname{gr}\left(R_{A}\right)$. Then

$$
\begin{aligned}
v_{i} \cdot v_{j} & =\sum_{k=1}^{D} p_{i, j}^{k}\left(y_{1}, \ldots, y_{d(e)}\right) v_{k} & & (1 \leq i, j \leq D) \\
r_{i} & =\sum_{j=1}^{D} q_{i, j}\left(y_{1}, \ldots, y_{d(e)}\right) v_{j} & & (1 \leq i \leq N)
\end{aligned}
$$

for some polynomials $p_{i, j}^{k}, q_{i, j} \in F\left[X_{1}, \ldots, X_{2 d(e)}\right]$. The algebra $\operatorname{gr}\left(R_{A}\right)$ contains an $F$-basis of $\operatorname{gr}\left(R_{F}\right)$. The coordinate vectors of the $r_{i}$ 's, $y_{i}$ 's and $v_{i}$ 's relative to this basis and the coefficients of the polynomials $q_{i, j}$ and $p_{i, j}^{k}$ involve only finitely many scalars in $F$. Replacing $A$ by $A\left[b^{-1}\right]$ for a suitable $0 \neq b \in A$ if necessary, we may assume that all $y_{i}$ and $v_{i}$ are in $\operatorname{gr}\left(R_{A}\right)$ and all $p_{i, j}^{k}$ and $q_{i, j}$ are in $A\left[X_{1}, \ldots, X_{2 d(e)}\right]$. In conjunction with our earlier remarks this shows that no generality will be lost by assuming that

$$
\operatorname{gr}\left(R_{A}\right)=A\left[y_{1}, \ldots, y_{2 d(e)}\right] v_{1}+\cdots+A\left[y_{1}, \ldots, y_{2 d(e)}\right] v_{D}
$$

is a finitely generated module over the polynomial algebra $A\left[y_{1}, \ldots, y_{2 d(e)}\right]$.

Since $\operatorname{gr}\left(R_{A}\right)$ is a finitely generated $A\left[y_{1}, \ldots, y_{d(e)}\right]$-module and $A$ is a Noetherian domain, a graded version of the Generic Freeness Lemma shows that there exists a nonzero element $a_{1} \in A$ such that each $\left(\operatorname{gr}\left(R_{A}\right)(n)\right)\left[a_{1}^{-1}\right]$ is a free $A\left[a_{1}^{-1}\right]$-module of finite rank; see (the proof of) Theorem 14.4 in [10]. Since $\left(\operatorname{gr}\left(R_{A}\right)(n)\right)\left[a_{1}^{-1}\right] \cong$ $\left(\operatorname{gr}\left(R_{A\left[a_{1}^{-1}\right]}\right)\right)(n)$ for all $n$ by our earlier remarks, we see that there exists an admissible ring $A \subset \mathbb{C}$ such that all graded components of $\operatorname{gr}\left(R_{A}\right)$ are free $A$-modules of finite rank.

Since $S\left(\mathfrak{g}_{A}\right)$ is a finitely generated $A$-algebra, we can also apply the proof of Theorem 14.4 in [10] to the graded ideal $\operatorname{gr}\left(\mathcal{J}_{A}\right)$ of $S\left(\mathfrak{g}_{A}\right)$ to deduce that there exists a nonzero $a_{2} \in A$ such that all graded components of $\left(\operatorname{gr}\left(\mathcal{J}_{A}\right)\right)\left[a_{2}^{-1}\right]$ are free $A\left[a_{2}^{-1}\right]$ modules of finite rank. As $\left(\operatorname{gr}\left(\mathcal{J}_{A}\right)\right)\left[a_{2}^{-1}\right] \cong \operatorname{gr}\left(\mathcal{J}_{A\left[a_{2}^{-1}\right]}\right)$ by $[4$, Ch. II, 2.4], we may (and we will) assume that all graded components of $\operatorname{gr}\left(\mathcal{J}_{A}\right)$ are free $A$-modules of 
finite rank. Using the standard filtered-graded techniques we now obtain that the $A$-modules $\mathcal{J}_{A}$ and $R_{A}$ are free as well.

2.8. Note that $\widetilde{M}_{F}=\widetilde{M}_{A} \otimes_{A} F$ is a module over the split Lie algebra $\mathfrak{g}_{F}$. Since $\widetilde{M} \cong \widetilde{M}_{F} \otimes_{F} \mathbb{C}$, each subspace $\mathcal{J} \cap U_{k}(\mathfrak{g})$ is defined over $F$ (here $U_{k}(\mathfrak{g})$ stands for the $k$ th component of the canonical filtration of $U(\mathfrak{g}))$. Since the algebra $U(\mathfrak{g})$ is Noetherian, the ideal $\mathcal{J}$ is generated by its $F$-subspace $\mathcal{J}_{F, N}:=U_{N}\left(\mathfrak{g}_{F}\right) \cap \mathcal{J}$. Since $\mathcal{J}$ is a two-sided ideal of $U(\mathfrak{g})$, all subspaces $\mathcal{J} \cap U_{k}(\mathfrak{g})$ are invariant under the adjoint action of $G$ on $U(\mathfrak{g})$. Hence the $F$-subspace $\mathcal{J}_{F, N}$ is invariant under the adjoint action of the distribution algebra $U_{F}:=U_{\mathbb{Z}} \otimes_{\mathbb{Z}} F$. Since $\mathfrak{h}_{F}:=\mathfrak{h} \cap \mathfrak{g}_{F}$ is a split Cartan subalgebra of $\mathfrak{g}_{F}$, the adjoint $\mathfrak{g}_{F}$-module $\mathfrak{J}_{F, N}$ decomposes into a finite direct sum of absolutely irreducible $\mathfrak{g}_{F}$-modules with integral dominant highest weights. Therefore, the $\mathfrak{g}_{F^{-}}$ module $\mathcal{J}_{F, N}$ possesses a $\mathbb{Z}$-form invariant under the adjoint action of the Kostant $\mathbb{Z}$-form $U_{\mathbb{Z}}$; we call it $\mathcal{J}_{\mathbb{Z}, N}$.

Let $\left\{u_{i} \mid i \in I\right\}$ be any basis of the free $\mathbb{Z}$-module $\mathcal{J}_{\mathbb{Z}, N}$. Expressing the $u_{i}$ via the PBW basis of $U\left(\mathfrak{g}_{F}\right)$ associated with the Chevalley basis $\mathcal{B}$ involves only finitely many scalars in $F$. Enlarging $A$ further if need be we may assume that all $u_{i}$ are in $U\left(\mathfrak{g}_{A}\right)$ and hence that the ideal $\mathcal{J}_{A}$ of $U\left(\mathfrak{g}_{A}\right)$ is invariant under the natural action of the Hopf $\mathbb{Z}$-algebra $U_{\mathbb{Z}}$ (one should keep in mind here that the $\mathbb{Z}$-algebra $U_{\mathbb{Z}}$ is generated by all $e_{\gamma}^{n} / n$ ! with $\gamma \in \Phi$ and $\left.n \in \mathbb{Z}_{+}\right)$. Thus, from now on we may assume that for any maximal ideal $\mathfrak{P}$ of $A$ the two-sided ideal $\mathcal{J}_{\mathbb{k}}:=\mathcal{J}_{A} \otimes_{A} \mathbb{k}_{\mathfrak{P}}$ of $U\left(\mathfrak{g}_{\mathbb{k}}\right)$ is stable under the adjoint action of the simple algebraic $\mathbb{k}$-group $G_{\mathbb{k}}$ with hyperalgebra $U_{\mathbb{k}}=U_{\mathbb{Z}} \otimes_{\mathbb{Z}} \mathbb{k}$.

\section{Introducing certain finite subsets of regular elements in $R$}

3.1. Let $\mathcal{B}=\left\{g_{1}, \ldots, g_{n}\right\}$ be our Chevalley basis of $\mathfrak{g}_{\mathbb{Z}}$ and identify $\mathcal{B}$ with its image in $R=U(\mathfrak{g}) / \mathcal{J}$. Denote by $R_{k}$ the $k$ th component of the filtration of $R$ induced by the canonical filtration of $U(\mathfrak{g})$ and let $S$ be the Ore set of all regular elements in $R$. Since $\mathcal{Q}(R)=S^{-1} R \cong \operatorname{Mat}_{l^{\prime}}\left(\mathcal{D}_{M}\right)$, where $l^{\prime}=\operatorname{rk}(R)$, there exists a unital subalgebra $\mathfrak{C}$ in $\mathcal{Q}(R)$ isomorphic to $\operatorname{Mat}_{l^{\prime}}(\mathbb{C})$ and such that $\mathcal{Q}(R) \cong \mathfrak{C} \otimes \mathfrak{D}$, where $\mathfrak{D} \cong \mathcal{D}_{M}$ is the centraliser of $\mathfrak{C}$ in $\mathcal{Q}(R)$.

In this section, we are going to describe an algorithmic procedure that will produce at the end certain finite subsets $X \subset R$ and $Y \subset S$. The subalgebra of $\mathcal{Q}(R)$ generated by $X$ and by $Y \cup Y^{-1}$ will contain the Chevalley basis $\mathcal{B}$, a fixed set of matrix units of $\mathfrak{C}$ and a generating set of the skew-field $\mathfrak{C}$. The actual form of the elements in $X$ and $Y$ will be of no importance for us, but in the next section we will rely on the fact that our procedure is finite and each of its steps is reversible.

Fix a set $\left\{e_{i j} \mid 1 \leq i, j \leq l^{\prime}\right\}$ of matrix units in $\mathfrak{C}$, so that

$$
\begin{aligned}
e_{i j} e_{t k} & =\delta_{j t} e_{i k} \\
\sum_{i=1}^{l^{\prime}} e_{i i} & =1 .
\end{aligned} \quad\left(1 \leq i, j, t, k \leq l^{\prime}\right) ;
$$

There exist $s_{i j}, s_{i j}^{\prime} \in S$ and $E_{i j}, E_{i j}^{\prime} \in R$ such that

$$
s_{i j}^{-1} E_{i j}=e_{i j}=E_{i j}^{\prime}\left(s_{i j}^{\prime}\right)^{-1} \text {. }
$$

Then in $R$ we have the following relations

$$
E_{i j} s_{i j}^{\prime}=s_{i j} E_{i j}^{\prime} \quad\left(1 \leq i, j \leq l^{\prime}\right) .
$$


As $\mathcal{Q}(R)=\mathfrak{C} \otimes \mathfrak{D}$, there exist $c_{i j}^{k} \in \mathcal{Q}(R)$, where $1 \leq k \leq n$, such that

$$
\begin{aligned}
g_{k} & =\sum_{i, j} e_{i j} c_{i j}^{k} & & (1 \leq k \leq n) ; \\
c_{i j}^{k} e_{t h} & =e_{t h} c_{i j}^{k} & & \left(1 \leq i, j, t, h \leq l^{\prime} ; 1 \leq k \leq n\right) .
\end{aligned}
$$

For each $k \leq l^{\prime}$ we can find $a_{i j}^{k} \in S$ and $C_{i j}^{k} \in R$ such that $c_{i j}^{k}=\left(a_{i j}^{k}\right)^{-1} C_{i j}^{k}$. Since $S$ is an Ore set, there are $r_{i j, t k}, r_{i j, t h}^{k}, a_{i j, t h}^{k} \in S$ and $E_{i j, t k}, E_{i j, t h}^{k}, C_{i j, t h}^{k} \in R$ such that

$$
\begin{aligned}
r_{i j, t k} E_{i j} & =E_{i j, t k} s_{t k} & & \left(1 \leq i, j, t, k \leq l^{\prime}\right) ; \\
r_{i j, t h}^{k} C_{i j}^{k} & =E_{i j, t h}^{k} s_{t h} & & \left(1 \leq i, j, t, h \leq l^{\prime}, 1 \leq k \leq l^{\prime}\right) ; \\
C_{i j}^{k} a_{i j, t h}^{k} & =a_{i j}^{k} s_{t h}^{\prime} C_{i j, t h}^{k} & & \left(1 \leq i, j, t, h \leq l^{\prime}, 1 \leq k \leq l^{\prime}\right) .
\end{aligned}
$$

Since $s_{i j}^{-1} E_{i j} s_{t k}^{-1} E_{t k}=\delta_{j t} E_{i k}^{\prime}\left(s_{i k}^{\prime}\right)^{-1}$ by (6) and (9), applying (12) we obtain that $s_{i j}^{-1} r_{i j, t k}^{-1} E_{i j, t k} E_{t k}=\delta_{j t} E_{i k}^{\prime}\left(s_{i k}^{\prime}\right)^{-1}$. This yields

$$
E_{i j, t k} E_{i k} s_{i k}^{\prime}=\delta_{j t} r_{i j, t k} s_{i j} E_{i k}^{\prime} \quad\left(1 \leq i, j, t, k \leq l^{\prime}\right) .
$$

Similarly, since $\left(a_{i j}^{k}\right)^{-1} C_{i j}^{k} s_{t h}^{-1} E_{t h}=E_{t h}^{\prime}\left(s_{t h}^{\prime}\right)^{-1}\left(a_{i j}^{k}\right)^{-1} C_{i j}^{k}$ by (11) and (9), applying (13) and (14) yields $\left(a_{i j}^{k}\right)^{-1}\left(r_{i j, t h}^{k}\right)^{-1} E_{i j, t h}^{k} E_{t h}=E_{t h}^{\prime} C_{i j, t h}^{k}\left(a_{i j, t h}^{k}\right)^{-1}$. We thus get

$$
E_{i j, t h}^{k} E_{t h} a_{i j, t h}^{k}=r_{i j, t h}^{k} a_{i j}^{k} E_{t h}^{\prime} C_{i j, t h}^{k} \quad\left(1 \leq i, j, t, h \leq l^{\prime}, 1 \leq k \leq l^{\prime}\right) .
$$

Recall that $1=\sum_{i=1}^{l^{\prime}} e_{i i}=\sum_{i=1}^{l^{\prime}} s_{i i}^{-1} E_{i i}$. Multiplying both sides by $s_{11}$ on the left we get

$$
s_{11}=E_{11}+\sum_{i=2}^{l^{\prime}} s_{11} s_{i i}^{-1} E_{i i} .
$$

There exist $s_{1,2} \in S$ and $q_{2} \in R$ such that $s_{1,2} s_{11}=q_{2} s_{22}$. Multiplying both sides of (17) by $s_{1,2}$ on the left we then obtain

$$
s_{1,2} s_{11}=s_{1,2} E_{11}+q_{2} E_{22}+\sum_{i=3}^{l^{\prime}} s_{1,2} s_{11} s_{i i}^{-1} E_{i i} .
$$

For $3 \leq k \leq l^{\prime}$, we select (recursively) some $s_{1, \ldots, k} \in S$ and $q_{k} \in R$ such that

$$
\prod_{i=1}^{k} s_{1, \ldots, k-i+1}=q_{k} s_{k k} .
$$

For convenience, we set $q_{1}=1$. At the end of the process started with (17) and (18) we get rid of all denominators and arrive at the relation

$$
\prod_{k=1}^{l^{\prime}} s_{1, \ldots, l^{\prime}-k+1}=\sum_{k=1}^{l^{\prime}}\left(\prod_{i=1}^{l^{\prime}-k} s_{1, \ldots, l^{\prime}-i+1}\right) q_{k} E_{k k}
$$

which holds in $R$.

Let $p(1), \ldots, p\left(l^{2}\right)$ be all elements in the lexicographically ordered set $\{(i, j) \mid 1 \leq$ $\left.i, j \leq l^{\prime 2}\right\}$ and denote by $e_{p(k)}, E_{p(k)}$ and $s_{p(k)}$ the corresponding elements in $R$. Since $e_{i j}$ commutes with $c_{i j}^{k}$ we can rewrite (10) as

$$
g_{k}=\sum_{i, j}\left(a_{i j}^{k}\right)^{-1} C_{i j}^{k} s_{i j}^{-1} E_{i j} \quad(1 \leq k \leq n) .
$$

For $1 \leq k \leq l^{\prime}$, there exist $D_{i j}^{k} \in R$ and $s_{i j}^{k} \in S$ such that

$$
D_{i j}^{k} s_{i j}=s_{i j}^{k} C_{i j}^{k} \quad\left(1 \leq i, j \leq l^{\prime}\right) .
$$


Then, setting $T_{i j}^{k}:=D_{i j}^{k} E_{i j}$ and $s_{i j ; k}:=s_{i j}^{k} a_{i j}^{k}$, we can rewrite (21) as follows:

$$
g_{k}=\sum_{i, j} s_{i j ; k}^{-1} T_{i j}^{k}=\sum_{i=1}^{l^{\prime 2}} s_{p(i) ; k}^{-1} T_{p(i)}^{k} \quad(1 \leq k \leq n) .
$$

Multiplying both sides of $(23)$ by $s_{p(1) ; k}$ on the left we get

$$
s_{p(1) ; k} \cdot g_{k}=T_{p(1)}^{k}+\sum_{i=2}^{l^{\prime 2}} s_{p(1) ; k} s_{p(i) ; k}^{-1} T_{p(i)} .
$$

There are $s_{p(1), p(2) ; k} \in S$ and $q_{p(2)}^{k} \in R$ such that $s_{p(1), p(2) ; k} s_{p(1) ; k}=q_{p(2)}^{k} s_{p(2) ; k}$. Multiplying both sides of (24) by $s_{p(1), p(2) ; k}$ on the left we get

$$
\left(s_{p(1), p(2) ; k} s_{p(1) ; k}\right) g_{k}=s_{p(1), p(2) ; k} T_{p(1)}^{k}+q_{p(2)}^{k} T_{p(2)}^{k}+\sum_{i=3}^{l^{\prime 2}} s_{p(1), p(2) ; k} s_{p(1) ; k} s_{p(i) ; k}^{-1} T_{p(i)}^{k} .
$$

For $3 \leq j \leq l^{\prime 2}$, we choose (recursively) some $s_{p(1), \ldots, p(j) ; k} \in S$ and $q_{p(j)}^{k} \in R$ such that

$$
\prod_{i=1}^{j} s_{p(1), \ldots, p(j-i+1) ; k}=q_{p(j)}^{k} s_{p(j) ; k}
$$

and set $q_{p(1)}^{k}=1$. As before, at the end of the process just started we arrive at the relations

$$
\left(\prod_{j=1}^{l^{\prime 2}} s_{p(1), \ldots, p\left(l^{\prime 2}-j+1\right) ; k}\right) g_{k}=\sum_{j=1}^{l^{\prime 2}}\left(\prod_{i=1}^{l^{\prime 2}-j} s_{p(1), \ldots, p\left(l^{\prime 2}-i+1\right) ; k}\right) q_{p(j)}^{k} T_{p(j)}^{k}
$$

which hold in $R$, where $1 \leq k \leq n$.

We now denote by $X$ the set of all elements

$$
E_{i j}, E_{i j}^{\prime}, C_{i j}^{k}, E_{i j, t k}, E_{i j, t h}^{k}, C_{i j, t h}^{k}, q_{k}, D_{i j}^{k}, T_{i j}^{k}, q_{p(j) ; k}
$$

and by $Y$ the set of all elements

$$
s_{i j}, s_{i j}^{\prime}, a_{i j}^{k}, r_{i j, t k}, r_{i j, t h}^{k}, a_{i j, t h}^{k}, s_{1, \ldots, k}, s_{i j}^{k}, s_{i j ; k}, s_{p(1), \ldots, p(j) ; k} .
$$

3.2. In this subsection we assume that $\mathfrak{D}$ is a Weyl skew-field, more precisely, $\mathfrak{D} \cong$ $\mathcal{Q}\left(\mathbf{A}_{d(e)}(\mathbb{C})\right)$. We follow closely the exposition in [30, Sect. 2] and adopt (with some minor modifications) the notation introduced there.

Set $d:=d(e)$. If a pair $(a, b) \in\left\{(i, j) \mid 1 \leq i, j \leq l^{\prime}\right\}$ occupies the $k$ th place in our lexicographical ordering, then we write $c_{p(k)}^{s}, a_{p(k)}^{s}$ and $C_{p(k)}^{s}$ for $c_{a b}^{s}, a_{a b}^{s}$ and $C_{a b}^{s}$, respectively. There exist $w_{1}, \ldots, w_{2 d} \in \mathfrak{D}$ such that

$$
\begin{aligned}
{\left[w_{i}, w_{j}\right]=\left[w_{d+i}, w_{d+j}\right] } & =0 & & (1 \leq i, j \leq d) ; \\
{\left[w_{i}, w_{d+j}\right] } & =\delta_{i, j} & & (1 \leq i, j \leq d) ; \\
Q_{p(k)}^{s} \cdot c_{p(k)}^{s} & =P_{p(k)}^{s}, & & \left(1 \leq k \leq l^{\prime 2} ; 1 \leq s \leq n\right)
\end{aligned}
$$

for some nonzero polynomials $P_{p(k)}^{s}, Q_{p(k)}^{s}$ in $w_{1}, \ldots, w_{2 d}$ with coefficients in $\mathbb{C}$. (One should keep in mind here that the monomials $w_{1}^{a_{1}} w_{2}^{a_{2}} \cdots w_{2 d}^{a_{2 d}}$ with $a_{i} \in \mathbb{Z}_{+}$form a basis of the $\mathbb{C}$-subalgebra of $D$ generated by $w_{1}, \ldots, w_{2 d}$.)

Since every nonzero element of $\mathfrak{D}$ is regular in $\mathcal{Q}(R)$, there exist $Q_{1 ; p(k)}^{s}, Q_{2 ; p(k)}^{s} \in S$ such that

$$
Q_{p(k)}^{s} Q_{1 ; p(k)}^{s}=Q_{2 ; p(k)}^{s} \quad\left(1 \leq k \leq l^{\prime 2} ; 1 \leq s \leq n\right) .
$$


Since $w_{i}=v_{i}^{-1} u_{i}$ for some elements $v_{i} \in S$ and $u_{i} \in R$, we can rewrite (27) and (28) as follows:

$$
\begin{aligned}
v_{i}^{-1} u_{i} \cdot v_{j}^{-1} u_{j} & =v_{j}^{-1} u_{j} \cdot v_{i}^{-1} u_{i} ; \\
v_{d+i}^{-1} u_{d+i} \cdot v_{d+j}^{-1} u_{d+j} & =v_{d+j}^{-1} u_{d+j} \cdot v_{d+i}^{-1} u_{d+i} ; \\
v_{i}^{-1} u_{i} \cdot v_{d+j}^{-1} u_{d+j}-v_{d+j}^{-1} u_{d+j} \cdot v_{i}^{-1} u_{i} & =\delta_{i, j} \quad(1 \leq i, j \leq d) .
\end{aligned}
$$

As $S$ is an Ore set, there are $v_{i, j} \in S$ and $u_{i, j} \in R$ such that

$$
v_{i, j} u_{i}=u_{i, j} v_{j} \quad(1 \leq i, j \leq 2 d) .
$$

Thus we can rewrite (31), (32) and (33) in the form

$$
\begin{aligned}
v_{i}^{-1} v_{i, j}^{-1} \cdot u_{i, j} u_{j} & =v_{j}^{-1} v_{j, i}^{-1} \cdot u_{j, i} u_{i} \quad(1 \leq i, j \leq d \text { or } d \leq i, j \leq 2 d) \\
v_{i}^{-1} v_{i, d+j}^{-1} \cdot u_{i, d+j} u_{d+j} & =\delta_{i j}+v_{d+j}^{-1} v_{d+j, i}^{-1} \cdot u_{d+j, i} u_{d+i} \quad(1 \leq i, j \leq d) .
\end{aligned}
$$

There exist $b_{i, j} \in S$ and $b_{i, j}^{\prime} \in R$ such that

$$
b_{i, j} v_{i, j} v_{i}=b_{i, j}^{\prime} v_{j, i} v_{j} \quad(1 \leq i, j \leq 2 d) .
$$

Since $v_{i, j} v_{i}\left(v_{j, i} v_{j}\right)^{-1}=b_{i, j}^{-1} b_{i, j}^{\prime}$, we see that (35) and (36) give rise to the relations

$$
\begin{aligned}
b_{i, j} u_{i, j} u_{j} & =b_{i, j}^{\prime} u_{j, i} u_{i} \quad(1 \leq i, j \leq d \text { or } d \leq i, j \leq 2 d) \\
b_{i, d+j} u_{i, d+j} u_{d+j} & =\delta_{i j} b_{i, d+j} v_{i, d+j} v_{i}+b_{i, d+j}^{\prime} u_{d+j, i} u_{i} \quad(1 \leq i, j \leq d)
\end{aligned}
$$

which hold in $R$.

For an $m$-tuple $\mathbf{i}=(i(1), i(2), \ldots, i(m))$ with $1 \leq i(1) \leq i(2) \leq \cdots \leq i(m) \leq 2 d$ and $m \geq 3$ we select (recursively) some $u_{i(1), \ldots, i(k)} \in R$ and $v_{i(1), \ldots, i(k)} \in S$, where $3 \leq k \leq m$, such that

$$
v_{i(1), \ldots, i(k)} u_{i(1), \ldots, i(k-1)} u_{i(k-1)}=u_{i(1), \ldots, i(k)} v_{i(k)} .
$$

Write $w^{\mathbf{i}}:=w_{i(1)} \cdot w_{i(2)} \cdot \ldots \cdot w_{i(m)}=\prod_{k=1}^{m} v_{i(k)}^{-1} u_{i(k)}$. Then

$$
\begin{aligned}
w^{\mathrm{i}} & =v_{i(1)}^{-1} u_{i(1)} \cdot v_{i(2)}^{-1} u_{i(2)} \cdot \prod_{k=3}^{m} v_{i(k)}^{-1} u_{i(k)} \\
& =v_{i(1)}^{-1} v_{i(1), i(2)}^{-1} u_{i(1), i(2)} u_{i(2)} \cdot v_{i(3)}^{-1} u_{i(3)} \cdot \prod_{k=4}^{m} v_{i(k)}^{-1} u_{i(k)} \\
& =v_{i(1)}^{-1} v_{i(1), i(2)}^{-1} v_{i(1), i(2), i(3)}^{-1} u_{i(1), i(2), i(3)} u_{i(3)} \cdot \prod_{k=4}^{m} v_{i(k)}^{-1} u_{i(k)} \\
& =\cdots=\left(\prod_{k=1}^{m} v_{i(1), \ldots, i(m-k+1)}\right)^{-1} \cdot u_{i(1), \ldots, i(m)} u_{i(m)} .
\end{aligned}
$$

Then we set $v_{\mathbf{i}}:=\prod_{k=1}^{m} v_{i(1), \ldots, i(m-k+1)}$, an element of $S$, and $u_{\mathbf{i}}:=u_{i(1), \ldots, i(m)} u_{i(m)}$, an element of $R$.

Let $\{\mathbf{i}(1), \ldots, \mathbf{i}(N)\}$ be the set of all tuples as above with $\sum_{\ell=1}^{N} i(\ell) \leq \Delta$, where $\Delta=$ $\max \left\{\operatorname{deg} P_{p(k)}^{s}, \operatorname{deg} Q_{p(k)}^{s} \mid 1 \leq k \leq l^{\prime 2}, 1 \leq s \leq n\right\}$. Clearly, $P_{p(k)}^{s}=\sum_{j=1}^{N} \lambda_{j, k}^{s} w^{\mathbf{i}(j)}$ and $Q_{p(k)}^{s}=\sum_{j=1}^{N} \mu_{j, k}^{s} w^{\mathrm{i}(j)}$ for some $\lambda_{j, k}^{s}, \mu_{j, k}^{s} \in \mathbb{C}$, where $1 \leq k \leq l^{\prime 2}$ and $1 \leq s \leq n$. By the above, we have that $P_{p(k)}^{s}=\sum_{j=1}^{N} \lambda_{j, k}^{s} v_{\mathbf{i}(j)}^{-1} u_{\mathbf{i}(j)}$ and $Q_{p(k)}^{s}=\sum_{i=1}^{N} \mu_{j, k}^{s} v_{\mathbf{i}(j)}^{-1} u_{\mathbf{i}(j)}$. 
Set $v_{\mathbf{i}(j)}(0):=v_{\mathbf{i}(j)}$ and $u_{\mathbf{i}(j)}(0)=u_{\mathbf{i}(j)}$. For each pair $(j, t)$ of positive integers satisfying $N \geq j>t>0$ we select (recursively) some $v_{\mathbf{i}(j)}(t) \in S$ and $u_{\mathbf{i}(j)}(t) \in R$ such that

$$
v_{\mathbf{i}(j)}(t) v_{\mathbf{i}(t)}(t-1)=u_{\mathbf{i}(j)}(t) v_{\mathbf{i}(j)}(t-1) .
$$

Multiplying both sides of (29) by $v_{\mathbf{i}(1)}$ on the left and applying (41) with $t=1$ we obtain that

$$
\begin{aligned}
v_{\mathbf{i}(1)} P_{p(k)}^{s} & =\lambda_{1, k}^{s} u_{\mathbf{i}(1)}+\sum_{j=2}^{N} \lambda_{j, k}^{s} v_{\mathbf{i}(1)} v_{\mathbf{i}(j)}^{-1} u_{\mathbf{i}(j)} \\
& =\lambda_{1, k}^{s} u_{\mathbf{i}(1)}+\sum_{j=2}^{N} \lambda_{j, k}^{s} v_{\mathbf{i}(j)}(1)^{-1} u_{\mathbf{i}(j)}(1) u_{\mathbf{i}(j)} .
\end{aligned}
$$

Multiplying both sides of this equality by $v_{\mathbf{i}(2)}(1)$ on the left and applying (41) with $t=2$ we get

$$
\begin{aligned}
v_{\mathbf{i}(2)}(1) v_{\mathbf{i}(1)} P_{p(k)}^{s} & =\lambda_{1, k}^{s} v_{\mathbf{i}(2)}(1) u_{\mathbf{i}(1)}+\lambda_{2, k}^{s} u_{\mathbf{i}(2)}(1) u_{\mathbf{i}(1)} \\
& +\sum_{j=3}^{N} \lambda_{j, k}^{s} v_{\mathbf{i}(j)}(2)^{-1} u_{\mathbf{i}(j)}(2) u_{\mathbf{i}(j)}(1) u_{\mathbf{i}(j)} .
\end{aligned}
$$

Repeating this process $N$ times we arrive at the relation

$$
\left(\prod_{\ell=1}^{N} v_{\mathbf{i}(N-\ell+1)}\right) P_{p(k)}^{s}=\sum_{j=1}^{N} \lambda_{j, k}^{s} \cdot\left(\prod_{\ell=1}^{N-j} v_{\mathbf{i}(N-\ell+1)}(N-\ell) \cdot \prod_{\ell=1}^{j} u_{\mathbf{i}(j-\ell+1)}(j-\ell)\right)
$$

which holds in $R$ (at the $\ell$-th step of the process we multiply the preceding equality by $v_{\mathbf{i}(\ell)}(\ell-1)$ on the left and then apply $(41)$ with $\left.t=\ell\right)$. Similarly, we have that

$$
\left(\prod_{\ell=1}^{N} v_{\mathbf{i}(N-\ell+1)}\right) Q_{p(k)}^{s}=\sum_{j=1}^{N} \mu_{j, k}^{s} \cdot\left(\prod_{\ell=1}^{N-j} v_{\mathbf{i}(N-\ell+1)}(N-\ell) \cdot \prod_{\ell=1}^{j} u_{\mathbf{i}(j-\ell+1)}(j-\ell)\right) .
$$

We denote the left-hand sides of (42) and (43) by $\widetilde{P}_{p(k)}^{s}$ and $\widetilde{Q}_{p(k)}^{s}$, respectively, and set $\tilde{v}:=\prod_{\ell=1}^{N} v_{\mathbf{i}(N-\ell+1)}$. Note that $\tilde{v} \in S$. Then

$$
\tilde{v}^{-1} \widetilde{P}_{p(k)}^{s}=P_{p(k)}^{s}, \quad \tilde{v}^{-1} \widetilde{Q}_{p(k)}^{s}=Q_{p(k)}^{s} \quad\left(1 \leq k \leq N ; 1 \leq s \leq l^{\prime 2}\right) .
$$

Now (29) can be rewritten as

$$
\widetilde{Q}_{p(k)}^{s}\left(a_{p(k)}^{s}\right)^{-1} C_{p(k)}^{s}=\widetilde{P}_{p(k)}^{s} \quad\left(1 \leq k \leq N ; 1 \leq s \leq l^{\prime \prime}\right) .
$$

Choosing $\tilde{a}_{p(k)}^{s} \in S$ and $\tilde{q}_{p(k)}^{s} \in R$ such that

$$
\tilde{a}_{p(k)}^{s} \widetilde{Q}_{p(k)}^{s}=a_{p(k)}^{s} \tilde{q}_{p(k)}^{s} \quad\left(1 \leq k \leq N ; 1 \leq s \leq l^{\prime 2}\right)
$$

we can rewrite (45) as follows:

$$
\tilde{q}_{p(k)}^{s} C_{p(k)}^{s}=\tilde{a}_{p(k)}^{s} \widetilde{P}_{p(k)}^{s} \quad\left(1 \leq k \leq N ; 1 \leq s \leq l^{\prime 2}\right) .
$$

This relation holds in $R$. In view of (30) we have that

$$
Q_{p(k)}^{s}=Q_{2 ; p(k)}^{s}\left(Q_{1 ; p(k)}^{s}\right)^{-1} \quad\left(1 \leq k \leq N ; 1 \leq s \leq l^{\prime 2}\right) .
$$


Combining this with (44) we obtain

$$
\widetilde{Q}_{p(k)}^{s} Q_{1 ; p(k)}^{s}=\tilde{v} Q_{2 ; p(k)}^{s} \quad\left(1 \leq k \leq N ; 1 \leq s \leq l^{\prime 2}\right) .
$$

This relation holds in $R$ as well.

Finally, in view of (29) and (30) we can replace (11) by the following relation:

$$
e_{i j} w_{t}=w_{t} e_{i j} \quad\left(1 \leq i, j \leq l^{\prime} ; 1 \leq t \leq 2 d\right) .
$$

Since $v_{i}^{-1} u_{i}=w_{i}=u_{i}^{\prime} v_{i}^{\prime-1}$ for some $u_{i}^{\prime} \in R$ and $v_{i}^{\prime} \in S$, the latter can be rewritten as

$$
s_{i j}^{-1} E_{i j} v_{t}^{-1} u_{t}=u_{t}^{\prime} v_{t}^{-1} s_{i j}^{-1} E_{i j} \quad\left(1 \leq i, j \leq l^{\prime} ; 1 \leq t \leq 2 d\right) .
$$

There exists $v_{i j ; t}, b_{i j ; t} \in S$ and $E_{i j ; t}, D_{i j ; t} \in R$ such that

$$
\begin{aligned}
v_{i j ; t} E_{i j} & =E_{i j ; t} v_{t} ; \\
s_{i j} v_{t}^{\prime} D_{i j ; t} & =E_{i j} b_{i j ; t}
\end{aligned}
$$

for all $1 \leq i, j \leq l^{\prime}$ and $1 \leq t \leq 2 d$. Then (49) gives rise to the relations

$$
E_{i j ; t} u_{t} b_{i j ; t}=v_{i j ; t} s_{i j} u_{t}^{\prime} D_{i j ; t} \quad\left(1 \leq i, j \leq l^{\prime} ; 1 \leq t \leq 2 d\right)
$$

which hold in $R$.

We denote by $X$ the set of all elements

$$
\begin{aligned}
& E_{i j}, E_{i j}^{\prime}, C_{i j}^{k}, E_{i j, t k}, E_{i j, t h}^{k}, C_{i j, t h}^{k}, q_{k}, D_{i j}^{k}, T_{i j}^{k}, q_{p(j) ; k}, \\
& u_{i}, u_{i}^{\prime}, u_{i, j}, b_{i, j}^{\prime}, u_{i(1), \ldots, i(k)}, u_{\mathbf{i}(j)}(t), \tilde{q}_{p(k)}^{s}, E_{i j ; t}, D_{i j ; t}
\end{aligned}
$$

and by $Y$ the set of all elements

$$
\begin{aligned}
& s_{i j}, s_{i j}^{\prime}, a_{i j}^{k}, r_{i j, t k}, r_{i j, t h}^{k}, a_{i j, t h}^{k}, s_{1, \ldots, k}, s_{i j}^{k}, s_{i j ; k}, s_{p(1), \ldots, p(j) ; k}, \\
& Q_{1 ; p(k)}^{s}, Q_{2 ; p(k)}^{s}, v_{i}, v_{i}^{\prime}, v_{i, j}, b_{i, j}, v_{i(1), \ldots, i(k)}, v_{\mathbf{i}(j)}(t), \tilde{a}_{p(k)}^{s}, v_{i j ; t}, b_{i j ; t} .
\end{aligned}
$$

3.3. Let $X \subset R$ and $Y \subset S$ be the finite subsets introduced in 3.1 and 3.2. Obviously, they lie in $R_{m}$ for some $m \gg 0$, hence involve only finitely many scalars in $\mathbb{C}$. From now on we will always assume that those scalars are in $A$ and hence $X \cup Y \subset R_{A}$. It will be crucial for us in what follows to work with those admissible rings $A$ for which the images of the elements of $Y$ in $R_{\mathbb{k}}=\left(R_{A} / \mathfrak{P} R_{A}\right) \otimes_{A / \mathfrak{P}} \mathbb{k}$ remain regular for all maximal ideals $\mathfrak{P}$ of $A$. Our next result ensures that such admissible rings do exist.

Lemma 3.1. Let $s$ be a regular element of $R$ contained in $R_{A}$ and assume that $A$ satisfies the conditions imposed in 2.7. Then there exists an admissible extension $B$ of $A$ such that for every $\mathfrak{P} \in$ Specm $B$ the element $s \otimes 1$ is regular in $R_{B} \otimes_{B} \mathbb{k}_{\mathfrak{P}} \cong$ $\left(R_{B} / \mathfrak{P} R_{B}\right) \otimes_{B / \mathfrak{P}} \mathbb{k}$.

Proof. Since $s \cdot R_{A}$ is a right ideal of $R_{A}$, the graded $A$-module $\operatorname{gr}\left(s \cdot R_{A}\right)$ is an ideal of the commutative Noetherian ring $\operatorname{gr}\left(R_{A}\right)$. Hence $\operatorname{gr}\left(s \cdot R_{A}\right)$ is a finitely generated $\operatorname{gr}\left(R_{A}\right)$-module. As $A$ is a Noetherian domain, applying [10, Thm. 14.4] shows that there is a nonzero $a_{1} \in A$ such that each $\left(\operatorname{gr}\left(s \cdot R_{A}\right)(n)\right)\left[a_{1}^{-1}\right]$ is a free $A\left[a_{1}^{-1}\right]$-module of finite rank. Since $\left(\operatorname{gr}\left(s \cdot R_{A}\right)(n)\right)\left[a_{1}^{-1}\right] \cong\left(\operatorname{gr}\left(s \cdot R_{A\left[a_{1}^{-1}\right]}\right)\right)(n)$ for all $n$, we see that there exists an admissible ring $\tilde{A} \subset \mathbb{C}$ containing $A$ such that all graded components of $\operatorname{gr}\left(s \cdot R_{\tilde{A}}\right)$ are free $\tilde{A}$-modules of finite rank. Since we can repeat this argument with the left ideal $R_{A} \cdot s$ in place of $s \cdot R_{A}$, it can be assumed, after enlarging $\tilde{A}$ possibly, that all graded components of $\operatorname{gr}\left(R_{\tilde{A}} \cdot s\right)$ are free $\tilde{A}$-modules of finite rank as well. 
Since $\operatorname{gr}\left(R_{A}\right)$ is a finitely generated $A$-algebra, we can also apply [10, Thm. 14.4] to the graded $\operatorname{gr}\left(R_{A}\right)$-module $\operatorname{gr}\left(R_{A} / s \cdot R_{A}\right) \cong \operatorname{gr}\left(R_{A}\right) / \operatorname{gr}\left(s \cdot R_{A}\right)$ to deduce that there is a nonzero $a_{2} \in A$ such that all graded components of

$$
\operatorname{gr}\left(R_{A} / s \cdot R_{A}\right)\left[a_{2}^{-1}\right] \cong\left(\operatorname{gr}\left(R_{A}\right) / \operatorname{gr}\left(s \cdot R_{A}\right)\right)\left[a_{2}^{-1}\right] \cong \operatorname{gr}\left(R_{A\left[a_{2}^{-1}\right]}\right) / \operatorname{gr}\left(s \cdot R_{A\left[a_{2}^{-1}\right]}\right)
$$

are free $A\left[a_{2}^{-1}\right]$-modules of finite rank. Replacing $s \cdot R_{A}$ by $R_{A} \cdot s$ in this argument we observe that the same applies to all graded components of $\operatorname{gr}\left(R_{A\left[a_{3}^{-1}\right]}\right) / \operatorname{gr}\left(R_{A\left[a_{3}^{-1}\right]} \cdot s\right)$ for a suitable nonzero $a_{3} \in A$.

We conclude that there exists an admissible extension $B$ of $A$ such that all graded components of $\operatorname{gr}\left(s \cdot R_{B}\right), \operatorname{gr}\left(R_{B} \cdot s\right), \operatorname{gr}\left(R_{B}\right) / \operatorname{gr}\left(s \cdot R_{B}\right)$ and $\operatorname{gr}\left(R_{B}\right) / \operatorname{gr}\left(R_{B} \cdot s\right)$ are free $B$-modules of finite rank. Straightforward induction on filtration degree now shows that the free $B$-modules $s \cdot R_{B} \cong R_{B}$ and $R_{B} \cdot s \cong R_{B}$ are direct summands of $R_{B}$. Let $R_{B}^{\prime}$ and $R_{B}^{\prime \prime}$ be $B$-submodules of $R_{B}$ such that $R_{B}=\left(s \cdot R_{B}\right) \oplus R_{B}^{\prime}$ and $R_{B}=\left(R_{B} \cdot s\right) \oplus R_{B}^{\prime \prime}$

We now take any maximal ideal $\mathfrak{P}$ of $B$, denote by $\mathfrak{f}$ the finite field $B / \mathfrak{P}$, and write $\bar{x}$ for the image of $x \in R_{B}$ in $R_{\mathbb{k}}=\left(R_{B} / \mathfrak{P} R_{B}\right) \otimes_{\mathfrak{f}} \mathbb{k}$. Note that $R_{\mathfrak{f}}:=R_{B} / \mathfrak{P} R_{B}$ is an $\mathfrak{f}$-form of the $\mathbb{k}$-vector space $R_{\mathbb{k}}$. Suppose $\bar{s} \cdot \bar{u}=0$ for some $u \in R_{B}$. Then

$$
\begin{aligned}
s \cdot u & \in\left(s \cdot R_{B}\right) \cap \mathfrak{P} R_{B}=\left(s \cdot R_{B}\right) \cap\left(\mathfrak{P}\left(s \cdot R_{B}\right) \oplus \mathfrak{P} R_{B}^{\prime}\right) \\
& \left.=\left(s \cdot R_{B}\right) \cap\left(s \cdot \mathfrak{P} R_{B}\right) \oplus \mathfrak{P} R_{B}^{\prime}\right)=s \cdot \mathfrak{P} R_{B} .
\end{aligned}
$$

Therefore, $s \cdot u=s \cdot u^{\prime}$ for some $u^{\prime} \in \mathfrak{P} R_{B}$. Since $s$ is a regular element of $R$ and $s \cdot\left(u-u^{\prime}\right)=0$, we deduce that $u=u^{\prime} \in \mathfrak{P} R_{B}$. This yields $\bar{u}=0$. If $\bar{v} \cdot \bar{s}=0$ for some $v \in R_{B}$, then we use the decomposition $R_{B}=\left(R_{B} \cdot s\right) \oplus R_{B}^{\prime \prime}$ and argue as before to deduce that $\bar{v}=0$. Hence $\bar{s}$ is a regular element of $R_{\mathrm{f}}$.

Let $l_{\bar{s}}: R_{\mathbb{k}} \rightarrow R_{\mathbb{k}}$ and $r_{\bar{s}}: R_{\mathbb{k}} \rightarrow R_{\mathbb{k}}$ denote the left and right multiplication by $\bar{s}$, respectively. Denote by $\left(R_{\mathbb{k}}\right)_{j}$ the $j$ th component of the filtration of $R_{\mathbb{k}}$ induced by the canonical filtration of $U\left(\mathfrak{g}_{\mathbb{k}}\right)$ and set $\left(R_{\mathfrak{f}}\right)_{j}:=\left(R_{\mathbb{k}}\right)_{j} \cap R_{\mathfrak{f}}$. We know that $\bar{s} \in\left(R_{\mathfrak{f}}\right)_{\ell}$ for some $\ell$, whereas the regularity of $\bar{s}$ in $R_{\mathfrak{f}}$ yields that the $\mathfrak{f}$-linear maps $l_{\bar{s}}:\left(R_{\mathfrak{f}}\right)_{j} \rightarrow\left(R_{\mathfrak{f}}\right)_{j+\ell}$ and $r_{\bar{s}}:\left(R_{\mathfrak{f}}\right)_{j} \rightarrow\left(R_{\mathfrak{f}}\right)_{j+\ell}$ are injective for all $j \in \mathbb{Z}_{+}$. Standard linear algebra then shows that so are all $\mathbb{k}$-linear maps $l_{\bar{s}}:\left(R_{\mathbb{k}}\right)_{j} \rightarrow\left(R_{\mathbb{k}}\right)_{j+\ell}$ and $r_{\bar{s}}:\left(R_{\mathbb{k}}\right)_{j} \rightarrow\left(R_{\mathbb{k}}\right)_{j+\ell}$. In other words, $\bar{s}$ is regular in $R_{\mathbb{k}}$ as claimed.

\section{Proving the main results}

4.1. From now on we assume that for every $s \in Y$ the element $s \otimes 1$ is regular in $R_{\mathbb{k}}=\left(R_{A} / \mathfrak{P} R_{A}\right) \otimes_{\mathfrak{f}} \mathbb{k}$ for every $\mathfrak{P} \in \operatorname{Specm} A$ (here $\mathfrak{f}=A / \mathfrak{P}$ ). Since $Y$ is a finite set, this is a valid assumption thanks to Lemma 3.1. We also assume that our admissible ring $A$ satisfies all requirements mentioned in Sect. 2. The discussion in 2.8 then shows that the simple algebraic group $G_{\mathbb{k}}$ acts on $R_{\mathbb{k}}$ as algebra automorphisms and preserves the filtration of $R_{\mathbb{k}}$ induced by the canonical filtration of $U\left(\mathfrak{g}_{\mathbb{k}}\right)$.

Since $U\left(\mathfrak{g}_{\mathbb{k}}\right)$ is a finite module over its centre, so is its homomorphic image $R_{\mathbb{k}}=$ $\left(U\left(\mathfrak{g}_{A}\right) / \mathcal{J}_{A}\right) \otimes_{\mathfrak{f}} \mathbb{k} \cong U\left(\mathfrak{g}_{\mathbb{k}}\right) / \mathcal{J}_{\mathbb{k}}$. Being a homomorphic image of $U\left(\mathfrak{g}_{\mathbb{k}}\right)$, the ring $R_{\mathbb{k}}$ is Noetherian and, moreover, an affine PI-algebra over $\mathbb{k}$. Let $I_{1}, \ldots, I_{\nu}$ be the minimal primes of $R_{\mathbb{k}}$ and $N_{\mathbb{k}}:=\bigcap_{j=1}^{\nu} I_{j}$. Then $\nu=\nu(\mathfrak{P}) \in \mathbb{N}$ and $N_{\mathbb{k}}$ is the maximal nilpotent ideal of $R_{\mathbb{k}}$; see [33, Thm. 2]. In particular, $\bar{R}_{\mathbb{k}}:=R_{\mathbb{k}} / N_{\mathbb{k}}$ is a semiprime 
Noetherian ring. By Goldie's theory, the set $\bar{S}_{\mathbb{k}}$ of all regular elements of $\bar{R}_{\mathbb{k}}$ is an Ore set in $\bar{R}_{\mathbb{k}}$ and the quotient ring $Q\left(\bar{R}_{\mathbb{k}}\right)=\bar{S}_{\mathbb{k}}^{-1} \bar{R}_{\mathbb{k}}$ is semisimple and Artinian.

Write $Z\left(\bar{R}_{\mathbb{k}}\right)$ for the centre of $\bar{R}_{\mathbb{k}}$ and $\mathcal{C}\left(Z\left(\bar{R}_{\mathbb{k}}\right)\right)$ for the set of all elements of $Z\left(\bar{R}_{\mathbb{k}}\right)$ which are regular in $\bar{R}_{\mathbb{k}}$. Since $\bar{R}_{\mathbb{k}}$ is a finite module over the image of the $p$-centre of $U\left(\mathfrak{g}_{\mathbb{k}}\right)$ in $\bar{R}_{\mathbb{k}}$, it is algebraic over $Z\left(\bar{R}_{\mathbb{k}}\right)$. Applying [1, Thm. 2] now yields that $\mathcal{Q}\left(\bar{R}_{\mathbb{k}}\right)$ is obtained from $\bar{R}_{\mathbb{k}}$ by inverting the elements from $\mathrm{C}\left(Z\left(\bar{R}_{\mathbb{k}}\right)\right.$ ) (the latter is obviously an Ore set in $\bar{R}_{\mathbb{k}}$ ).

Proposition 4.1. There exists a unital subalgebra $\mathfrak{C}_{\mathbb{k}}$ of $\mathcal{Q}\left(\bar{R}_{\mathbb{k}}\right)$ isomorphic to $\operatorname{Mat}_{l^{\prime}}(\mathbb{k})$ and such that $Q\left(\bar{R}_{\mathbb{k}}\right) \cong \mathfrak{C}_{\mathbb{k}} \otimes \mathfrak{D}_{\mathbb{k}}$ where $\mathfrak{D}_{\mathbb{k}}$ is the centraliser of $\mathfrak{C}_{\mathbb{k}}$ in $\mathcal{Q}\left(\bar{R}_{\mathbb{k}}\right)$.

Proof. The ring theoretic notation used below will follow that of [22]. Given a twosided ideal $I$ of the ring $R_{\mathbb{k}}$ we write $\mathcal{C}^{\prime}(I)$ for the set of all elements $r \in R_{\mathbb{k}}$ for which the coset $r+I$ is left regular in the ring $R_{\mathbb{k}} / I$ (the latter means that $r \cdot x \in I$ for $x \in R_{\mathbb{k}}$ implies $\left.x \in I\right)$. We denote by $\mathrm{C}(I)$ the set of all elements $r \in R_{\mathbb{k}}$ such that the coset $r+I$ is left and right regular in $R_{\mathbb{k}} / I$. As we know, for each $y \in Y$ the element $y \otimes 1$ is regular in $R_{\mathbb{k}}$. In particular, $y \otimes 1 \in \mathrm{C}^{\prime}(0)$. To ease notation we now let $\bar{x}$ denote the image of $x \in R_{A}$ in $\bar{R}_{\mathbb{k}}=R_{\mathbb{k}} / N_{\mathbb{k}}$. As the ring $R_{\mathbb{k}}$ is right Noetherian, it follows from $[14,2.3,2.5]$ that $\mathrm{C}^{\prime}(0) \subseteq \mathrm{C}\left(N_{\mathbb{k}}\right)$ (see also [22, Prop. 4.1.3(iii)]). This shows that for every $y \in Y$ the element $\bar{y}$ is regular in $\bar{R}_{\mathbb{k}}$.

The subset $\bar{X} \cup \bar{Y}$ of $\bar{R}_{\mathbb{k}}$ contains elements satisfying the relations (9), (12), (13), (14), (15), (16), (19), (20), (22), (25), (26). Since all elements of $\bar{Y}$ involved in these relations remain regular in $\bar{R}_{\mathbb{k}}$ and each step of the procedure described in 3.1 is reversible, we can find elements $\bar{e}_{i j}$ and $\bar{c}_{i j}^{k}$ in $\mathcal{Q}\left(\bar{R}_{\mathbb{k}}\right)$, where $1 \leq i, j \leq l^{\prime}$ and $1 \leq k \leq n$, satisfying the relations $(6),(7),(10),(11)$. We denote by $\overline{\mathfrak{C}}_{\mathbb{k}}$ the $\mathbb{k}$-span of the $\bar{e}_{i j}$ 's. Thanks to (6) and (7), it is a homomorphic image of $\mathrm{Mat}_{l^{\prime}}(\mathbb{k})$ and a unital subalgebra of $\mathcal{Q}\left(\bar{R}_{\mathbb{k}}\right)$. Therefore, $\mathfrak{C}_{\mathbb{k}} \cong \operatorname{Mat}_{l^{\prime}}(\mathbb{k})$ as $\mathbb{k}$-algebras.

In view of (11) all elements $\bar{c}_{i j}^{k}$ commute with $\mathfrak{C}_{\mathbb{k}}$, whilst (10) implies that the $\bar{g}_{k}$ 's lie in $\mathfrak{C}_{\mathbb{k}} \cdot \mathfrak{D}_{\mathbb{k}}$ where $\mathfrak{D}_{\mathbb{k}}$ is the centraliser of $\mathfrak{C}_{\mathbb{k}}$ in $\mathcal{Q}\left(\bar{R}_{\mathbb{k}}\right)$. As the inverses of the elements from $\mathcal{C}\left(Z\left(\bar{R}_{\mathbb{k}}\right)\right)$ lie in $\mathfrak{D}_{\mathbb{k}}$ as well and $\mathcal{Q}\left(\bar{R}_{\mathbb{k}}\right)=\bar{S}_{\mathbb{k}}^{-1} \bar{R}_{\mathbb{k}}=\left(\mathcal{C}\left(Z\left(\bar{R}_{\mathbb{k}}\right)\right)\right)^{-1} \bar{R}_{\mathbb{k}}$ by our earlier remarks, we deduce that $\mathcal{Q}\left(\bar{R}_{\mathbb{k}}\right)=\mathfrak{C}_{\mathbb{k}} \cdot \mathfrak{D}_{\mathbb{k}}$. As a consequence, there exists a surjective algebra homomorphism $\psi: \mathfrak{C}_{\mathbb{k}} \otimes \mathfrak{D}_{\mathbb{k}} \rightarrow \mathcal{Q}\left(\bar{R}_{\mathbb{k}}\right)$. Since $\mathfrak{C}_{\mathbb{k}}$ is a matrix algebra, it is straightforward to see that $\psi$ is injective. This completes the proof.

4.2. Let $Z\left(\bar{R}_{\mathbb{k}}\right)$ be the centre of $\bar{R}_{\mathbb{k}}$ and denote by $Z_{p}\left(\bar{R}_{\mathbb{k}}\right)$ the image of the $p$-centre $Z_{p}\left(\mathfrak{g}_{\mathrm{k}}\right)$ in $\bar{R}_{\mathbb{k}}$. Recall from (5) that the commutative $A$-algebra $\operatorname{gr}\left(R_{A}\right)$ is generated by $D$ homogeneous elements as a module over its graded polynomial subalgebra $A\left[y_{1}, \ldots, y_{2 d}\right]$, where $d=d(e)$.

Lemma 4.1. There exists a $\mathbb{k}$-subalgebra $\bar{Z}_{0}$ of $Z_{p}\left(\bar{R}_{\mathbb{k}}\right)$ generated by $2 d$ elements and such that $\bar{R}_{\mathbb{k}}$ is generated as a $\bar{Z}_{0}$-module by $D p^{2 d}$ elements.

Proof. We follow the proof of [29, Lemma 3.2] very closely. Write $\left(R_{A}\right)_{j}\left(\operatorname{resp} .\left(R_{\mathbb{k}}\right)_{j}\right)$ for the image in $R_{A}$ (resp. $R_{\mathbb{k}}$ ) of the $j$ th component of the canonical filtration of $U\left(\mathfrak{g}_{A}\right)$ (resp. $\left.U\left(\mathfrak{g}_{\mathbb{k}}\right)\right)$.

Suppose that $y_{i}$ has degree $a_{i}$, where $1 \leq i \leq 2 d$, and $v_{k}$ has degree $l_{k}$, where $1 \leq k \leq D$, and let $\Phi_{A}: S\left(\mathfrak{g}_{A}\right) \rightarrow \operatorname{gr}\left(R_{A}\right)$ denote the canonical homomorphism. For $1 \leq i \leq 2 d$ (resp. $1 \leq k \leq D)$ choose $u_{i} \in U\left(\mathfrak{g}_{A}\right)$ (resp. $w_{k} \in U_{l_{k}}\left(\mathfrak{g}_{A}\right)$ ) such that 
$\Phi_{A}\left(\operatorname{gr}_{a_{i}} u_{i}\right)=y_{i}\left(\operatorname{resp} . \Phi_{A}\left(\operatorname{gr}_{l_{k}} w_{k}\right)=v_{k}\right)$. Let $\bar{u}_{i}\left(\right.$ resp. $\left.\bar{w}_{k}\right)$ denote the image of $u_{i}$ (resp. $w_{k}$ ) in $R_{\mathbb{k}}=\left(U\left(\mathfrak{g}_{A}\right) / \mathcal{J}_{A}\right) \otimes_{A} \mathbb{k}_{\mathfrak{P}}$. For every $n \in \mathbb{Z}_{+}$the set

$$
\left\{w_{k} u_{1}^{i_{1}} \cdots u_{2 d}^{i_{2 d}} \mid l_{k}+\sum_{j=1}^{2 d} i_{j} a_{j} \leq n ; 1 \leq k \leq D\right\}
$$

spans the $A$-module $\left(R_{A}\right)_{n}$. In view of our earlier remarks this implies that the set

$$
\left\{\bar{w}_{k} \bar{u}_{1}^{i_{1}} \cdots \bar{u}_{2 d}^{i_{2 d}} \mid l_{k}+\sum_{j=1}^{2 d} i_{j} a_{j} \leq n ; 1 \leq k \leq D\right\}
$$

spans the $\mathbb{k}$-space $\left(R_{\mathbb{k}}\right)_{n}$. Since $\operatorname{gr}_{p a_{i}}\left(\bar{u}_{i}^{p}\right)=\left(\operatorname{gr}_{a_{i}} \bar{u}_{i}\right)^{p}$ is a $p$ th power in $S\left(\mathfrak{g}_{\mathbb{k}}\right)$, for every $i \leq 2 d$ there exists a $z_{i} \in Z_{p}\left(\mathfrak{g}_{\mathbb{k}}\right) \cap U_{a_{i}}\left(\mathfrak{g}_{\mathrm{k}}\right)$ such that $\bar{u}_{i}^{p}-z_{i} \in U_{p a_{i}-1}\left(\mathfrak{g}_{\mathbb{k}}\right)$. We let $Z_{0}$ be the $\mathbb{k}$-subalgebra of $Z_{p}\left(\mathfrak{g}_{\mathbb{k}}\right)$ generated by $z_{1}, \ldots, z_{2 d}$ and denote by $\bar{Z}_{0}$ the image of $Z_{0}$ in $\bar{R}_{\mathbb{k}}=R_{\mathbb{k}} / N_{\mathbb{k}}$.

Let $R_{\mathbb{k}}^{\prime}$ the $Z_{0}$-submodule of $R_{\mathbb{k}}$ generated by all $\bar{w}_{k} \bar{u}_{1}^{i_{1}} \cdots \bar{u}_{2 d}^{i_{2 d}}$ with $0 \leq i_{j} \leq p-1$ and $1 \leq k \leq D$. Using the preceding remarks and induction on $n$ we now obtain that $\left(R_{\mathbb{k}}\right)_{n} \subset R_{\mathbb{k}}^{\prime}$ for all $n \in \mathbb{Z}_{+}$. But then $R_{\mathbb{k}}=R_{\mathbb{k}}^{\prime}$, implying that the set

$$
\Lambda:=\left\{\bar{w}_{k} \bar{u}_{1}^{i_{1}} \cdots \bar{u}_{2 d}^{i_{2 d}} \mid 0 \leq i_{j} \leq p-1 ; 1 \leq k \leq D\right\}
$$

generates $R_{\mathbb{k}}$ as an $Z_{0}$-module. Obviously, $|\Lambda| \leq D p^{2 d}$. As $\bar{R}_{\mathbb{k}}$ is a homomorphic image of $R_{\mathbb{k}}$ and the action of $\bar{Z}_{0}$ on $\bar{R}_{\mathbb{k}}$ is induced by that of $Z_{0} \subset Z_{p}\left(\mathfrak{g}_{\mathbb{k}}\right)$, the result follows.

Corollary 4.1. Every irreducible $\bar{R}_{\mathbb{k}}$-module has dimension $\leq \sqrt{D} \cdot p^{d}$.

Proof. This is an immediate consequence of Lemma 4.1, because the central elements of $\bar{R}_{\mathbb{k}}$ act on any irreducible $\bar{R}_{\mathbb{k}}$-module $V$ as scalar operators and the image of $\Lambda$ in End $V$ spans End $V$.

Proposition 4.2. The centre $Z\left(\bar{R}_{\mathbb{k}}\right)$ is an affine algebra over $\mathbb{k}$ and

$$
\operatorname{dim} Z\left(\bar{R}_{\mathbb{k}}\right)=\operatorname{dim} Z_{p}\left(\bar{R}_{\mathbb{k}}\right)=2 d .
$$

Proof. By Lemma $4.1, \bar{R}_{\mathbb{k}}$ is a finitely generated $\bar{Z}_{0}$-module. Since $\bar{Z}_{0}$ is an affine $\mathbb{k}$ algebra, $\bar{R}_{\mathbb{k}}$ is a Noetherian $\bar{Z}_{0}$-module. But then $Z\left(\bar{R}_{\mathbb{k}}\right)$ and $Z_{p}\left(\bar{R}_{\mathbb{k}}\right)$ are finitely generated $\bar{Z}_{0}$-modules. From this it is follows that the $\mathbb{k}$-algebra $Z\left(\bar{R}_{\mathbb{k}}\right)$ is affine (of course, the same is true for $Z_{p}\left(\bar{R}_{\mathbb{k}}\right)$, as it is a homomorphic image of $\left.Z_{p}\left(\mathfrak{g}_{\mathbb{k}}\right)\right)$. Both $Z\left(\bar{R}_{\mathbb{k}}\right)$ and $Z_{p}\left(\bar{R}_{\mathbb{k}}\right)$ being integral over $\bar{Z}_{0}$, the inclusions $\bar{Z}_{0} \hookrightarrow Z_{p}\left(\bar{R}_{\mathbb{k}}\right)$ and $\bar{Z}_{0} \hookrightarrow Z\left(\bar{R}_{\mathbb{k}}\right)$ give rise to finite morphisms Specm $\bar{Z}_{0} \rightarrow \operatorname{Specm} Z_{p}\left(\bar{R}_{\mathbb{k}}\right)$ and $\operatorname{Specm} \bar{Z}_{0} \rightarrow \operatorname{Specm} Z\left(\bar{R}_{\mathbb{k}}\right)$. Since $\bar{Z}_{0}$ is a homomorphic image of the polynomial algebra $\mathbb{k}\left[X_{1}, \ldots, X_{2 d}\right]$, we now obtain

$$
\operatorname{dim} Z\left(\bar{R}_{\mathbb{k}}\right)=\operatorname{dim} Z_{p}\left(\bar{R}_{\mathbb{k}}\right)=\operatorname{dim} \bar{Z}_{0} \leq 2 d .
$$

Recall from 4.1 that the simple algebraic group $G_{\mathbb{k}}$ acts rationally on $\bar{R}_{\mathbb{k}}$. Moreover, the canonical homomorphism $c: U\left(\mathfrak{g}_{\mathbb{k}}\right) \rightarrow R_{\mathbb{k}}=U\left(\mathfrak{g}_{\mathbb{k}}\right) / \mathcal{J}_{\mathbb{k}}$ is $G_{\mathbb{k}^{-}}$-equivariant. Since the inverse image under $c$ of the unique maximal nilpotent ideal $N_{\mathbb{k}}$ of $R_{\mathbb{k}}$ is $G_{\mathbb{k}^{-}}$ stable, both $Z_{p}\left(\bar{R}_{\mathbb{k}}\right) \cong Z_{p}\left(\mathfrak{g}_{\mathbb{k}}\right) /\left(Z_{p}\left(\mathfrak{g}_{\mathbb{k}}\right) \cap c^{-1}\left(N_{\mathbb{k}}\right)\right)$ and $Z\left(\bar{R}_{\mathbb{k}}\right)$ are stable under the action of $G_{\mathbb{k}}$ on $\bar{R}_{\mathbb{k}}$. Since $Z_{p}\left(\bar{R}_{\mathbb{k}}\right)$ is a homomorphic image of $Z_{p}\left(\mathfrak{g}_{\mathbb{k}}\right)$, the maximal spectrum $\mathcal{V}_{\mathfrak{P}}(M):=\operatorname{Specm} Z_{p}\left(\bar{R}_{\mathbb{k}}\right)$ identifies with a Zariski closed subset of $\mathfrak{g}_{\mathbb{k}}^{*}$ (see 2.4 for more detail). By our discussion in 2.6, the affine $G_{\mathbb{k}^{\mathrm{B}}}$-variety $\mathcal{V}_{\mathfrak{P}}(M)$ contains a linear function $\Psi \in \chi+\mathfrak{m}_{\mathbb{k}}^{\perp}$. Indeed, it is immediate from the definition of $R_{A}$ that $\widetilde{M}_{\mathbb{k}}=\widetilde{M}_{A} \otimes_{A} \mathbb{k}$ is an $R_{\mathbb{k}}$-module. Therefore, $\bar{R}_{\mathbb{k}}=R_{\mathbb{k}} / N_{\mathbb{k}}$ acts on its simple 
quotient $\widetilde{M}_{\mathbb{k}, \Psi}$. Since the $x^{p}-x^{[p]}$ acts on $\widetilde{M}_{\mathbb{k}, \Psi}$ as $\Psi(x)^{p}$ Id for every $x \in \mathfrak{g}_{\mathbb{k}}$ and $Z_{p}\left(\mathfrak{g}_{\mathbb{k}}\right) \cap c^{-1}\left(N_{\mathbb{k}}\right)$ annihilates $\widetilde{M}_{\mathbb{k}, \Psi}$, we see that $\Psi$ induces an algebra homomorphism $Z_{p}\left(\bar{R}_{\mathbb{k}}\right) \rightarrow \mathbb{k}$. In other words, $\Psi \in \mathcal{V}_{\mathfrak{P}}(M)$.

Given $j \in \mathbb{Z}^{+}$we define $\Xi_{j}:=\left\{\eta \in \mathfrak{g}_{\mathbb{k}}^{*} \mid \operatorname{dim} \mathfrak{z} \xi \leq 2 j\right\}$, a Zariski closed, conical subset of $\mathfrak{g}_{\mathbb{k}}^{*}$. There is a cocharacter $\lambda: \mathbb{k}^{\times} \rightarrow G_{\mathbb{k}}$ such that $(\operatorname{Ad} \lambda(t))(x)=t^{i} x$ for all $x \in \mathfrak{g}_{k}(i)$ and $t \in \mathbb{k}^{\times}$. Let $\rho_{e}: \mathbb{k}^{\times} \rightarrow \mathrm{GL}\left(\mathfrak{g}_{\mathbb{k}}^{*}\right)$ denote the composition of $\mathrm{Ad}^{*} \lambda$ with the scalar cocharacter $\xi \mapsto t^{-2} \xi$, where $\xi \in \mathfrak{g}_{\mathbb{k}}^{*}$ and $t \in \mathbb{k}^{\times}$. Obviously, $\rho_{e}$ induces a contracting $\mathbb{k}^{\times}$-action on $\chi+\mathfrak{m}_{\mathbb{k}}^{\perp}$ with centre at $\chi$. Since for any $j$ the Zariski closed set $\left(\chi+\mathfrak{m}_{\mathbb{k}}^{\perp}\right) \cap \Xi_{j}$ is $\rho_{e}\left(\mathbb{k}^{\times}\right)$-stable and $\operatorname{dim} \mathfrak{z} \chi=2 d$, we see that $\left(\chi+\mathfrak{m}_{\mathrm{k}}^{\perp}\right) \cap \Xi_{j}=\emptyset$ for all $j<2 d$. This implies that $\operatorname{dim} \mathfrak{z} \Psi \geq 2 d$.

Since $\left(\mathrm{Ad}^{*} G_{\mathbb{k}}\right) \Psi \subset \mathcal{V}_{\mathfrak{P}}(M)$, we now deduce that $\operatorname{dim} \mathcal{V}_{\mathfrak{P}}(M) \geq 2 d$. In conjunction with (53) this gives $\operatorname{dim} Z\left(\bar{R}_{\mathbb{k}}\right)=\operatorname{dim} Z_{p}\left(\bar{R}_{\mathbb{k}}\right)=2 d$, as stated.

Remark 4.1. It follows from the proof of Proposition 4.2 that $\operatorname{dim} \mathfrak{z}_{\Psi}=2 d$ and the orbit $\left(\mathrm{Ad}^{*} G_{\mathbb{k}}\right) \Psi$ is open in the variety $\mathcal{V}_{\mathfrak{P}}(M)$. Moreover, arguing as in $[31,3.6]$ it is easy to observe that $\chi$ and $\Psi$ belong to the same sheet of $\mathfrak{g}_{\mathbb{k}}^{*}$.

4.3. In this subsection we assume that $\mathcal{D}_{M}$ is a Weyl skew-field and we adopt the notation and conventions of 4.1. By Proposition 4.1, there is a unital subalgebra $\mathfrak{C}_{\mathbb{k}} \cong \operatorname{Mat}_{l^{\prime}}(\mathbb{k})$ of $\mathcal{Q}\left(\bar{R}_{\mathbb{k}}\right)$ such that $\mathcal{Q}\left(\bar{R}_{\mathbb{k}}\right) \cong \mathfrak{C}_{\mathbb{k}} \otimes \mathfrak{D}_{\mathbb{k}}$ where $\mathfrak{D}_{\mathbb{k}}$ is the centraliser of $\mathfrak{C}_{\mathbb{k}}$ in $\mathcal{Q}\left(\bar{R}_{\mathbb{k}}\right)$.

Proposition 4.3. Suppose $\mathcal{D}_{M} \cong \mathcal{Q}\left(\mathbf{A}_{d}(\mathbb{C})\right)$ and the admissible ring $A$ satisfies all the requirements of 4.1. Then the $\mathbb{k}$-algebra $\mathfrak{D}_{\mathbb{k}}$ is isomorphic to the ring of fractions $\mathcal{Q}\left(\mathbf{A}_{d}(\mathbb{k})\right)$ and $\mathcal{Q}\left(\bar{R}_{\mathbb{k}}\right) \cong \operatorname{Mat}_{l^{\prime}}\left(\mathcal{Q}\left(\mathbf{A}_{d}(\mathbb{k})\right)\right)$.

Proof. First recall from 4.1 that given $x \in R_{A}$ we write $\bar{x}$ for the image of $x \otimes 1$ in $\bar{R}_{\mathbb{k}}=\left(R_{A} \otimes_{A} \mathbb{k}_{\mathfrak{P}}\right) / N_{\mathbb{k}}$. Repeating the argument used at the beginning of the proof of Proposition 4.1 we observe that for every $y \in Y$ the element $\bar{y}$ is regular in $\bar{R}_{\mathbb{k}}$.

The subset $\bar{X} \cup \bar{Y}$ of $\bar{R}_{\mathbb{k}}$ contains elements satisfying the relations (34), (37), (38), (39), (40), (41), (42), (43), (44), (46), (47), (48), (50), (51), (52). Since all elements of $\bar{Y}$ involved in these relations are regular and each step of the procedure described in 3.2 is reversible, we can find elements $w_{1}, \ldots, w_{2 d}$ in $\mathcal{Q}\left(\bar{R}_{\mathbb{k}}\right)$ satisfying the relations (27) and (28). We denote by $\mathcal{D}_{\mathbb{k}}^{\prime}$ the $\mathbb{k}$-subalgebra of $\mathcal{Q}\left(\bar{R}_{\mathbb{k}}\right)$ generated by the $w_{i}$ 's. Clearly, $\mathcal{D}_{\mathbb{k}}^{\prime}$ is a homomorphic image of the Weyl algebra $\mathbf{A}_{d}(\mathbb{k})$.

By (49), we have the inclusion $\mathcal{D}_{\mathbb{k}}^{\prime} \subset \mathfrak{D}_{\mathbb{k}}$. Since the images of the $Q_{i ; p(k)}^{s}$ 's with $i=1,2$ are regular in $\bar{R}_{\mathbb{k}}$ and $\mathcal{Q}\left(\bar{R}_{\mathbb{k}}\right)=\left(\mathcal{C}\left(Z\left(\bar{R}_{\mathbb{k}}\right)\right)^{-1} \bar{R}_{\mathbb{k}}\right.$ by our earlier remarks, we can combine $(30),(29),(10)$ and $(11)$ with the equality $\mathcal{Q}\left(\bar{R}_{\mathbb{k}}\right)=\mathfrak{C}_{\mathbb{k}} \cdot \mathfrak{D}_{\mathbb{k}}$ to obtain

$$
\mathfrak{D}_{\mathbb{k}}=\left(\mathcal{C}\left(Z\left(\bar{R}_{\mathbb{k}}\right)\right)^{-1} \mathcal{D}_{\mathbb{k}}^{\prime}\right. \text {. }
$$

Since it follows from Proposition 4.1 that $\mathfrak{D}_{\mathbb{k}}$ is a semiprime ring, (54) yields that $\mathcal{D}_{\mathbb{k}}^{\prime}$ has no nonzero nilpotent ideals, i.e. the ring $\mathcal{D}_{\mathbb{k}}^{\prime}$ is semiprime, too.

Let $\mathcal{C}\left(Z\left(\mathcal{D}_{\mathbb{k}}^{\prime}\right)\right)$ denote the set of all regular elements of $\mathcal{D}_{\mathbb{k}}^{\prime}$ contained in the centre of $\mathcal{D}_{\mathbb{k}}^{\prime}$. It is immediate from (54) that $\mathcal{C}\left(Z\left(\mathcal{D}_{\mathbb{k}}^{\prime}\right)\right) \subseteq \mathcal{C}\left(Z\left(\mathfrak{D}_{\mathbb{k}}\right)\right)$. So $\mathcal{C}\left(Z\left(\mathcal{D}_{\mathbb{k}}^{\prime}\right)\right)$ is a multiplicative subset of regular elements of $\mathcal{Q}\left(\bar{R}_{\mathbb{k}}\right)$ satisfying the left and right Ore condition.

Being a homomorphic image of $\mathbf{A}_{d}(\mathbb{k})$ the $\mathbb{k}$-algebra $\mathcal{D}_{\mathbb{k}}^{\prime}$ is finitely generated as a module over its centre. As $\mathcal{D}_{\mathbb{k}}^{\prime}$ is a semiprime ring, applying [1, Thm. 2] yields that 
$\mathcal{Q}\left(\mathcal{D}_{\mathbb{k}}^{\prime}\right)$ is obtained from $\mathcal{D}_{\mathbb{k}}^{\prime}$ by inverting the elements from $\mathcal{C}\left(Z\left(\mathcal{D}_{\mathbb{k}}^{\prime}\right)\right)$. Combining this with $(30)$ and $(29)$ we now deduce that $\bar{c}_{i j}^{k} \in\left(\mathcal{C}\left(Z\left(\mathcal{D}_{\mathbb{k}}^{\prime}\right)\right)\right)^{-1} \mathcal{D}_{\mathbb{k}}^{\prime}$ for all $1 \leq i, j \leq l^{\prime}$ and $1 \leq k \leq n$. But then (10) forces $\bar{g}_{k} \in\left(\mathcal{C}\left(Z\left(\mathcal{D}_{\mathbb{k}}^{\prime}\right)\right)\right)^{-1} \mathfrak{C}_{\mathbb{k}} \cdot \mathcal{D}_{\mathbb{k}}^{\prime}$ for all $1 \leq k \leq n$. This, in turn, yields that $\left(\mathcal{C}\left(Z\left(\mathcal{D}_{\mathbb{k}}^{\prime}\right)\right)\right)^{-1} \mathcal{D}_{\mathbb{k}}^{\prime}$ contains $\bar{R}_{\mathbb{k}}$ and hence $Z\left(\bar{R}_{\mathbb{k}}\right)$. Then our remarks earlier in the proof show that

$$
\mathfrak{D}_{\mathbb{k}}=\left(\mathcal{C}\left(Z\left(\mathcal{D}_{\mathbb{k}}^{\prime}\right)\right)\right)^{-1} \mathcal{D}_{\mathbb{k}}^{\prime}=\mathcal{Q}\left(\mathcal{D}_{\mathbb{k}}^{\prime}\right) .
$$

Let $Z_{d}(\mathbb{k})$ denote the centre of the Weyl algebra $\mathbf{A}_{d}(\mathbb{k})$. It is well known and easily seen that $\mathbf{A}_{d}(\mathbb{k})$ is a free $Z_{d}(\mathbb{k})$-module of rank $p^{2 d}$ and every two-sided ideal of $\mathbf{A}_{d}(\mathbb{k})$ is centrally generated. Furthermore, $Z_{d}(\mathbb{k})$ is a polynomial algebra in $2 d$ variables over $\mathbb{k}$. Since $\mathcal{D}_{\mathbb{k}}^{\prime}$ is a homomorphic image of $\mathbf{A}_{d}(\mathbb{k})$, its centre, $\bar{Z}_{d}$, is a homomorphic image of $Z_{d}(\mathbb{k})$. We let $\beta: Z_{d}(\mathbb{k}) \rightarrow \bar{Z}_{d}$ denote the corresponding homomorphism of $\mathbb{k}$-algebras.

Recall from 4.1 that $N_{\mathbb{k}}=\bigcap_{i=1}^{\nu} I_{i}$ where $I_{1}, \ldots, I_{\nu}$ are the minimal primes of $R_{\mathbb{k}}$. By the theory of semiprime Noetherian PI-algebras finite over their centres, all quotients $R_{\mathbb{k}} / I_{j}$ are prime and $\mathcal{Q}\left(\bar{R}_{\mathbb{k}}\right) \cong \mathcal{Q}\left(R_{\mathbb{k}} / I_{1}\right) \oplus \cdots \oplus \mathcal{Q}\left(R_{\mathbb{k}} / I_{\nu}\right)$ as $\mathbb{k}$-algebras. Moreover, each direct summand $\mathcal{Q}\left(R_{\mathbb{k}} / I_{j}\right)$ is a simple algebra finite dimensional over its centre $\mathcal{Q}\left(Z\left(R_{\mathbb{k}} / I_{j}\right)\right)$; see [32], [1]. In particular, this shows that $\mathcal{Q}\left(Z\left(\bar{R}_{\mathbb{k}}\right)\right)=$ $\left(\mathrm{C}\left(Z\left(\bar{R}_{\mathbb{k}}\right)\right)^{-1} Z\left(\bar{R}_{\mathbb{k}}\right)\right.$ injects into $\prod_{j=1}^{\nu} \mathcal{Q}\left(\bar{R}_{\mathbb{k}} / I_{j}\right)$, a direct product of fields.

On the other hand, the algebra $Z\left(\bar{R}_{\mathbb{k}}\right)$ being reduced, $\mathcal{Q}\left(Z\left(\bar{R}_{\mathbb{k}}\right)\right)$ itself is a direct product of fields. Furthermore, Proposition 4.2 implies that at least one of the fields involved as direct factors of $\mathcal{Q}\left(Z\left(\bar{R}_{\mathbb{k}}\right)\right)$ has transcendence degree over $\mathbb{k}$ equal to $2 d$. It follows that

$$
\text { tr. } \operatorname{deg}_{\mathbb{k}} Q\left(Z\left(R_{\mathbb{k}} / I_{\ell}\right)\right)=2 d \quad \text { for some } \ell \leq \nu .
$$

Since $\mathcal{Q}\left(\bar{R}_{\mathbb{k}}\right) \cong \mathfrak{C}_{\mathbb{k}} \otimes \mathcal{Q}\left(\mathcal{D}_{\mathbb{k}}^{\prime}\right)$, it follows from our discussion earlier in the proof that $\mathcal{Q}\left(Z\left(\bar{R}_{\mathbb{k}}\right)\right) \cong \mathcal{Q}\left(\bar{Z}_{d}\right)$ as $\mathbb{k}$-algebras. As the algebra $\bar{R}_{\mathbb{k}}$ is semiprime, its centre $Z\left(\bar{R}_{\mathbb{k}}\right)$ is reduced and hence the ring of fractions $Q\left(\bar{Z}_{d}\right)$ is a direct product of fields. If $\beta: Z_{d}(\mathbb{k}) \rightarrow \bar{Z}_{d}$ is not injective, then $\operatorname{dim} \bar{Z}_{d}<2 d$ and hence all fields involved as direct factors of $\mathcal{Q}\left(\bar{Z}_{d}\right)$ have transcendence degree over $\mathbb{k}$ less than $2 d$. Since this contradicts (56), the map $\beta$ must be injective. Then $\bar{Z}_{d} \cong Z_{d}(\mathbb{k})$, implying that $\mathcal{Q}\left(\mathfrak{D}_{\mathbb{k}}\right) \cong \mathcal{Q}\left(\mathbf{A}_{d}(\mathbb{k})\right)$ and $\mathcal{Q}\left(\bar{R}_{\mathbb{k}}\right) \cong \mathfrak{C}_{\mathbb{k}} \otimes \mathcal{Q}\left(\mathbf{A}_{d}(\mathbb{k})\right) \cong \operatorname{Mat}_{l^{\prime}}\left(\mathcal{Q}\left(\mathbf{A}_{d}(\mathbb{k})\right)\right)$, as claimed.

Corollary 4.2. If $\mathcal{D}_{M} \cong \mathcal{Q}\left(\mathbf{A}_{d}(\mathbb{C})\right)$ as $\mathbb{C}$-algebras and the admissible ring $A$ satisfies all the requirements of 4.1 , then $\bar{R}_{\mathbb{k}}$ is a prime ring.

Proof. Since $\mathcal{Q}\left(\bar{R}_{\mathbb{k}}\right)=\mathcal{C}\left(Z\left(\bar{R}_{\mathbb{k}}\right)\right)^{-1} \bar{R}_{\mathbb{k}}$ and the ring $\mathcal{Q}\left(\mathbf{A}_{d}(\mathbb{k})\right)$ is prime, this is an immediate consequence of Proposition 4.3.

Conjecture 4.1. We conjecture that under the above assumptions on $A$ the ring $\bar{R}_{\mathbb{k}}$ is prime for any finite dimensional simple Lie algebra $\mathfrak{g}$ and any primitive ideal $I=I_{M}$.

4.4. Write $\bar{I}_{j}$ for the image the minimal prime $I_{j}$ of $R_{\mathbb{k}}$ in $\bar{R}_{\mathbb{k}}=R_{\mathbb{k}} / N_{\mathbb{k}}$. Since each quotient $\bar{R}_{\mathbb{k}} / \bar{I}_{j}$ is a prime ring, its central subalgebra $Z_{p}\left(\bar{R}_{\mathbb{k}}\right) / \bar{I}_{j} \cap Z_{p}\left(\bar{R}_{\mathbb{k}}\right)$ is a domain. Since the PI-algebras module-finite over their Noetherian centres enjoy the lyingover, going-up and incomparability properties for prime ideals, every $\bar{I}_{j} \cap Z_{p}\left(\bar{R}_{\mathbb{k}}\right)$ is a minimal prime of $Z_{p}\left(\bar{R}_{\mathbb{k}}\right)$ and every minimal prime of $Z_{p}\left(\bar{R}_{\mathbb{k}}\right)$ is one of the $\bar{I}_{j} \cap Z_{p}\left(\bar{R}_{\mathbb{k}}\right)^{\text {'s; }}$; 
see [22, Ch. 10] for more detail. It follows that there is an $\ell \in\{1, \ldots, \nu\}$ such that $\operatorname{dim} Z_{p}\left(\bar{R}_{\mathbb{k}}\right)=\operatorname{dim} Z_{p}\left(\bar{R}_{\mathbb{k}}\right) / \bar{I}_{\ell} \cap Z_{p}\left(\bar{R}_{\mathbb{k}}\right)$. We now define $\mathcal{R}:=\bar{R}_{\mathbb{k}} / \bar{I}_{\ell}$ and $Z_{p}(\mathcal{R}):=$ $Z_{p}\left(\bar{R}_{\mathbb{k}}\right) / \bar{I}_{\ell} \cap Z_{p}\left(\bar{R}_{\mathbb{k}}\right)$. Then $\mathcal{R}$ is a prime Noetherian ring which is finitely generated as a $Z_{p}(\mathcal{R})$-module.

Since $G_{\mathbb{k}}$ is a connected group, every minimal prime $\bar{I}_{j}$ of $\bar{R}_{\mathbb{k}}$ is $G_{\mathbb{k}}$-stable. Therefore, $G_{\mathbb{k}}$ acts on $\mathcal{R}$ as algebra automorphisms. Recall from 4.2 the Zariski closed set $\mathcal{V}_{\mathfrak{P}}(M) \subset \mathfrak{g}_{\mathbb{k}}^{*}$ which we have identified with the maximal spectrum of $Z_{p}\left(\bar{R}_{\mathbb{k}}\right)$. As explained in the proof of Proposition 4.2, one of the components of $\mathcal{V}_{\mathfrak{P}}(M)$ contains a linear function $\Psi \in \chi+\mathfrak{m}_{\mathbb{k}}^{\perp}$ and $\operatorname{dim}\left(\operatorname{Ad}^{*} G\right) \Psi=2 d$.

By construction, the zero locus of $\bar{I}_{\ell} \cap Z_{p}\left(\bar{R}_{\mathbb{k}}\right)$ in $\mathcal{V}_{\mathfrak{P}}(M)$ is an irreducible component of maximal dimension in $\mathcal{V}_{\mathfrak{P}}(M)$. Since $\operatorname{dim} Z_{p}\left(\bar{R}_{\mathbb{k}}\right)=2 d$ by Proposition 4.2 and all irreducible components of $\mathcal{V}_{\mathfrak{P}}(M)$ are $G_{\mathbb{k}}$-stable, we see that $\Psi$, too, lies in an irreducible component of maximal dimension of $\mathcal{V}_{\mathfrak{P}}(M)$. But then the above discussion shows that we can choose $\ell \in\{1, \ldots, \nu\}$ such that the zero locus of $\bar{I}_{\ell} \cap Z_{p}\left(\bar{R}_{\mathbb{k}}\right)$ in $\mathcal{V}_{\mathfrak{P}}(M)$ coincides with the Zariski closure of $\left(\operatorname{Ad}^{*} G\right) \Psi$ in $\mathfrak{g}_{\mathfrak{k}}^{*}$. Therefore, no generality will be lost by assuming that $\left(\mathrm{Ad}^{*} G\right) \Psi$ is the unique open dense orbit of maximal spectrum $\operatorname{Specm} Z_{p}(\mathcal{R}) \subset \mathfrak{g}_{\mathbb{k}}^{*}$.

Since $\mathcal{R}$ is a Noetherian $Z_{p}(\mathcal{R})$-module, the centre $Z(\mathcal{R})$ is finitely generated and integral over $Z_{p}(\mathcal{R})$. Hence $Z_{p}(\mathcal{R})$ is an affine algebra over $\mathbb{k}$ and the morphism

$$
\mu: \operatorname{Specm} Z(\mathcal{R}) \rightarrow \operatorname{Specm} Z_{p}(\mathcal{R})
$$

induced by inclusion $Z_{p}(\mathcal{R}) \hookrightarrow Z(\mathcal{R})$ is finite. In particular, $\operatorname{dim} Z(\mathcal{R})=\operatorname{dim} Z_{p}(\mathcal{R})=$ $2 d$. As the ring $\mathcal{R}$ is prime, the centre $Z(\mathcal{R})$ is a domain and hence the affine variety $\mathcal{V}(\mathcal{R}):=\operatorname{Specm} Z(\mathcal{R})$ is irreducible. By our choice of $A$, the rational action of the group $G_{\mathbb{k}}$ on $U\left(\mathfrak{g}_{\mathbb{k}}\right)$ induces that on $Z(\mathcal{R})$. Thus, $\mathcal{V}(\mathcal{R})$ is an irreducible affine $G_{\mathbb{k}^{-}}$ variety.

Proposition 4.4. The following are true:

(i) The finite morphism $\mu: \mathcal{V}(\mathcal{R}) \rightarrow \operatorname{Specm} Z_{p}(\mathcal{R})$ is $G_{\mathbb{k}}$-equivariant and the inverse image of $\left(\operatorname{Ad}^{*} G\right) \Psi \subset \operatorname{Specm} Z_{p}(\mathcal{R})$ under $\mu$ is a unique open dense $G_{\mathbb{k}}$-orbit of $\mathcal{V}(\mathcal{R})$.

(ii) The stabiliser $\left(G_{\mathbb{k}}\right)_{c}=\left\{g \in G_{\mathbb{k}} \mid g \cdot c=c\right\}$ of any $c \in \mu^{-1}(\Psi)$ has the property that $Z_{G_{\mathbb{k}}}(\Psi)^{\circ} \subseteq\left(G_{\mathbb{k}}\right)_{c} \subseteq Z_{G_{\mathbb{k}}}(\Psi)$.

(iii) The coadjoint stabiliser $Z_{G_{\mathrm{k}}}(\Psi)$ acts transitively on the fibre $\mu^{-1}(\Psi)$.

Proof. It is clear from our earlier remarks that $\mu$ is a finite morphism equivariant under the action of $G_{\mathbb{k}}$. Let $\mathcal{V}(\mathcal{R})_{\text {reg }}$ denote the inverse image of $\left(\operatorname{Ad}^{*} G\right) \Psi$ under $\mu$. Since the map $\mu$ is $G_{\mathbb{k}}$-equivariant, we have that $\mathcal{V}(\mathcal{R})_{\operatorname{reg}}=\bigcup_{c \in \mu^{-1}(\Psi)} G_{\mathbb{k}} \cdot c$. As the morphism $\mu$ is finite, $\mu^{-1}(\Psi)$ is a finite set and $\operatorname{dim} \mathcal{V}(\mathcal{R})=2 d=\left(\operatorname{Ad}^{*} G\right) \Psi$. From this it is immediate that each orbit $G_{\mathbb{k}} \cdot c$ with $c \in \mu^{-1}(\Psi)$ is Zariski open in $\mathcal{V}(\mathcal{R})$. As the variety $\mathcal{V}(\mathcal{R})$ is irreducible, we see that $\left(G_{\mathbb{k}} \cdot c\right) \cap\left(G_{\mathbb{k}} \cdot c^{\prime}\right) \neq \emptyset$ for any two $c, c^{\prime} \in \mu^{-1}(\Psi)$. This forces $G_{\mathbb{k}} \cdot c=G_{\mathbb{k}} \cdot c^{\prime}$ for all $c, c^{\prime} \in \mu^{-1}(\Psi)$, implying that $\mu^{-1}\left(\mathcal{V}(\mathcal{R})_{\text {reg }}\right)=G_{\mathbb{k}} \cdot c$ for any $c \in \mu^{-1}(\Psi)$. This proves statement (i).

If $c \in \mu^{-1}(\Psi)$ and $g \in\left(G_{\mathbb{k}}\right)_{c}$, then

$$
\Psi=\mu(c)=\mu(g \cdot c)=\underset{24}{\left(\operatorname{Ad}^{*} g\right)} \mu(c)=\left(\operatorname{Ad}^{*} g\right) \Psi .
$$


Therefore, $\left(G_{\mathbb{k}}\right)_{c} \subseteq Z_{G_{\mathbb{k}}}(\Psi)$. On the other hand, the finite set $\mu^{-1}(c)$ is stable under the action of $Z_{G_{\mathrm{k}}}(\Psi)$. As $G_{\mathbb{k}}$ acts regularly on the affine algebraic variety $\mathcal{V}(\mathcal{R})$, it follows that the stabiliser $\left(G_{\mathbb{k}}\right)_{c}$ of any $c \in \mu^{-1}(\Psi)$ is a Zariski closed subgroup of finite index in $Z_{G_{\mathrm{k}}}(\Psi)$. So it must contain the connected component of identity in $Z_{G_{\mathrm{kk}}}(\Psi)$, and statement (ii) follows.

If $c, g(c) \in \mu^{-1}(\Psi)$ for some $g \in\left(G_{\mathbb{k}}\right)_{c}$, then $\Psi=\mu(g(c))=g(\mu(c))=g(\Psi)$, forcing $g \in Z_{G_{\mathrm{k}}}(\Psi)$. Thus, statement (iii) is an immediate consequence of statement (i).

Remark 4.2. If $\mathcal{D}_{M} \cong \mathcal{Q}\left(\mathbf{A}_{d}(\mathbb{C})\right)$, then $\bar{R}_{\mathbb{k}}$ is a prime ring by Corollary 4.2. So in this case we have that $\bar{R}_{\mathbb{k}}=\mathcal{R}$.

4.5. Recall from [2], [32], [1] that any prime PI-ring $\mathcal{A}$ has a simple Artinian ring of fractions $\mathcal{Q}(\mathcal{A})$ which satisfies the same identities as $\mathcal{A}$ and is spanned by $\mathcal{A}$ over its centre, $K$, which coincides with $\mathcal{Q}(Z(\mathcal{A}))$. Moreover, $\operatorname{dim}_{K} \mathcal{Q}(\mathcal{A})=d^{2}$, and after tensoring by a suitable algebraic field extension $\tilde{K}$ of $K$, the $\operatorname{ring} \mathcal{Q}(\mathcal{A})$ becomes the matrix algebra $\operatorname{Mat}_{d}(\tilde{K})$. Both $\mathcal{A}$ and $\mathcal{Q}(\mathcal{A})$ satisfy all the polynomial identities of $d \times d$ matrices over a commutative ring, but not those of smaller matrices, and $d$ can be characterized as the least positive integer such that $S_{2 d}\left(X_{1}, \ldots, X_{2 d}\right)=0$ for all $X_{1}, \ldots, X_{2 d} \in \mathcal{A}$, where

$$
S_{2 d}\left(X_{1}, \ldots, X_{2 d}\right):=\sum_{\sigma \in \mathfrak{S}_{2 d}}(\operatorname{sgn} \sigma) X_{\sigma(1)} \cdots X_{\sigma(2 d)} .
$$

Definition 4.1. The PI-degree of a prime PI-ring $\mathcal{A}$, denoted PI-deg $(\mathcal{A})$, is defined as the least positive integer $d$ such that $\mathcal{A}$ satisfies the standard identity $S_{2 d} \equiv 0$.

Definition 4.2. We say that $\mathcal{A}$ is an $A$ zumaya algebra over its centre $Z(\mathcal{A})$ if $\mathcal{A}$ is a finitely generated projective $Z(\mathcal{A})$-module and the natural map $\mathcal{A} \otimes_{Z(\mathcal{A})} \mathcal{A}^{\mathrm{op}} \rightarrow$ $\operatorname{End}_{Z(\mathcal{A})} \mathcal{A}$ is an isomorphism.

Now suppose that our PI-ring $\mathcal{A}$ is finitely generated over its centre $Z(\mathcal{A})$ which, in turn, is an affine algebra over $\mathbb{k}$. In this situation, it is known that $\operatorname{PI} \operatorname{deg}(\mathcal{A})=d(\mathcal{A})$, where $d(\mathcal{A})$ stands for the maximum $\mathbb{k}$-dimension of irreducible $\mathcal{A}$-modules; see [5], for example. Let $V$ be an irreducible $\mathcal{A}$-module, $P=\operatorname{Ann}_{\mathcal{A}} V$ and $\mathfrak{c}=P \cap Z(\mathcal{A})$, a maximal ideal of $Z(\mathcal{A})$. It follows from the Artin-Procesi theorem [22, Thm. 13.7.14] that the equality $\operatorname{dim} V=\mathrm{PI}-\operatorname{deg}(\mathcal{A})$ holds if and only if $\mathcal{A}_{\mathfrak{c}}=\mathcal{A} \otimes_{Z(\mathcal{A})} Z(\mathcal{A})_{\mathfrak{c}}$ is an Azumaya algebra over the local ring $Z(\mathcal{A})_{\mathfrak{c}}$; see [5] for more detail.

The Azumaya locus of $\mathcal{A}$, denoted $\operatorname{Az}(\mathcal{A})$, is defined as

$$
\operatorname{Az}(\mathcal{A}):=\left\{\mathfrak{c} \in \operatorname{Specm} Z(\mathcal{A}) \mid \mathcal{A}_{\mathfrak{c}} \text { is an Azumaya algebra }\right\} .
$$

The above discussion shows that $\operatorname{Az}(\mathcal{A})$ consists of all $\mathfrak{c} \in \operatorname{Specm} Z(\mathcal{A})$ with $\mathcal{A}_{\mathfrak{c}} / \mathfrak{c} \mathcal{A}_{\mathfrak{c}} \cong$ $\operatorname{Mat}_{d(\mathcal{A})}(\mathbb{k})$, whilst the $\operatorname{Artin}-$ Procesi theorem yields that $\operatorname{Az}(\mathcal{A})$ is a nonempty Zariski open subset of $\operatorname{Specm} Z(\mathcal{A})$; see [22, Thm. 13.7.14(iii)].

4.6. In this subsection, we will prove Theorems $\mathrm{A}$ and $\mathrm{B}$. First suppose that $\mathcal{D}_{M} \cong$ $\mathcal{Q}\left(\mathbf{A}_{d}(\mathbb{C})\right)$. Then Corollary 4.2 says that $\bar{R}_{\mathbb{k}}=\mathcal{R}$ is a prime ring. It follows from Proposition 4.3 that there exists a finite algebraic extension $\widetilde{K} \cong \mathbb{k}\left(X_{1}, \ldots, X_{2 d}\right)$ of the centre $K$ of $\mathcal{Q}(\mathcal{R})$ (identified with $\mathcal{Q}\left(Z_{d}(\mathbb{k})\right)$, the centre of $\mathcal{Q}\left(\mathbf{A}_{d}(\mathbb{k})\right)$ ) such that

$$
\mathcal{Q}(\mathcal{R}) \otimes_{K} \widetilde{K} \cong \operatorname{Mat}_{l^{\prime}}\left(\mathcal{Q}\left(\mathbf{A}_{d}(\mathbb{k})\right) \otimes_{K} \widetilde{K}\right) \cong \operatorname{Mat}_{l^{\prime} p^{d}}(\widetilde{K})
$$


As a result, $\mathrm{PI}-\operatorname{deg}(\mathcal{R})=l^{\prime} p^{d}$. On the other hand, since the Azumaya locus of $\mathcal{R}$ is $G_{\mathbb{k}^{\mathrm{k}}}$-stable and the dominant morphism $\mu: \operatorname{Specm} Z(\mathcal{R})=\mathcal{V}(\mathcal{R}) \rightarrow \operatorname{Specm} Z_{p}(\mathcal{R})$ from 4.4 is $G_{\mathbb{k}^{-}}$equivariant, it must be that $\Psi \in \mu(\mathrm{Az}(\mathcal{R}))$. But then $\mu^{-1}(\Psi) \cap \mathrm{Az}(\mathcal{R}) \neq \emptyset$. Applying Proposition 4.4 now yields $\mu^{-1}(\Psi) \subset \mathrm{Az}(\mathcal{R})$.

Let $\mathfrak{c}(\Psi)$ denote the annihilator in $Z(\mathcal{R})$ of the the irreducible $\mathcal{R}$-module $\widetilde{M}_{\mathbb{k}, \Psi}$ introduced in 2.6. Since $\mathfrak{c}(\Psi) \in \mu^{-1}(\Psi)$, the preceding remark shows that $\mathcal{R}_{\mathfrak{c}(\Psi)}$ is an Azumaya algebra. As $Z(\mathcal{R})_{\mathfrak{c}(\Psi)}$ is a local ring, our discussion in 4.5 now yields that

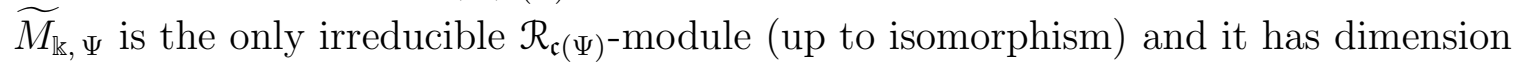
equal to $d(\mathcal{R})=$ PI- $\operatorname{deg}(\mathcal{R})$. Therefore,

$$
l^{\prime} p^{d}=\mathrm{PI}-\operatorname{deg}(\mathcal{R})=\operatorname{dim}_{\mathbb{k}} \widetilde{M}_{\mathbb{k}, \Psi}=l p^{d}=\left(\operatorname{dim}_{\mathbb{C}} M\right) p^{d} .
$$

Since $l^{\prime}=\operatorname{rk}\left(U(\mathfrak{g}) / I_{M}\right)$, Theorem B follows.

It remains to prove Theorem A. Applying Proposition 4.4 and arguing as before we obtain the inclusion $\mu^{-1}(\Psi) \subset \operatorname{Az}(\mathcal{R})$ and hence the equality $\operatorname{PI}-\operatorname{deg}(\mathcal{R})=l p^{d}$. On the other hand, Proposition 4.1 says that $\mathcal{Q}\left(\bar{R}_{\mathbb{k}}\right) \cong \mathfrak{C}_{\mathbb{k}} \otimes \mathfrak{D}_{\mathbb{k}}$, where $\mathfrak{D}_{\mathbb{k}}$ is the centraliser of $\mathfrak{C}_{\mathbb{k}} \cong \operatorname{Mat}_{l^{\prime}}(\mathbb{k})$ in $\mathcal{Q}\left(\bar{R}_{\mathbb{k}}\right)$. Since $\mathcal{Q}\left(\bar{R}_{\mathbb{k}}\right)$ is a semiprime Artinian ring, so is $\mathfrak{D}_{\mathbb{k}}$. Therefore, $\mathfrak{D}_{\mathbb{k}} \cong \bigoplus_{j=1}^{\nu^{\prime}} \mathfrak{D}_{\mathbb{k}, j}$ for some simple Artinian rings $\mathfrak{D}_{\mathbb{k}, j}$. But we know that $\mathcal{Q}\left(\bar{R}_{\mathbb{k}}\right)=\bigoplus_{j=1}^{\nu} \mathcal{Q}\left(\bar{R}_{\mathbb{k}} / \bar{I}_{j}\right)$ and each $\mathcal{Q}\left(\bar{R}_{\mathbb{k}} / \bar{I}_{j}\right)$ is a simple Artinian ring; see our discussion in 4.4. Since $\mathcal{Q}\left(\bar{R}_{\mathbb{k}}\right) \cong \bigoplus_{j=1}^{\nu^{\prime}}\left(\mathfrak{C}_{\mathbb{k}} \otimes \mathfrak{D}_{\mathbb{k}, j}\right)$ and each $\mathfrak{C}_{\mathbb{k}} \otimes \mathfrak{D}_{\mathbb{k}, j}$ is a simple Artinian ring, we now deduce that $\nu=\nu^{\prime}$ and

$$
\mathcal{Q}(\mathcal{R})=\mathcal{Q}\left(\bar{R}_{\mathbb{k}} / \bar{I}_{\ell}\right) \cong \mathfrak{C}_{\mathbb{k}} \otimes \mathfrak{D}_{\mathbb{k}, \ell^{\prime}}
$$

for some $\ell^{\prime} \leq \nu$. As $\mathfrak{C}_{\mathbb{k}} \cong \operatorname{Mat}_{l^{\prime}}(\mathbb{k})$, our discussion in 4.5 then shows that $l^{\prime}$ divides PI-deg $(\mathcal{Q}(\mathcal{R}))=\operatorname{PI}-\operatorname{deg}(\mathcal{R})=l p^{d}$. As $\Pi(A)$ contains almost all primes in $\mathbb{N}$, we can find $\mathfrak{P} \in \operatorname{Specm} A$ such that $l^{\prime}$ is coprime to $p=\operatorname{char} A / \mathfrak{P}$. Then we see that $l^{\prime}=\operatorname{rk}\left(U(\mathfrak{g}) / I_{M}\right)$ must divide $l=\operatorname{dim}_{\mathbb{C}} M$, which completes the proof of Theorem A.

Remark 4.3. Let $\mathfrak{g}=\mathfrak{s p}_{2 n}$ with $n \geq 3$ and let $e \in \mathfrak{g}$ be a nilpotent element corresponding to partition $\left(2^{n}\right)$ of $2 n$. We have already mentioned in the Introduction that $I_{\max }(\rho / 2)$ is a completely prime primitive ideal of $U(\mathfrak{g})$ with associated variety equal to $\overline{\mathcal{O}(e)}$. Hence $I_{\max }(\rho / 2)=I_{M}$ for some finite dimensional irreducible $U(\mathfrak{g}, e)$-module $M$. It is known (and not hard to see) that in the present case the action of ad $h$ on $\mathfrak{g}$ gives rise to a short $\mathbb{Z}$-grading

$$
\mathfrak{g}=\mathfrak{g}(-2) \oplus \mathfrak{g}(0) \oplus \mathfrak{g}(2), \quad \mathfrak{g}(i)=\{x \in \mathfrak{g} \mid[h, x]=i x\}
$$

such that $\mathfrak{g}(0) \cong \mathfrak{g l}(V)$ and $\mathfrak{g}(2) \cong S^{2}(V)$ as $\mathfrak{g}(0)$-modules (here $V$ is an $n$-dimensional vector space over $\mathbb{C}$ ). Furthermore, $e \in S^{2}(V)$ corresponds to a nondegenerate quadratic form on $V^{*}$ and the centraliser $\mathfrak{g}_{e}$ is a graded subspace of $\mathfrak{g}(0) \oplus \mathfrak{g}(2)$. Therefore, $\mathfrak{g}_{e}=\mathfrak{g}_{e}(0) \oplus \mathfrak{g}(2)$ and $\mathfrak{g}_{e}(0)=\mathfrak{g}_{e} \cap \mathfrak{g}(0)$ is isomorphic to $\mathfrak{s o}(V)$ as Lie algebras. Since $\operatorname{dim} V \geq 3$ and $V$ is an irreducible $\mathfrak{s o}(V)$-module, the Lie algebra $\mathfrak{s o}(V)$ is semisimple and the subspace of $\mathfrak{s o}(V)$-invariants in $S^{2}(V)$ is one-dimensional. This implies that $\mathfrak{g}_{e}=\left[\mathfrak{g}_{e}, \mathfrak{g}_{e}\right] \oplus \mathbb{C} e$. But then the results proved in [28, Sect. 2] show that the commutative quotient $U(\mathfrak{g}, e)^{\text {ab }}$ of $U(\mathfrak{g}, e)$ is generated by a Casimir element $\Omega$ of $U(\mathfrak{g})$ (one should keep in mind here that $\Omega$ can be regarded as one of the PBW generators of $U(\mathfrak{g}, e)$, namely, $\left.\Omega=\Theta_{e}\right)$. Since the Krull dimension of $U(\mathfrak{g}, e)^{\text {ab }}$ is positive by $[31$, Thm. 1.2] we now obtain that $U(\mathfrak{g}, e)^{\mathrm{ab}} \cong \mathbb{C}[X]$ as algebras. 
For $t \in \mathbb{C}$ we denote by $L_{t}$ the $\mathfrak{g}$-module induced from the one-dimensional module $\mathbb{C} v_{t}$ over the parabolic subalgebra $\mathfrak{g}(0) \oplus \mathfrak{g}(2)$ of $\mathfrak{g}$ such that $z \cdot v_{t}=t v_{t}$, where $z$ is a fixed nonzero central element of the Levi subalgebra $\mathfrak{g}(0) \cong \mathfrak{g l}(V)$ of $\mathfrak{g}$. By Conze's theorem, each ideal $I_{t}:=\operatorname{Ann}_{U(\mathfrak{g})} L_{t}$ of $U(\mathfrak{g})$ is completely prime (hence prime). As the centre of $U(\mathfrak{g})$ acts on $L_{t}$ by scalar operators, the Dixmier-Møglin equivalence yields that each $I_{t}$ is an induced primitive ideal of $U(\mathfrak{g})$. Note that in the present case $\mathfrak{m}=\mathfrak{g}(-2)$ and each $L_{t}$ is a free $U(\mathfrak{m})$-module of rank 1 . Therefore, the subspace $M_{t}:=\left\{\psi \in L_{t}^{*} \mid \psi\left(\mathfrak{m}_{\chi} \cdot L_{t}\right)=0\right\}$ of the (full) dual space $L_{t}^{*}$ is a one-dimensional $U(\mathfrak{g}, e)$-module (recall that $\mathfrak{m}_{\chi}$ is the subspace of $U(\mathfrak{g})$ spanned by all $x-\chi(x)$ with $x \in \mathfrak{m})$. We denote by $\widetilde{M}_{t}$ the submodule of the $\mathfrak{g}$-module $L_{t}^{*}$ generated by $M_{t}$. Since $\operatorname{Ann}_{U(\mathfrak{g})} V^{*}=\left(\operatorname{Ann}_{U(\mathfrak{g})} V\right)^{\top}$ for any $\mathfrak{g}$-module $V$, for every $t \in \mathbb{C}$ we have the inclusion $I_{t}^{\top} \subseteq \operatorname{Ann}_{U(\mathfrak{g})} \widetilde{M}_{t}$ (here $T$ stands for the principal anti-automorphism of $U(\mathfrak{g})$ ). The Dixmier-Moerlin equivalence implies that each $I_{t}^{\top}$ is a primitive ideal of $U(\mathfrak{g})$, whilst a routine verification shows that $\operatorname{gr}\left(I_{t}^{\top}\right)=\operatorname{gr}\left(I_{t}\right)$ and hence $\mathcal{V} \mathcal{A}\left(I_{t}^{\top}\right)=\mathcal{V} \mathcal{A}\left(I_{t}\right)$; see $[28,3.3]$ for more detail.

It is immediate from Skryabin's equivalence that $\widetilde{M}_{t} \cong Q_{e} \otimes_{U(\mathfrak{g}, e)} M_{t}$ as $\mathfrak{g}$-modules; see [34]. Then $\operatorname{Ann}_{U(\mathfrak{g})} \widetilde{M}_{t}=I_{M_{t}}$ and hence $\mathcal{V} \mathcal{A}\left(I_{t}^{\top}\right) \supseteq \mathcal{V} \mathcal{A}\left(I_{M_{t}}\right)$. Since each induced $\mathfrak{g}$-module $L_{t}$ is holonomic and $e$ is a Richardson element of $\mathfrak{g}(0) \oplus \mathfrak{g}(2)$, we also have that $\operatorname{dim} \mathcal{V} \mathcal{A}\left(I_{t}\right)=\operatorname{dim} \overline{\mathcal{O}(e)}=\operatorname{dim} \mathcal{V} \mathcal{A}\left(I_{M_{t}}\right)$; see [28, Thm. 3.1(ii)]. In view of the above remarks, this gives $\mathcal{V} \mathcal{A}\left(I_{t}^{\top}\right)=\mathcal{V} \mathcal{A}\left(I_{t}\right)=\mathcal{V} \mathcal{A}\left(I_{M_{t}}\right)$. As both $I_{t}^{\top}$ and $I_{M_{t}}$ are primitive ideals of $U(\mathfrak{g})$, applying [3, Corollar 3.6] yields $I_{t}^{\top}=I_{M_{t}}$. Since for $\mathfrak{g}=\mathfrak{s p}_{2 n}$ all homogeneous ( $\operatorname{Ad} G$ )-invariants of $S(\mathfrak{g})$ have even degrees, one observes easily by using the symmetrisation map $U(\mathfrak{g}) \stackrel{\sim}{\rightarrow} S(\mathfrak{g})$ that every set $\chi_{\lambda}$ is stable under the principal anti-automorphism $T$. The uniqueness of $I_{\max }(\rho / 2) \in X_{\rho / 2}$ then yields $I_{\max }(\rho / 2)=I_{\max }(\rho / 2)^{\top}$.

On the other hand, it is well known that there exists a quadratic polynomial $\phi \in$ $\mathbb{C}[X]$ such that the eigenvalue of $\Omega$ on the parabolically induced $\mathfrak{g}$-module $L_{t}$ equals $\phi(t)$ for any $t \in \mathbb{C}$. In conjunction with the above this shows that for every $\varkappa \in \mathbb{C}$ there is a $t(\varkappa) \in \mathbb{C}$ such that $\Omega$ acts on $\widetilde{M}_{t(\varkappa)}$ as $\varkappa$ Id. Since $U(\mathfrak{g}, e)^{\text {ab }}=\mathbb{C}[\Omega]$ by our earlier remarks, we now deduce that all primitive ideals $I_{N}$ associated with onedimensional $U(\mathfrak{g}, e)$-modules $N$ belong to the set $\left\{I_{t}^{\top} \mid t \in \mathbb{C}\right\}$. Now $I_{\max }(\rho / 2)=$ $I_{M}$ for some finite dimensional irreducible $U(\mathfrak{g}, e)$-module $M$. If $\operatorname{dim} M=1$, then $I_{\max }(\rho / 2)^{\top}=I_{\max }(\rho / 2)=I_{t_{0}}^{\top}$ for some $t_{0} \in \mathbb{C}$. But then $I_{\max }(\rho / 2)=I_{t_{0}}$. Since the primitive ideal $I_{\max }(\rho / 2)=I_{M}$ is not induced by [24, p. 45], we reach a contradiction thereby proving that $\operatorname{dim} M>1$. As $\operatorname{rk}\left(U(\mathfrak{g}) / I_{\max }(\rho / 2)\right)=1$, Theorem B implies that the Goldie field of the primitive quotient $U(\mathfrak{g}) / I_{\max }(\rho / 2)$ is not isomorphic to a Weyl skew-field. This example shows that Joseph's version of the Gelfand-Kirillov conjecture fails for $\mathfrak{g}=\mathfrak{s p}_{2 n}$ with $n \geq 3$.

Remark 4.4. It seems that any attempt to generalise Theorem B would require a rather detailed information on the structure of the Goldie field $\mathcal{D}_{M}$. In the proof given above it was crucial for us to know that $\mathcal{D}_{M}$ is generated as a skew-field over $\mathbb{C}$ by its $A$-subalgebra $\Delta$ with the following property: for every $p \in \Pi(A)$ the $\mathbb{k}$-algebra $\Delta_{\mathbb{k}}:=\Delta \otimes_{A} \mathbb{k}$ becomes the full matrix algebra $\operatorname{Mat}_{p^{d}}(\widetilde{K})$ after a suitable algebraic field extension $\widetilde{K} / Q\left(Z\left(\Delta_{\mathbb{k}}\right)\right)$ of the fraction field of the centre of $\Delta_{\mathbb{k}}$. When $\mathcal{D}_{M}$ is a Weyl 
skew-field, this condition is satisfied with the obvious choice of $\Delta$, but the validity of Conjecture 4.1 alone would not guarantee that $\mathcal{D}_{M}$ contains an $A$-subalgebra $\Delta$ as above with the property that PI- $\operatorname{deg}\left(\Delta_{\mathbb{k}}\right)=p^{d}$. The best one could hope for would be an equality PI-deg $\left(\Delta_{\mathbb{k}}\right)=s p^{d}$ with an explicit bound on $s$ independent of $p$. One might, for example, wonder whether $s$ always divides the order of the Weyl group $W=\left\langle s_{\gamma} \mid \gamma \in \Phi\right\rangle$ or, more strongly, whether $\mathcal{D}_{M}$ is always a crossed product of a Weyl skew-field $\mathcal{F}$ and a finite group acting on $\mathcal{F}$ by algebra automorphisms. Such unexplored possibilities make Conjecture 4.1 more flexible and hence harder to disprove than the Gelfand-Kirillov conjecture for primitive quotients.

4.7. Let $M$ and $M^{\prime}$ be two generalised Gelfand-Graev models of a primitive ideal $\mathcal{J} \in X_{\mathcal{O}}$, so that $\mathcal{J}=I_{M}=I_{M^{\prime}}$. As we already mentioned in the Introduction, it was conjectured by the author and proved by Losev in $[20]$ that $\left[M^{\prime}\right]={ }^{\gamma}[M]$ for some $\gamma \in \Gamma(e)$. We would like to conclude this paper by showing that Conjecture 4.1 implies Losev's result.

Suppose $\bar{R}_{\mathbb{k}}$ is a prime ring and let $l=\operatorname{dim} V, l^{\prime}=\operatorname{dim} V^{\prime}$. Let $\Gamma$ be a subset of $C(e)=G_{e} \cap G_{f}$ which maps bijectively onto $\Gamma(e)$ under the canonical homomorphisms $C(e) \rightarrow \Gamma(e)=C(e) / C(e)^{\circ}$. Let us assume for a contradiction that $M^{\prime} \nsucceq{ }^{\gamma} M$ for any $\gamma \in \Gamma$. Arguing as in 2.6 we can find an admissible ring $A \subset \mathbb{C}$ and free $A$ submodules $M_{A}$ and $M_{A}^{\prime}$ of $M$ and $M^{\prime}$, respectively, stable under $U\left(\mathfrak{g}_{A}, e\right)$ and such that $M \cong M_{A} \otimes_{A} \mathbb{C}$ and $M^{\prime} \cong M_{A}^{\prime} \otimes_{A} \mathbb{C}$. For every $p \in \Pi(A)$ we then get $U\left(\mathfrak{g}_{\mathbb{k}}, e\right)$ modules $M_{\mathbb{k}}=M_{A} \otimes_{A} \mathbb{k}$ and $M_{\mathbb{k}}^{\prime}=M_{A}^{\prime} \otimes_{A} \mathbb{k}$, where $\mathbb{k}=\overline{\mathbb{F}}_{p}$. As in 2.6 we localise further to reduce to the case where $M_{\mathbb{k}}$ and $M_{\mathbb{k}}^{\prime}$ are irreducible $U\left(\mathfrak{g}_{\mathbb{k}}, e\right)$-modules for all $p \in \Pi(A)$. Associated with $M_{\mathbb{k}}$ and $M_{\mathbb{k}}^{\prime}$ are $\bar{R}_{\mathbb{k}}$-modules $\widetilde{M}_{\mathbb{k}, \Psi}$ and $\widetilde{M}_{\mathbb{k}, \Psi^{\prime}}^{\prime}$, where $\Psi, \Psi^{\prime} \in \chi+\mathfrak{m}_{\mathbb{k}}^{\perp}$; see 2.6 for more detail.

Recall from 2.3 that $U\left(\mathfrak{g}_{A}, e\right)$ is a free $A$-module with basis consisting of the PBW monomials in $\Theta_{1}, \ldots, \Theta_{r}$. Since $\Gamma$ is a finite set, we may assume (after extending $A$ if necessary) that the $A$-form $U\left(\mathfrak{g}_{A}, e\right)$ of $U(\mathfrak{g}, e)$ is stable under the action of the subgroup of $C(e)$ generated by $\Gamma$. Then each ${ }^{\gamma} M_{A}$ with $\gamma \in \Gamma$ can be regarded as a $U\left(\mathfrak{g}_{A}, e\right)$-module. For $\gamma \in \Gamma$, the equality $\operatorname{Hom}_{U(\mathfrak{g}, e)}\left({ }^{\gamma} M, M^{\prime}\right)=0$ comes down to the fact that a certain homogeneous system of linear equations in $l l^{\prime}$ unknowns with coefficients in $A$ has no nonzero solutions. After inverting in $A$ one of the nonzero $l l^{\prime} \times l l^{\prime}$ minors of the matrix of this homogeneous system we may assume that $\operatorname{Hom}_{U\left(\mathfrak{g}_{\mathbb{k}}, e\right)}\left({ }^{\gamma} M_{\mathbb{k}}, M_{\mathbb{k}}^{\prime}\right)=0$ for all $p \in \Pi(A)$ and all $\gamma \in \Gamma$.

Recall from [29] and [31] the subset $\pi(A)$ of $\Pi(A)$; it consists of all primes $p \in \mathbb{N}$ such that $A / \mathfrak{P} \cong \mathbb{F}_{p}$ for some $\mathfrak{P} \in \operatorname{Specm} A$. By [31, Lemma 4.4], the set $\pi(A)$ is infinite. The preceding remark then shows that no generality will be lost by assuming that $p \in \pi(A)$ and ${ }^{\gamma} M_{\mathbb{k}} \approx M_{\mathbb{k}}^{\prime}$ as $U\left(\mathfrak{g}_{\mathbb{k}}, e\right)$-modules for all $\gamma \in \Gamma$. Enlarging $A$ further if need be we may also assume that $\mathcal{J}=\operatorname{Ann}_{U(\mathfrak{g})} L(\lambda)$ and $\mathcal{J}_{A} \subseteq \operatorname{Ann}_{U\left(\mathfrak{g}_{A}\right)} L_{A}(\lambda)$ for some irreducible highest weight module $L(\lambda)$ and that $A$ satisfies all the requirements of [31, Sect. 4]. Since $\pi(A)$ is an infinite set, we may also assume that the base change $A \rightarrow A / \mathfrak{P} \hookrightarrow \mathbb{k}$ identifies $\Gamma \subset G(A)$ with a subset of $Z_{G_{\mathrm{k}}}(\chi)$ which maps onto the component group of $Z_{G_{\mathrm{k}}}(\chi)$ under the canonical homomorphism $Z_{G_{\mathrm{k}}}(\chi) \rightarrow$ $Z_{G_{\mathbb{k}}}(\chi) / Z_{G_{\mathbb{k}}}(\chi)^{\circ}$.

Let $\mathfrak{P} \in \operatorname{Specm} A$ be such that $A / \mathfrak{P} \cong \mathbb{F}_{p}$. As explained in $[31,4.5]$ the $R_{\mathbb{k}^{\mathrm{k}}}$-module $L_{\mathfrak{P}}(\lambda)=L_{A}(\lambda) \otimes_{A} \mathbb{k}_{\mathfrak{P}}$ has an irreducible quotient, $L_{\mathfrak{P}}^{\eta}(\lambda)$, which has $p$-character 
$\eta \in\left(\operatorname{Ad}^{*} G_{\mathbb{k}}\right) \chi$. As the ideal $N_{\mathrm{k}}$ is nilpotent, $L_{\mathfrak{P}}^{\eta}(\lambda)$ is an irreducible $\bar{R}_{\mathrm{k}}$-module. Since we assume that the algebra $\bar{R}_{\mathbb{k}}$ is prime, the variety $\operatorname{Specm} Z_{p}\left(\bar{R}_{\mathrm{k}}\right) \subset \mathfrak{g}_{\mathbb{k}}^{*}$ is irreducible and $\left(\mathrm{Ad}^{*} G_{\mathbb{k}}\right)$-stable. By Proposition 4.2 , it has dimension $2 d$ which forces Specm $Z_{p}\left(\bar{R}_{\mathbb{k}}\right)=\overline{\left(\operatorname{Ad}^{*} G_{\mathbb{k}}\right) \chi}$. But then both $\Psi$ and $\Psi^{\prime}$ are $\left(\operatorname{Ad}^{*} G_{\mathbb{k}}\right)$-conjugate to $\chi$. As explained in Remark 2.1 we can replace $\Psi$ and $\Psi^{\prime}$ by their $\left(\operatorname{Ad}^{*} \mathcal{M}_{\mathbb{k}}\right)$-conjugates. In view of [31, Lemma 3.2] and standard properties of Slodowy slices, we therefore may assume further that $\Psi=\Psi^{\prime}=\chi$.

Denote by $\mathfrak{c}$ and $\mathfrak{c}^{\prime}$ the annihilators in $Z\left(\bar{R}_{\mathrm{k}}\right)$ of $\widetilde{M}_{\mathrm{k}, \Psi}$ and $\widetilde{M}_{\mathrm{k}, \Psi^{\prime}}^{\prime}$, respectively. As $\mu(\mathfrak{c})=\mu\left(\mathfrak{c}^{\prime}\right)=\chi$, Proposition 4.4 shows that $\mathfrak{c}^{\prime}=\gamma_{0}(\mathfrak{c})$ for some $\gamma_{0} \in \Gamma$. On the other hand, arguing as in 4.6 it is straightforward to see that $\mathfrak{c}, \mathfrak{c}^{\prime} \in \operatorname{Az}\left(\bar{R}_{\mathbb{k}}\right)$. From this it follows that $\widetilde{M}_{\mathrm{k}, \Psi^{\prime}}^{\prime} \cong \gamma_{0}\left(\widetilde{M}_{\mathbb{k}, \Psi}\right)$ as $\bar{R}_{\mathbb{k}}$-modules and hence as $U_{\chi}\left(\mathfrak{g}_{\mathbb{k}}\right)$-modules. In view of the Morita equivalence mentioned in 2.5 this implies that

$$
M_{\mathrm{k}}^{\prime} \cong \mathrm{Wh}_{\chi}\left(\widetilde{M}_{\mathrm{k}, \Psi^{\prime}}^{\prime}\right) \cong \mathrm{Wh}_{\chi} \gamma_{0}\left(\widetilde{M}_{\mathbb{k}, \Psi}\right) \cong \mathrm{Wh}_{\chi}^{\gamma_{0}}\left(Q_{e}^{\chi} \otimes_{U_{\chi}\left(\mathrm{g}_{\mathrm{k}}, e\right)} M_{\mathbb{k}}\right)
$$

as $U\left(\mathfrak{g}_{\mathfrak{k}}, e\right)$-modules. The adjoint action of $Z_{G_{\mathrm{k}}}(\chi)$ on $\mathfrak{g}_{\mathrm{k}}$ gives rise to a natural group homomorphism $Z_{G_{\mathrm{k}}}(\chi) \rightarrow$ Aut $U_{\chi}\left(\mathfrak{g}_{\mathrm{k}}\right)$. By [27, Thm. 2.3(i)], the left regular module $U_{\chi}\left(\mathfrak{g}_{\mathrm{k}}\right)$ is isomorphic to a direct sum of $p^{d}$ copies of $Q_{e}^{\chi}$. Since the same is true with $\gamma_{0}\left(Q_{e}^{\chi}\right) \subset U_{\chi}\left(\mathfrak{g}_{\mathrm{k}}\right)$ in place of $Q_{e}^{\chi}$, we see that the projective $U_{\chi}\left(\mathfrak{g}_{\mathrm{k}}\right)$-modules $Q_{\chi}^{e}$ and $\gamma_{0}\left(Q_{e}^{\chi}\right) \cong \gamma_{0}\left(Q_{\chi}^{e}\right)$ are isomorphic. Note that the right action of $U_{\chi}\left(\mathfrak{g}_{\mathrm{k}}, e\right)$ on ${ }^{\gamma_{0}}\left(Q_{e}^{\chi}\right)$ is the $\gamma_{0}$-twist of that on $Q_{e}^{\chi}$, where $\gamma_{0} \in \Gamma$ is now regarded as an automorphism of $U\left(\mathfrak{g}_{\mathrm{k}}, e\right)$. Comparing common $\left(\operatorname{Ad} \gamma_{0}\right)\left(\mathfrak{m}_{\mathrm{k}}\right)$-eigenvectors one observes that the $U_{\chi}\left(\mathfrak{g}_{\mathrm{k}}\right)$ modules ${ }^{\gamma_{0}}\left(\widetilde{M}_{\mathbb{k}, \Psi}\right)$ and ${ }^{\gamma_{0}}\left(Q_{e}^{\chi}\right) \otimes_{U_{\chi}\left(\mathfrak{g}_{\mathrm{k}}, e\right)}{ }^{\gamma_{0}} M_{\mathbb{k}}$ are isomorphic. As a consequence,

$$
\gamma_{0}\left(\widetilde{M}_{\mathbb{k}, \Psi}\right) \cong \gamma^{\gamma_{0}}\left(Q_{e}^{\chi}\right) \otimes_{U_{\chi}\left(\mathfrak{g}_{\mathfrak{k}}, e\right)}{ }^{\gamma_{0}} M_{\mathbb{k}} \cong Q_{e}^{\chi} \otimes_{U_{\chi}\left(\mathfrak{g}_{\mathfrak{k}}, e\right)}{ }^{\gamma_{0}} M_{\mathbb{k}}
$$

as $U_{\chi}\left(\mathfrak{g}_{\mathfrak{k}}\right)$-modules. In view of the above-mentioned Morita equivalence this entails that $M_{\mathbb{k}} \cong{ }^{0} M_{\mathbb{k}}$ as $U\left(\mathfrak{g}_{\mathbb{k}}, e\right)$-modules.

We have reached a contradiction thereby showing that $M^{\prime} \cong{ }^{\gamma} M$ for some $\gamma \in \Gamma$.

\section{REFERENCES}

[1] E.P. Armendariz and C.R. Hajarnavis, A note on semiprime rings algebraic over their centres, Comm. Algebra 17 no. 7 (1989) 1627-1631.

[2] G.M. Bergman and L.W. Small, P.I. degrees and prime ideals, J. Algebra 33 (1975) 435-462.

[3] W. Borho and H. Kraft, Über die Gelfand-Kirillov-Dimension, Math. Ann. 220 (1976) 1-24.

[4] N. Bourbaki, Algèbre Commutative, Hermann, Paris, 1961 (Ch. I/II), 1962 (Ch. III/IV), 1964 (Ch. V/VI), 1965 (Ch. VII).

[5] K.A. Brown and K.R. Goodearl, Homological aspects of Noetherian PI Hopf algebras and irreducible modules of maximal dimension, J. Algebra 198 (1997) 240-265.

[6] J. Brundan, Moglin's theorem and Goldie rank polynomials in Cartn type A, preprint, 2010, available at arXiv:math.RT/1010.0640v1.

[7] J. Brundan and A. Kleshchev, Shifted Yangians and finite W-algebras, Adv. Math. 200 (2006) 136-195.

[8] J. Brundan and A. Kleshchev, Representations of shifted Yangians and finite $W$-algebras, Mem. Amer. Math. Soc. 196 (2008) no. 918.

[9] J. Dixmier, Algèbres Enveloppantes, Gauthier-Villars, Paris, Bruxelles, Montréal, 1974.

[10] D. Eisenbud, Commutative Algebra with a View Towards Algebraic Geometry, Graduate Texts in Mathematics, Vol. 150, Springer, New York, Berlin, etc., 1994.

[11] W.L. Gan and V. Ginzburg, Quantization of Slodowy slices, Int. Math. Res. Not. 5 (2002) $243-255$. 
[12] I.M. Gelfand and A.A. Kirillov, Sur les corps liés aux algèbres enveloppantes des algèbres de Lie, Inst. Hautes Études Sci. Publ. Math. 31 (1966) 5-19.

[13] V. Ginzburg, Harish-Chandra bimodules for quantized Slodowy slices, Represent. Theory 13 (2009) 236-371.

[14] A. Goldie, The structure of Noetherian rings, In "Lectures on Rings and Modules (Tulane Univ. Ring and Operator Theory Year 1970-1971, I", Lecture Notes in Mathematics, Vol. 246, Springer-Verlag, Berlin, Heidelberg, 1972, pp. 213-321.

[15] J.C. Jantzen, Einhülende Algebren Halbeinfacher Lie-Algebren, Ergebnisse der Math., Vol. 3, Springer, New York, Tokyo, etc., 1983.

[16] A. Joseph, On the Gelfand-Kirillov conjecture for induced ideals in the semisimple case, Bull. Soc. Math. France 107 (1979) 139-159.

[17] A. Joseph, Kostant's problem, Goldie rank and the Gelfand-Kirillov conjecture, Invent. Math. 56 (1980) 191-213.

[18] B. Kostant, On Whittaker vectors and representation theory, Invent. Math. 48 (1978) 101-184.

[19] I.V. Losev, Quantized symplectic actions and W-algebras, J. Amer. Math. Soc. 23 (2010) 35-59.

[20] I.V. Losev, Finite dimensional representations of $W$-algebras, preprint, 2008, available at arXiv: math.RT/0807.1023v6.

[21] I.V. Losev, On the structure of the category $\mathcal{O}$ for $W$-algebras, preprint, 2008, available at arXiv:math.RT/0812.1584v3.

[22] J.C. McConnell and J.C. Robson, Noncommutative Noetherian Rings, Pure and Appl. Math, Whiley-Interscience, Chichester, New York, etc, 1988.

[23] W.M. McGovern, Rings of regular functions on nilpotent orbits. II. Model algebras and orbits, Comm. Algebra 22 no. 3 (1994) 765-772.

[24] W.M. McGovern, Completely prime maximal ideals and quantization, Mem. Amer. Math. Soc. 108 (1994) no. 519.

[25] C. Mœglin, Idéaux primitifs complètement premiers de l'algèbre enveloppante de $\mathfrak{g l}(n, \mathbb{C})$, J. Algebra 106 (1987) 287-366.

[26] A. Premet, Irreducible representations of Lie algebras of reductive groups and the Kac-Weisfeiler conjecture, Invent. Math. 121 (1995) 79-117.

[27] A. Premet, Special transverse slices and their enveloping algebras, Adv. Math. 170 (2002) 1-55.

[28] A. Premet, Enveloping algebras of Slodowy slices and the Joseph ideal, J. Eur. Math. Soc. 9 (2007) 487-543.

[29] A. Premet, Primitive ideals, non-restricted representations and finite $W$-algebras, Mosc. Math. J. 7 (2007) 743-762.

[30] A. Premet, Modular Lie algebras and the Gelfand-Kirillov conjecture, Invent. Math. 181 (2010) $395-420$.

[31] A. Premet, Commutative quotients of finite W-algebras, Adv. Math. 225 (2010) 269-306.

[32] L.H. Rowen, Maximal quotients of semiprime PI-algebras, Trans. Amer. Math. Soc. 196 (1974) 127-135.

[33] W. Schelter, Non-commutative P.I. rings are catenary, J. Algebra 51 (1978) 12-18.

[34] S. Skryabin, A category equivalence, Appendix to [27].

School of Mathematics, University of Manchester, Oxford Road, M13 9PL, UK

E-mail address: Alexander.Premet@manchester.ac.uk 